\title{
Highly rotating fluids with vertical stratification for periodic data and vanishing vertical viscosity
}

\author{
Stefano Scrobogna*
}

July 27, 2017

\begin{abstract}
We prove that the three-dimensional, periodic primitive equations with zero vertical diffusivity are globally well posed if the Rossby and Froude number are sufficiently small. The initial data is considered to be of zero horizontal average and the space domain may be resonant. No smallness assumption is assumed on the initial data.
\end{abstract}

\section{Introduction}

The primitive equations describe the hydro-dynamical flow in a large scale (of order of hundreds or thousands of kilometers) on the Earth, typically the ocean or the atmosphere, under the assumption that the vertical motion is much smaller than the horizontal one and that the fluid layer depth is small compared to the radius of the Earth. Concerning the difference between horizontal and vertical scale, it is observed that for geophysical fluids the vertical component of the diffusion term (viscosity or thermal diffusivity in the case of primitive equations) is much smaller than the horizontal components. In the case of rotating fluids between two planes (see 27 for the first work in which the initial data is well prepared, in the sense that it is a two-dimensional vector field and [34 and [17] for the generic case) the viscosity assumes the form $\left(-v_{h} \Delta_{h}-\varepsilon \beta \partial_{3}^{2}\right)$, with $\Delta_{h}=\partial_{1}^{2}+\partial_{2}^{2}$, whence such geophysical motivation justifies the study of anisotropic (i.e. non-spherically symmetric) viscosities.

The primitive system consists in the following equations

$$
\left\{\begin{array}{l}
\partial_{t} v^{1, \varepsilon}+v^{\varepsilon} \cdot \nabla v^{1, \varepsilon}-v_{h} \Delta_{h} \nu^{1, \varepsilon}-v_{\nu} \partial_{3}^{2} v^{1, \varepsilon}-\frac{1}{\varepsilon} v^{2, \varepsilon}=-\frac{1}{\varepsilon} \partial_{1} \Phi_{\varepsilon}+f_{1} \\
\partial_{t} v^{2, \varepsilon}+v^{\varepsilon} \cdot \nabla v^{2, \varepsilon}-v_{h} \Delta_{h} v^{2, \varepsilon}-v_{\nu} \partial_{3}^{2} v^{2, \varepsilon}+\frac{1}{\varepsilon} v^{1, \varepsilon}=-\frac{1}{\varepsilon} \partial_{2} \Phi_{\varepsilon}+f_{2} \\
\partial_{t} v^{3, \varepsilon}+v^{\varepsilon} \cdot \nabla v^{3, \varepsilon}-v_{h} \Delta_{h} v^{3, \varepsilon}-v_{\nu} \partial_{3}^{2} v^{2, \varepsilon}+\frac{1}{F \varepsilon} T^{\varepsilon}=-\frac{1}{\varepsilon} \partial_{3} \Phi_{\varepsilon}+f_{3} \\
\partial_{t} T^{\varepsilon}+v^{\varepsilon} \cdot \nabla T^{\varepsilon}-v_{h}^{\prime} \Delta_{h} T^{\varepsilon}-v_{\nu}^{\prime} \partial_{3}^{2} T^{\varepsilon}-\frac{1}{F \varepsilon} v^{3, \varepsilon}=f_{4} \\
\operatorname{div} v^{\varepsilon}=0 \\
\left.\left(v^{\varepsilon}, T^{\varepsilon}\right)\right|_{t=0}=\left(v_{0}, T_{0}\right)=V_{0},
\end{array}\right.
$$

in the unknown $v^{\varepsilon}=\left(v^{1, \varepsilon}, v^{2 \varepsilon}, v^{3, \varepsilon}\right), T^{\varepsilon}, \Phi_{\varepsilon}$. In the following we write $V^{\varepsilon}=\left(v^{\varepsilon}, T^{\varepsilon}\right)=\left(V^{1, \varepsilon}, V^{2, \varepsilon}, V^{3, \varepsilon}, V^{4, \varepsilon}\right)$. All the functions described depend on a couple $(x, t) \in \mathbb{T}^{3} \times \mathbb{R}_{+}$where $\mathbb{T}^{3}$ represents the torus

$$
\mathbb{T}^{3}=\mathbb{R}^{3} / \prod_{i=1}^{3} a_{i} \mathbb{Z}=\prod_{i=1}^{3}\left[0,2 \pi a_{i}\right) .
$$

The only assumption which is made on the vertical viscosity is $v_{\nu}, v_{\nu}^{\prime} \geqslant 0$, while the horizontal viscosities $v_{h}, v_{h}^{\prime}$ are strictly positive constants. The results obtained will be uniform with respect to the vertical viscosities $\left(v_{v}, v_{v}^{\prime}\right)$ and hence from now on we can suppose them zero without loss of generality. We refer to [36] for a result of well-posedness of the Navier-Stokes equation in critical spaces in the whole space with anisotropic viscosity and to [37] for the periodic case.

Under the assumption $v_{\nu}=v_{\nu}^{\prime}=0$ we can rewrite the system $\overline{\mathrm{PE}_{\varepsilon}}$ in the more compact form

$$
\left\{\begin{array}{l}
\partial_{t} V^{\varepsilon}+v^{\varepsilon} \cdot \nabla V^{\varepsilon}-\mathbf{D} V^{\varepsilon}+\frac{1}{\varepsilon} \mathcal{A} V^{\varepsilon}=\frac{1}{\varepsilon}\left(-\nabla \Phi_{\varepsilon}, 0\right)+f \\
\operatorname{div} v^{\varepsilon}=0 \\
\left.V^{\varepsilon}\right|_{t=0}=V_{0}
\end{array}\right.
$$

\footnotetext{
${ }^{*}$ This research was partially supported by the Basque Government through the BERC 2014-2017 program and by the Spanish Ministry of Economy and Competitiveness MINECO: BCAM Severo Ochoa accreditation SEV-2013-0323.
} 
where

$$
\mathbf{D}=\left(\begin{array}{cccc}
v_{h} \Delta_{h} & 0 & 0 & 0 \\
0 & v_{h} \Delta_{h} & 0 & 0 \\
0 & 0 & v_{h} \Delta_{h} & 0 \\
0 & 0 & 0 & v_{h}^{\prime} \Delta_{h}
\end{array}\right) \quad \mathcal{A}=\left(\begin{array}{cccc}
0 & -1 & 0 & 0 \\
1 & 0 & 0 & 0 \\
0 & 0 & 0 & F^{-1} \\
0 & 0 & -F^{-1} & 0
\end{array}\right)
$$

with $v_{h}, v_{h}^{\prime}>0$ and $V^{\varepsilon}=\left(v^{\varepsilon}, T^{\varepsilon}\right)$.

This system is obtained by combining the effects of the Coriolis force and the vertical stratification induced by the Boussinesq approximation. We refer to [18], [38] or [20] for a discussion on the model and its derivations.

In the study of hydrodynamical flows on this scale two important phenomena have to be taken in consideration: the Earth rotation and the vertical stratification induced by the gravity. The Coriolis force induces a vertical rigidity on the fluid. Namely, in the asymptotic regime, the high rotation tends to stabilize the motion, which becomes constant in the direction parallel to the rotation axis: the fluid moves along vertical columns (the so called Taylor-Proudman columns), and the flow is purely horizontal.

Gravity forces the fluid masses to have a vertical structure: heavier layers lay under lighter ones. Internal movements of the fluid tend to destroy this structure and gravity tries to restore it, which gives a horizontal rigidity (to be opposed to the vertical rigidity induced by the rotation). In order to formally estimate the importance of this rigidity we also compare the typical time scale of the system with the Brunt-Väisälä frequency and introduce the Froude number $\varepsilon F$. We shall not give more details in here, we refer to [38], [18], [20].

The primitive equations are obtained with moment, energy and mass conservation (see $[22 \mid$ ). The coefficient $\varepsilon>0$ denotes the Rossby number, which is defined as

$$
\varepsilon=\frac{\text { displacement due to inertial forces }}{\text { displacement due to Coriolis force }} \text {. }
$$

As the characteristic displacement of a particle in the ocean within a day is very small compared to the displacement caused by the rotation of the Earth (generally $\varepsilon$ is of order $10^{-3}$ outside persistent currents such as the gulf stream), the Rossby number is supposed to be very small hence it is reasonable to study the behavior of the solutions to $\left(\mathrm{PE}_{\varepsilon}\right]$ in the limit regime as $\varepsilon \rightarrow 0$.

We denote the Froude number as $\varepsilon F$. Assuming that the Brunt-Väisälä frequency is constant, in the whole space $\mathbb{R}^{3}$, when $\varepsilon \rightarrow 0$, the formal limit of the system $\left[\mathrm{PE}_{\varepsilon}\right]$, when the viscosity is isotropic, is the quasi-geostrophic system

$$
\left\{\begin{array}{l}
\partial_{t} V_{\mathrm{QG}}+\Gamma(D) V_{\mathrm{QG}}=-\left(\begin{array}{c}
\nabla_{h}^{\perp} \\
0 \\
-F \partial_{3}
\end{array}\right) \Delta_{F}^{-1}\left(v_{\mathrm{QG}}^{h} \cdot \nabla_{h} \Omega\right), \\
\operatorname{div} v_{\mathrm{QG}}=0 \\
\left.V_{\mathrm{QG}}\right|_{t=0}=V_{\mathrm{QG}, 0},
\end{array}\right.
$$

and $\Gamma(D)$ is the pseudo-differential operator given by the formula

$$
\Gamma(D) u=\mathcal{F}^{-1}\left(\frac{|\xi|^{2}\left(v\left|\xi_{h}\right|^{2}+v^{\prime} F^{2} \xi_{3}^{2}\right)}{\left|\xi_{h}\right|^{2}+F^{2} \xi_{3}^{2}} \hat{u}(\xi)\right) .
$$

The differential operator $\Delta_{F}$ is defined as $\Delta_{F}=\partial_{1}^{2}+\partial_{2}^{2}+F^{2} \partial_{3}^{2}$, while its inverse $\Delta_{F}^{-1}$ in $L^{2}$ is the Fourier multiplier

$$
-\Delta_{F}^{-1} f=\mathcal{F}^{-1}\left(\frac{1}{\xi_{1}^{2}+\xi_{2}^{2}+F^{2} \xi_{3}^{2}} \hat{f}\right)
$$

The quantities $V_{\mathrm{QG}}$ and $\Omega$ are respectively called the quasi-geostrophic flow and the potential vorticity. We focus on the latter first, the potential vorticity is defined as

$$
\Omega=-\partial_{2} V_{\mathrm{QG}}^{1}+\partial_{1} V_{\mathrm{QG}}^{2}-F \partial_{3} V_{\mathrm{QG}}^{4},
$$

and it is related to the quasi-geostrophic flow via the 2D-like Biot -Savart law

$$
V_{\mathrm{QG}}=\left(\begin{array}{c}
-\partial_{2} \\
\partial_{1} \\
0 \\
-F \partial_{3}
\end{array}\right) \Delta_{F}^{-1} \Omega
$$


The vectors $v_{\mathrm{QG}}^{h}$ and $v_{\mathrm{QG}}$ represent respectively the first two and three components of the vector field $V_{\mathrm{QG}}$. In the present setting, i.e. with periodic data, the limit system is more involved than the one mentioned above. In this case, as well as in many problems with singular perturbation, the idea is to decompose the unknown (in the case of the system $\left(\overline{\mathrm{PE}_{\varepsilon}}\right)$ is $V^{\varepsilon}$ ) into two parts $V^{\varepsilon}=V_{\mathrm{ker}}^{\varepsilon}+V_{\mathrm{osc}}^{\varepsilon}$, where $V_{\mathrm{ker}}^{\varepsilon}$ belongs to the kernel of the perturbation $\mathbb{P} \mathcal{A}$, where $\mathbb{P}$ is the Leray projector in the first three components which leaves untouched the fourth one, and $V_{\text {osc }}^{\varepsilon}$ to its orthogonal complement. In the whole space it can be proved that the oscillating part, $V_{\text {osc }}^{\varepsilon}$, tends to zero strongly as $\varepsilon \rightarrow 0$. In the case of periodic data instead these perturbations interact constructively, as in [4], [6], [24] and [35], whence the limit system is different from the quasi-geostrophic system mentioned above (see $[\mathbf{S}$ ). We aim to study the behavior of strong solutions of $\left[\mathrm{PE}_{\varepsilon}\right]$ in the regime $\varepsilon \rightarrow 0$ in the periodic setting for a large class of tori (see Definition 1.8 which may as well present resonant effects. In particular we prove that the equation $[$ is globally well posed in some suitable space of low-regularity, hence we prove the (global) convergence of solutions of $\left(\mathrm{PE}_{\varepsilon}\right)$ to solutions of $[\mathrm{S}$.

We recall some results on primitive equations. We refer to J.-L. Lions, R. Temam and S. Wang ( [31] and [32]) for the asymptotic expansion of the primitive equations with respect the Rossby number $\varepsilon$ in a spherical and Cartesian geometry.

J.T. Beale and A. J. Bourgeois in 9] study the primitive equations (without viscosity, and with a simplified equation for the density) in a domain which is periodic in the horizontal direction and bounded in the vertical one. By the use of a change of variables they recover a purely periodic setting, on which they prove their result. They study as well the quasi-geostrophic system (fist on short times, then globally) as well as the convergence of primitive equations for regular (i.e. $H^{3}$ ) and well prepared initial data.

In 22 P. Embid and A. Majda present a general formulation for the evolution of geophysical fluids in the periodic setting and derive the limit equation for the kernel part of the solution.

Let us now mention some known result of existence and convergence of solutions for the primitive equations when the spatial domain is $\mathbb{R}^{3}$. In 14 J.-Y. Chemin proved that the solutions of the primitive equations converge toward those of the quasi geostrophic system in the case $F=1$ for regular, well prepared data and under the assumption that $\left|v-v^{\prime}\right|$ (the difference between the diffusion and the thermal diffusivity) is small.

When $F \neq 1$, F. Charve proved in $[10]$ and $[11]$, using dispersive Strichartz estimates, that the solutions of the primitive equations $\left(\mathrm{PE}_{\varepsilon}\right)$ converge globally toward a linear correction of the global solutions of the quasi-geostrophic system QG.

For the inviscid case in the whole space, when $F=1$, we mention the work of D. Iftimie [29] which proves that the potential vorticity $\Omega$ propagates $H^{s}\left(\mathbb{R}^{3}\right), s>5 / 2$ data under the hypothesis $U_{\mathrm{osc}, 0}^{\varepsilon}=o_{\varepsilon}(1)$ in $L^{2}\left(\mathbb{R}^{3}\right)$. If $F \neq 1$, A. Dutrifoy proved in 21$]$ the same result under much weaker assumptions, i.e. $\Omega_{0}$ is a vortex patch and $\left\|U_{0}^{\varepsilon}\right\|_{H^{s}\left(\mathbb{R}^{3}\right)}=\mathcal{O}\left(\varepsilon^{-\gamma}\right), \gamma>$ 0 and small. For the viscid case in the periodic setting I. Gallagher proves in [24] the global convergence of $\left[\mathrm{PE}_{\varepsilon}\right]$ toward QG using a technique introduced by S. Schochet in [39]. Such technique consists in a smart change of variables which cancels some nonlinear interactions which are otherwise impossible to control. We mention at last the work of F. Charve and V.-S. Ngo in 13 for the primitive equation in the whole space for $F \neq 1$ and anisotropic vanishing (horizontal) viscosity.

We recall that the primitive equations and the rotating fluid system

$$
\partial_{t} v+v \cdot \nabla v-v \Delta v+\frac{e^{3} \wedge v}{\varepsilon}=-\nabla p
$$

are intimately connected. In such system the rotation has a stabilizing effect on the solutions of $\left(\overline{R F_{\varepsilon}}\right)$, inducing the fluid to have a strictly columnar dynamic. This was proved at first by E. Grenier in [26] and A. Babin et al. in [4] for the periodic case and by J.-Y. Chemin, B. Desjardins, I. Gallagher and E. Grenier $\mid 16$ in $\mathbb{R}^{3}$. We recall as well the results in 25] in which I. Gallagher and L. Saint-Raymond proved a weak convergence result for weak solutions for fast rotating fluids in which the rotation is inhomogeneous and given by $\frac{1}{\varepsilon} v \wedge b\left(x_{h}\right) e_{3}$.

\subsection{A survey on the notation adopted}

All the vector fields that we consider are real, i.e, we consider applications of the form $V: \mathbb{T}^{3} \rightarrow \mathbb{R}^{4}$. We will often associate to a vector field $V$ the vector field $v: \mathbb{T}^{3} \rightarrow \mathbb{R}^{3}$, which is simply the projection on the first three components of $V$. Moreover all the vector fields considered are periodic in all their components $x_{i}, i=1,2,3$ and have zero global average, i.e. $\int_{\mathbb{T}^{3}} v \mathrm{~d} x=0$, which is equivalent to assume that the first Fourier coefficient $\hat{V}_{0}=0$. We remark that this property is preserved for the Navier-Stokes equations as well as for the primitive equations $\left[\mathrm{PE}_{\varepsilon}\right]$. All the vector fields considered are divergence-free. 
Since the system $\left[\mathrm{PE}_{\varepsilon}\right]$ presents a parabolic behavior in the horizontal directions and an hyperbolic one in the vertical direction we introduce a functional setting which is adapted to describe such anisotropy of the problem. The non-homogeneous Sobolev anisotropic spaces are defined as the closure of $\mathcal{D}\left(\mathbb{T}^{3}\right)$ with respect to the norm

$$
\|u\|_{H^{s, s^{\prime}}\left(\mathbb{T}^{3}\right)}^{2}=\|u\|_{H^{s, s^{\prime}}}^{2}=\sum_{n=\left(n_{h}, n_{3}\right) \in \mathbb{Z}^{3}}\left(1+\left|\check{n_{h}}\right|^{2}\right)^{s}\left(1+\left|\check{n_{3}}\right|^{2}\right)^{s^{\prime}}\left|\hat{u}_{n}\right|^{2},
$$

where we denoted $\check{n}_{i}=n_{i} / a_{i}, \check{n}_{h}=\left(\check{n}_{1}, \check{n}_{2}\right)$ and the Fourier coefficients $\hat{u}_{n}$ are given by $u=\sum_{n} \hat{u}_{n} e^{2 \pi i \check{n} \cdot x}$. In the whole text $\mathcal{F}$ denotes the Fourier transform and $\mathcal{F}^{v}$ the Fourier transform in the vertical variable.

We are interested to study the regularity of the product of two distributions (which is a priori not well defined), in the framework of Sobolev spaces the following product rule can be proved

Lemma 1.1. Let $u, v$ be two distributions with zero average defined on $H^{s}\left(\mathbb{T}^{d}\right)$ and $H^{t}\left(\mathbb{T}^{d}\right)$ respectively, with $s+t>0$, and $s, t<d / 2$, then

$$
\|u \cdot v\|_{H^{s+t-d / 2}\left(\mathbb{T}^{d}\right)} \leqslant C_{s, t}\|u\|_{H^{s}\left(\mathbb{T}^{d}\right)}\|v\|_{H^{t}\left(\mathbb{T}^{d}\right)} \cdot
$$

As in classical isotropic spaces (see [1]) if $s>1 / 2$ the space $H^{s}\left(\mathbb{T}_{v}^{1}\right)$ is a Banach algebra. Combining this fact with the above lemma we deduce the following result which we shall apply all along the paper

Lemma 1.2. Let $u \in H^{s_{1}, s^{\prime}}, v \in H^{s_{2}, s^{\prime}}$ distributions with zero horizontal average with $s_{1}+s_{2}>0, s_{1}, s_{2}<1$ and $s^{\prime}>1 / 2$, then $u \cdot v \in H^{s_{1}+s_{2}-1, s^{\prime}}$ and the following bound holds true

$$
\|u \cdot v\|_{H^{s_{1}+s_{2}-1, s^{\prime}}} \leqslant C\|u\|_{H^{s_{1}, s^{\prime}}}\|v\|_{H^{s_{2}, s^{\prime}}} .
$$

Let us recall as well the definition of the anisotropic Lebesgue spaces. We denote with $L_{h}^{p} L_{v}^{q}$ the space $L^{p}\left(\mathbb{T}_{h}^{2} ; L^{q}\left(\mathbb{T}_{v}^{1}\right)\right)$, defined by the norm:

$$
\|f\|_{L_{h}^{p} L_{v}^{q}}=\|\| f\left(x_{h}, \cdot\right)\left\|_{L^{q}\left(\mathbb{T}_{v}^{1}\right)}\right\|_{L^{p}\left(\mathbb{T}_{h}^{2}\right)}=\left(\int_{\mathbb{T}_{h}^{2}}\left(\int_{\mathbb{T}_{v}^{1}}\left|f\left(x_{h}, x_{3}\right)\right|^{q} \mathrm{~d} x_{3}\right)^{\frac{p}{q}} \mathrm{~d} x_{h}\right)^{\frac{1}{p}} .
$$

In a similar way we define the space $L_{v}^{q} L_{h}^{p}$. It is well-known that the order of integration is important as it is described in the following lemma

Lemma 1.3. Let $1 \leqslant p \leqslant q$ and $f: X_{1} \times X_{2} \rightarrow \mathbb{R}$ a function belonging to $L^{p}\left(X_{1} ; L^{q}\left(X_{2}\right)\right)$ where $\left(X_{1} ; \mu_{1}\right),\left(X_{2} ; \mu_{2}\right)$ are measurable spaces, then $f \in L^{q}\left(X_{2} ; L^{p}\left(X_{1}\right)\right)$ and we have the inequality

$$
\|f\|_{L^{q}\left(X_{2} ; L^{p}\left(X_{1}\right)\right)} \leqslant\|f\|_{L^{p}\left(X_{1} ; L^{q}\left(X_{2}\right)\right)}
$$

In the anisotropic setting the Hölder inequality becomes

$$
\|f g\|_{L_{h}^{p} L_{v}^{q}} \leqslant\|f\|_{L_{h}^{p^{\prime}} L_{v}^{q^{\prime}}}\|g\|_{L_{h}^{p^{\prime \prime}} L_{v}^{q^{\prime \prime}}}
$$

where $1 / p=1 / p^{\prime}+1 / p^{\prime \prime}, 1 / q=1 / q^{\prime}+1 / q^{\prime \prime}$.

\subsection{Results}

We recall at first a result of local existence and uniqueness of solutions for Navier-Stokes equations without vertical viscosity and periodic initial conditions.

Theorem 1.4. Let $s>1 / 2$ and $V_{0} \in H^{0, s}\left(\mathbb{T}^{3}\right)$ a divergence-free vector field. Then there exists a time $T>0$ independent of $\varepsilon$ and a unique solution $V^{\varepsilon}$ for the system $\left[\overline{\mathrm{PE}_{\varepsilon}}\right.$ in the space

$$
V^{\varepsilon} \in \mathcal{C}\left([0, T] ; H^{0, s}\right), \quad \nabla_{h} V^{\varepsilon} \in L^{2}\left([0, T] ; H^{0, s}\right) .
$$

Moreover $\left(V^{\varepsilon}\right)_{\varepsilon>0}$ is uniformly bounded (in $\varepsilon$ ) in the space

$$
V^{\varepsilon} \in L^{\infty}\left(\mathbb{R}_{+} ; L^{2}\left(\mathbb{T}^{3}\right)\right), \quad \nabla_{h} V^{\varepsilon} \in L^{2}\left(\mathbb{R}_{+} ; L^{2}\left(\mathbb{T}^{3}\right)\right) .
$$

The existence part of Theorem 1.4 was proved in [16, while the uniqueness (in the same energy space) was proved in $[30]$.

Remark 1.5. We want to point out that, as it was proved by M. Paicu in 37] (see Proposition 2.7] the maximal lifespan does not depend on the regularity of the initial data, as long as $V_{0} \in H^{0, s}, s>1 / 2$. 
Let $\mathcal{L}(\tau)$ be the semigroup generated by $\mathbb{P} \mathcal{A}$, where $\mathbb{P}$ is the Leray projector on the divergence-free vector fields on the first three components, which leaves unchanged the fourth. In particular the Leray projector in three dimensions is given by the formula $\mathbb{P}^{(3)}=1-\mathcal{R}^{(3)} \otimes \mathcal{R}^{(3)}$, where $\mathcal{R}^{(3)}$ is the three dimensional Riesz transform

$$
\mathcal{R}^{(3)}=\left(\frac{\partial_{1}}{\sqrt{-\Delta}}, \quad \frac{\partial_{2}}{\sqrt{-\Delta}}, \quad \frac{\partial_{3}}{\sqrt{-\Delta}}\right)
$$

while $\mathcal{A}$ is the matrix defined in 1.1 . In the same way we define the operators $\Lambda=\sqrt{-\Delta}, \Lambda_{h}=\sqrt{-\Delta_{h}}, \Lambda_{\nu}=\left|\partial_{3}\right|$. Let $\mathcal{L}(t) V_{0}$ be the unique global solution of

$$
\left\{\begin{array}{l}
\partial_{t} V_{\mathrm{L}}+\mathbb{P} \mathcal{A} V_{\mathrm{L}}=0 \\
\left.V_{\mathrm{L}}\right|_{t=0}=V_{0}
\end{array}\right.
$$

Let us further define $U^{\varepsilon}=\mathcal{L}\left(-\frac{t}{\varepsilon}\right) V^{\varepsilon}$. We will denote $U^{\varepsilon}$ as the sequence of filtered solutions, we define

$$
\mathcal{Q}^{\varepsilon}(U, V)=\mathcal{L}\left(-\frac{t}{\varepsilon}\right) \mathbb{P}\left[\mathcal{L}\left(\frac{t}{\varepsilon}\right) U \cdot \nabla \mathcal{L}\left(\frac{t}{\varepsilon}\right) V\right], \quad \quad \mathbb{D}^{\varepsilon} U=\mathcal{L}\left(-\frac{t}{\varepsilon}\right) \mathbf{D} \mathcal{L}\left(\frac{t}{\varepsilon}\right) U,
$$

where $\mathbf{D}$ is defined in 1.1 , and we consider their limits $\mathcal{Q}, \mathbb{D}$ in $\mathcal{D}^{\prime}$ (we shall see that these limit exists). We can hence formally introduce the limit system

$$
\left\{\begin{array}{l}
\partial_{t} U+\mathcal{Q}(U, U)-\mathbb{D} U=0 \\
\operatorname{div} u=0 \\
\left.U\right|_{t=0}=V_{0}
\end{array}\right.
$$

Since the space domain is periodic resonant effect may play an important role.

Definition 1.6. The resonant set $\mathcal{K}^{\star}$ is the set of frequencies such that

$$
\begin{aligned}
\mathcal{K}^{\star} & =\left\{(k, m, n) \in \mathbb{Z}^{9} \mid \quad \omega^{a}(k)+\omega^{b}(m)=\omega^{c}(n) \text { with } k+m=n, \quad(a, b, c) \in\{-,+\}\right\}, \\
& =\left\{(k, n) \in \mathbb{Z}^{6} \mid \quad \omega^{a}(k)+\omega^{b}(n-k)=\omega^{c}(n), \quad(a, b, c) \in\{-,+\}\right\},
\end{aligned}
$$

where $\omega^{j}, j= \pm$ are the eigenvalues of a suitable operator (see Section 3 for further details). Relatively to the present problem the explicit expression of the eigenvalues is

$$
i \omega^{ \pm}(n)= \pm \frac{i}{F} \frac{\sqrt{\left|\check{n}_{h}\right|^{2}+F^{2} \check{n}_{3}^{2}}}{|\check{n}|}
$$

We may as well associate a resonant space to a determinate frequency $n$, in this case we define

$$
\mathcal{K}_{n}^{\star}=\left\{(k, m) \in \mathbb{Z}^{6} \mid \quad \omega^{a}(k)+\omega^{b}(m)=\omega^{c}(n) \text { with } k+m=n, \quad(a, b, c) \in\{-,+\}\right\} .
$$

Definition 1.7. We say that the torus $\mathbb{T}^{3}$ is non-resonant if $\mathcal{K}^{\star}=\varnothing$.

Tori which are non-resonant, are, generally, a better choice since the oscillating part of the solution satisfies a linear equation (see [24]). Indeed though a generic torus may as well present resonant effects. For this reason we introduce the following definition:

Definition 1.8. We say that a torus $\mathbb{T}^{3} \subset \mathbb{R}^{3}$ satisfies the condition $(\mathcal{P})$ if either one or the other of the following conditions is satisfied:

1. $\mathbb{T}^{3}$ is non-resonant.

2. If $\mathbb{T}^{3}$ is resonant, the Froude number $F^{2}$ is rational, and either

- $a_{3}^{2} / a_{1}^{2} \in \mathbb{Q}$ and $a_{3}^{2} / a_{2}^{2}$ is not algebraic of degree smaller or equal than four.

- $a_{3}^{2} / a_{2}^{2} \in \mathbb{Q}$ and $a_{3}^{1} / a_{2}^{2}$ is not algebraic of degree smaller or equal than four.

Remark 1.9. The above definition (Definition 1.8) is motivated in Section 5.1 The point 2 ensures that even with resonant effects we can propagate the horizontal average of the initial data, thing that, generally, is not true for threedimensional Navier-Stokes equations.

Although $[S$ is an hyperbolic system in the vertical variable we are able to prove that there exist weak (in the sense of distributions) global solutions. This was first remarked by M. Paicu in [35] and it due to the fact that the limit bilinear form $\mathcal{Q}$ has in fact better product rules than the standard bilinear transport form (see as well Lemma 8.4). The complete statement of the theorem is the following one. 
Theorem 1.10. Let $\mathbb{T}^{3}$ be a 3-dimensional torus in $\mathbb{R}^{3}$ and let $F \neq 1$, for each divergence-free vector field $V_{0} \in L^{2}\left(\mathbb{T}^{3}\right)$ and $\Omega_{0}=-\partial_{2} v_{0}^{1}+\partial_{1} v_{0}^{2}-F \partial_{3} T_{0} \in L^{2}\left(\mathbb{T}^{3}\right)$ there exists a distributional solution of the system

$$
\left\{\begin{array}{l}
\partial_{t} U+\mathcal{Q}(U, U)-\mathbb{D} U=0 \\
\operatorname{div} u=0 \\
\left.U\right|_{t=0}=V_{0}
\end{array}\right.
$$

in the space $\mathcal{D}^{\prime}\left(\mathbb{R}_{+} \times \mathbb{T}^{3}\right)$ which moreover belongs to the space

$$
U \in L^{\infty}\left(\mathbb{R}_{+} ; L^{2}\left(\mathbb{T}^{3}\right)\right), \quad \nabla_{h} U \in L^{2}\left(\mathbb{R}_{+} ; L^{2}\left(\mathbb{T}^{3}\right)\right)
$$

and satisfies the following energy estimate

$$
\|U(t)\|_{L^{2}\left(\mathbb{T}^{3}\right)}^{2}+2 c \int_{0}^{t}\left\|\nabla_{h} U(s)\right\|_{L^{2}\left(\mathbb{T}^{3}\right)}^{2} \mathrm{~d} s \leqslant\left\|U_{0}\right\|_{L^{2}\left(\mathbb{T}^{3}\right)}^{2},
$$

where the constant $c=\min \left\{v_{h}, v_{h}^{\prime}\right\}>0$.

We remark that Theorem 1.10 holds for any three-dimensional torus. We do not require the condition $[(\mathcal{P})$ to hold.

A natural question we address to is whether the system $\left[\overline{\mathrm{PE}_{\varepsilon}}\right.$ converges (even in a weak sense) to the limit system $(\mathrm{S})$ as $\varepsilon \rightarrow 0$. This is the scope of the following theorem:

Theorem 1.11. Let the initial data $V_{0}$ be as in Theorem 1.10 then defining the operator

$$
\mathcal{L}(\tau)=e^{-\tau \mathbb{P} \mathcal{A}}
$$

and denoting as $U$ the distributional solution of the limit system $[\mathrm{S}]$ identified in Theorem 1.10 the following convergence holds in the sense of distributions

$$
V^{\varepsilon}-\mathcal{L}\left(\frac{t}{\varepsilon}\right) U \stackrel{\varepsilon \rightarrow 0}{\longrightarrow} 0
$$

Moreover $U$ weak solution of the limit system $[\mathrm{S}]$ can be described as the superposition of the evolution of $U=U_{\mathrm{QG}}+$ $U_{\mathrm{osc}}=V_{\mathrm{QG}}+U_{\mathrm{osc}}$ where $V_{\mathrm{QG}}$ solves

$$
\left\{\begin{array}{l}
\partial_{t} V_{\mathrm{QG}}+a_{\mathrm{QG}}\left(D_{h}\right) V_{\mathrm{QG}}=-\left(\begin{array}{c}
\nabla_{h}^{\perp} \\
0 \\
-F \partial_{3}
\end{array}\right) \Delta_{F}^{-1}\left(v_{\mathrm{QG}}^{h} \cdot \nabla_{h} \Omega\right) \\
\operatorname{div}_{h} v_{\mathrm{QG}}^{h}=\operatorname{div} v_{\mathrm{QG}}=0 \\
\left.V_{\mathrm{QG}}\right|_{t=0}=V_{\mathrm{QG}, 0}
\end{array}\right.
$$

and $U_{\mathrm{osc}}$ solves

$$
\left\{\begin{array}{l}
\partial_{t} U_{\mathrm{osc}}+\mathcal{Q}\left(V_{\mathrm{QG}}, U_{\mathrm{osc}}\right)+\mathcal{Q}\left(U_{\mathrm{osc}}, V_{\mathrm{QG}}\right)+\mathcal{Q}\left(U_{\mathrm{osc}}, U_{\mathrm{osc}}\right)+a_{\mathrm{osc}}\left(D_{h}\right) U_{\mathrm{osc}}=0 \\
\operatorname{div} u_{\mathrm{osc}}=0 \\
\left.U_{\mathrm{osc}}\right|_{t=0}=U_{\mathrm{osc}, 0}=\left(V_{0}\right)_{\mathrm{osc}}
\end{array}\right.
$$

The operators $a_{\mathrm{QG}}$ and $a_{\mathrm{Osc}}$ are elliptic in the horizontal variables, in the sense that there exists a positive constant $c>o$ such that

$$
\left(a_{\mathrm{QG}}\left(D_{h}\right) u \mid u\right)_{L^{2}},\left(a_{\mathrm{osc}}\left(D_{h}\right) u \mid u\right)_{L^{2}} \geqslant c\left\|\nabla_{h} u\right\|_{L^{2}}^{2},
$$

and $\mathcal{Q}$ is a bilinear form which shares many aspects with the more classical transport form, but has better properties as far as the regularity of the product is concerned (see Section 8.2 for the product rules and 3.14 for the proper definition of $\mathcal{Q}$ ).

Performing some a priori estimates on the limit system [S] we can improve of the above theorem, at the cost of having well prepared initial data and tori which satisfy Condition $(\mathcal{P})$. We say that a data $V_{0}$ is well prepared if it has zero horizontal mean, i.e. $\int_{\mathbb{T}_{h}^{2}} V_{0}\left(x_{h}, x_{3}\right) \mathrm{d} x_{h}=0$. This property is conserved by the limit system (S) as long as the condition $(\mathcal{P})$ is satisfied (see Lemma 5.6 5.7. Moreover we ask as well that the potential vorticity, defined as

$$
\Omega(t, x)=-\partial_{2} U^{1}(t, x)+\partial_{1} U^{2}(t, x)-F \partial_{3} U^{4}(t, x),
$$

belongs to $H^{0, s}, s \geqslant 0$ at time $t=0$. 
Theorem 1.12. Let $\mathbb{T}^{3}$ satisfy the condition $\left[(\mathcal{P})\right.$ and consider a divergence-free vector field $U_{0} \in H^{0, s}$ with zero horizontal average. Let $\Omega_{0} \in H^{0, s}$, for $s \geqslant 1$ and $F \neq 1$, the global weak solution of $[S$ is in fact strong and it belongs to the space of sub-critical regularity

$$
U \in L^{\infty}\left(\mathbb{R}_{+} ; H^{0, s}\right), \quad \nabla_{h} U \in L^{2}\left(\mathbb{R}_{+} ; H^{0, s}\right) .
$$

Moreover it satisfies, for each $t>0$, the energy bound

$$
\|U(t)\|_{H^{0, s}}^{2}+c \int_{0}^{t}\left\|\nabla_{h} U(s)\right\|_{H^{0, s}}^{2} \leqslant \mathcal{E}\left(\left\|U_{0}\right\|_{H^{0, s}}^{2}\right)
$$

where $\mathcal{E}$ is a suitable function which is bounded on compact sets. The solution $U$ is unique in the space $L^{\infty}\left(\mathbb{R}_{+} ; H^{0, \sigma}\right) \cap$ $L^{2}\left(\mathbb{R}_{+}, H^{1, \sigma}\right)$ for $\sigma \in[-1 / 2, s)$.

Remark 1.13. Compared to the work of M. Paicu [35] the author requires only $s>1 / 2$. This discrepancy is due to the fact that in the present work the limit system is well-posed only for $s \geqslant 1$. Indeed we are able to propagate $H^{0, s}, s \geqslant 0$ norms for the potential vorticity $\Omega$, and, as explained in Lemma 5.5 . $\left\|V_{\mathrm{QG}}\right\|_{H^{0, s+1}} \lesssim\|\Omega\|_{H^{0, s}}$.

The main idea in the propagation of regularity stated in Theorem 1.12 is that we can recover the missing viscosity in the vertical direction using the fact that the vector field $u$ is divergence-free. We can in fact observe that in the nonlinear term the vertical derivative is always multiplied by the third component $u^{3}$ of the vector field considered (i.e. terms of the form $u^{3} \partial_{3}$ ). We hence remark the fact that the term $\partial_{3} u^{3}$ is more regular thanks to the relation $-\partial_{3} u^{3}=\operatorname{div}_{h} u^{h}$, and due to the fact that the horizontal viscosity has a regularizing effect on the derivatives in the horizontal variable $x_{h}$.

Theorem 1.14. Let $\mathbb{T}^{3}$ satisfy the condition $(\mathcal{P}), \Omega_{0}=-\partial_{2} v_{0}^{1}+\partial_{1} v_{0}^{2}-F \partial_{3} T_{0} \in H^{0, s}$ and $V_{0} \in H^{0, s}$ for $s>1$ a divergence free vector field. Let $V^{\varepsilon}$ be a local solution of $\left[\mathrm{PE}_{\varepsilon}\right]$ and $U$ be the unique global solution of the limiti system $[\mathrm{S}]$. Then the following convergences take place

$$
\begin{aligned}
\lim _{\varepsilon \rightarrow 0}\left(V^{\varepsilon}-\mathcal{L}\left(\frac{t}{\varepsilon}\right) U\right) & =0 & & \text { in } \mathcal{C}\left(\mathbb{R}_{+} ; H^{0, \sigma}\right), \\
\lim _{\varepsilon \rightarrow 0} \nabla_{h}\left(V^{\varepsilon}-\mathcal{L}\left(\frac{t}{\varepsilon}\right) U\right) & =0 & & \text { in } L^{2}\left(\mathbb{R}_{+} ; H^{0, \sigma}\right),
\end{aligned}
$$

for $\sigma \in[1, s)$.

The paper is divided as follows

- In Section 2 we introduce some mathematical tools that will be useful in the development of the paper.

- Section 3 we provide a careful analysis of the spectral properties of the linear system whose evolution is determined by the operator $\mathbb{P} \mathcal{A}$. In Subsection 3.1]we state some results proved in [22], [23] and [5] which describe the behavior of the limit bilinear interaction $\mathcal{Q}(U, U)$ in $(S)$ along the eigendirections spanned by the eigenvectors of $\mathbb{P} \mathcal{A}$.

- In Section 4 we prove Theorem 1.10. Such result is not a straightforward application of Leray Theorem since, due to the lack of the vertical diffusivity, the solutions are bounded in the space $L_{\text {loc }}^{2}\left(\mathbb{R}_{+} ; H^{1,0}\right)$ only. Such space is not compactly embedded in $L_{\text {loc }}^{2}\left(\mathbb{R}_{+} ; L^{2}\right)$, this prevents us to use standard compactness theorems in functional spaces such as Aubin-Lions lemma (see [3]). Nonetheless using Fujiwara near-optimal bound (see [33]) we can transform a vertical derivative $\partial_{3}$ in a multi-index of the form $C\left(\partial_{1}^{N_{1}}, \partial_{2}^{N_{2}}\right)$, where $N_{1}, N_{2}$ may as well be large. The system $\overline{\mathrm{PE}_{\varepsilon}}$ has a non-zero diffusive effects in the horizontal directions, hence we can prove that bilinear interactions of weakly converging (in the sense that converge w.r.t. a Sobolev topology of negative index) sequences converge in the sense of distributions to some limit element.

- In Section 5 we prove Theorem 1.11 The approach is twofold:

- Thanks to a topological argument we prove that the sequence $\left(V^{\varepsilon}\right)_{\varepsilon>0}$ is compact in some weak sense,

- A careful analysis of the bilinear interactions in the limit $\varepsilon \rightarrow 0$ gives us the explicit form of the bilinear limit interactions.

Next in Subsection 5.1 we prove that, under some suitable geometric conditions (see Definition 1.8) the limit system [S] propagates globally-in-time the horizontal average of the initial data.

- In Section 6 we prove that the limit system propagates globally-in-time $H^{0, s}$ data, at the price of having well prepared (in the sense of zero-horizontal average) initial data and domains which satisfy the condition $(\mathcal{P})$ given in Definition 1.8 . Hence we prove Theorem 1.12 .

- Lastly in Section 7 we prove Theorem 1.14 i.e. that we can approximate globally the solutions of $\left[\mathrm{PE}_{\varepsilon}\right]$ as $\varepsilon \rightarrow 0$ with the (global) solutions of [S] in some suitable subcritical topology. 


\section{Preliminaries}

This section is devoted to introduce the mathematical tools that will be used all along the paper and which are necessary to understand the contents described in the following pages.

\subsection{Elements of Littlewood-Paley theory}

Let us define the (non-homogeneous) vertical dyadic blocks as follows:

$$
\begin{aligned}
\triangle_{q}^{\mathrm{v}} u & =\sum_{n \in \mathbb{Z}^{3}} \hat{u}_{n} \varphi\left(\frac{\left|\check{n}_{3}\right|}{2^{q}}\right) e^{i \check{n} \cdot x} & & \text { for } q \geqslant 0, \\
\triangle_{-1}^{v} u & =\sum_{n \in \mathbb{Z}^{3}} \hat{u}_{n} \chi\left(\left|\check{n}_{3}\right|\right) e^{i \check{n} \cdot x}, & & \\
\triangle_{q}^{\mathrm{v}} u & =0 & & \text { for } q \leqslant-2,
\end{aligned}
$$

where $u \in \mathcal{D}^{\prime}\left(\mathbb{T}^{3}\right)$ and $\hat{u}_{n}$ are the Fourier coefficients of $u$. The functions $\varphi$ and $\chi$ are smooth functions with compact support such that

$$
\operatorname{Supp} \chi \subset B\left(0, \frac{4}{3}\right), \quad \operatorname{Supp} \varphi \subset \mathcal{C}\left(\frac{3}{4}, \frac{8}{3}\right)
$$

And for each $t \in \mathbb{R}$ the sequence $\left(\chi(\cdot), \varphi\left(2^{-q} \cdot\right)\right)_{q \in \mathbb{N}}$ is a partition of the unity. Let us define further the vertical cut-off operator as $S_{q}^{v} u=\sum_{q^{\prime} \leqslant q-1} \triangle_{q^{\prime}}^{\mathrm{v}} u$.

\subsection{Anisotropic paradifferential calculus}

We can, at least formally, write for two distributions $u$ and $v$

$$
u=\sum_{q \in \mathbb{Z}} \triangle_{q}^{\mathrm{v}} u ; \quad v=\sum_{q^{\prime} \in \mathbb{Z}} \triangle_{q^{\prime}}^{\mathrm{v}} v ; \quad u v=\sum_{\substack{q \in \mathbb{Z} \\ q^{\prime} \in \mathbb{Z}}} \triangle_{q}^{\mathrm{v}} u \triangle_{q^{\prime}}^{\mathrm{v}} v
$$

We are going to perform a Bony decomposition in the vertical variable (see [7], [8, [15] for the isotropic case and [16], [28] for the anisotropic one).

Paradifferential calculus is a mathematical tool for splitting the above sum in three parts

$$
u v=T_{u}^{v} v+T_{v}^{v} u+R^{v}(u, v)
$$

where

$$
T_{u}^{v} v=\sum_{q} S_{q-1}^{v} u \triangle_{q}^{\mathrm{v}} v, \quad T_{\nu}^{v} u=\sum_{q^{\prime}} S_{q^{\prime}-1}^{v} v \triangle_{q^{\prime}}^{\mathrm{v}} u, \quad R^{v}(u, v)=\sum_{k} \sum_{|\mu| \leqslant 1} \triangle_{k}^{v} u \triangle_{k+\mu}^{v} \nu .
$$

In particular the following almost orthogonality properties hold

$$
\begin{array}{ll}
\triangle_{q}^{\mathrm{v}}\left(S_{q^{\prime}-1}^{\mathrm{v}} a \triangle_{q^{\prime}}^{\mathrm{v}} b\right)=0 & \text { if }\left|q-q^{\prime}\right| \geqslant 5, \\
\triangle_{q}^{\mathrm{v}}\left(\triangle_{q^{\prime}}^{\mathrm{v}} a \triangle_{q^{\prime}+\mu}^{v} b\right)=0 & \text { if } q^{\prime}<q-4,|\mu| \leqslant 1,
\end{array}
$$

and hence we will often use the following relation

$$
\begin{aligned}
& \triangle_{q}^{\mathrm{v}}(u \nu)=\sum_{\left|q-q^{\prime}\right| \leqslant 4} \Delta_{q}^{\mathrm{v}}\left(S_{q^{\prime}-1}^{\nu} \nu \triangle_{q^{\prime}}^{\mathrm{v}} u\right)+\sum_{\left|q-q^{\prime}\right| \leqslant 4} \triangle_{q}^{\mathrm{v}}\left(S_{q^{\prime}-1}^{v} u \triangle_{q^{\prime}}^{\mathrm{v}} \nu\right)+\sum_{q^{\prime} \geqslant q-4|\mu| \leqslant 1} \sum_{q} \triangle_{q}^{\mathrm{v}}\left(\triangle_{q^{\prime}}^{\mathrm{v}} u \triangle_{q^{\prime}+\mu}^{v} \nu\right), \\
& =\sum_{\left|q-q^{\prime}\right| \leqslant 4} \triangle_{q}^{\mathrm{v}}\left(S_{q^{\prime}-1}^{v} v \triangle_{q^{\prime}}^{\mathrm{v}} u\right)+\sum_{q^{\prime}>q-4} \triangle_{q}^{\mathrm{v}}\left(S_{q^{\prime}+2}^{v} u \triangle_{q^{\prime}}^{\mathrm{v}} v\right) \text {. }
\end{aligned}
$$

In the paper [19] J.-Y. Chemin and N. Lerner introduced the following asymmetric decomposition, which was first used by J.-Y. Chemin et al. in [16] in its anisotropic version. This particular decomposition turns out to be very useful in our context

$$
\triangle_{q}^{\mathrm{v}}(u v)=S_{q-1}^{v} u \triangle_{q}^{\mathrm{v}} v+\sum_{\left|q-q^{\prime}\right| \leqslant 4}\left\{\left[\triangle_{q}^{\mathrm{v}}, S_{q^{\prime}-1}^{\mathrm{v}} u\right] \triangle_{q^{\prime}}^{\mathrm{v}} v+\left(S_{q}^{v} u-S_{q^{\prime}-1}^{\mathrm{v}} u\right) \triangle_{q}^{\mathrm{v}} \Delta_{q^{\prime}}^{\mathrm{v}} v\right\}+\sum_{q^{\prime}>q-4} \triangle_{q}^{\mathrm{v}}\left(S_{q^{\prime}+2}^{v} v \triangle_{q^{\prime}}^{\mathrm{v}} u\right),
$$

where the commutator $\left[\triangle_{q}^{\mathrm{v}}, a\right] b$ is defined as $\left[\triangle_{q}^{\mathrm{v}}, a\right] b=\triangle_{q}^{\mathrm{v}}(a b)-a \triangle_{q}^{\mathrm{v}} b$. 
We denote as $\left(b_{q}\right)_{q \geqslant-1}$ any sequence which is summable that may depend on different parameters such that $\sum_{q} b_{q} \leqslant 1$. In the same way we denote as $\left(c_{q}\right)_{q} \in \ell^{2}(\mathbb{Z})$ any sequence such that $\sum_{q} c_{q}^{2} \leqslant 1$. As well $C$ is a (large) positive constant independent of any parameter and $c$ a small one, these two constants may differ implicitly from line to line. We remark that the regularity of a function can be rephrased in the following way: we say that $u \in H^{0, s}$ only if there exists a sequence $\left(c_{q}\right)_{q}$ depending on $u$ such that

$$
\left\|\triangle_{q}^{v} u\right\|_{L^{2}\left(\mathbb{J}^{3}\right)} \leqslant C c_{q}(u) 2^{-q s}\|u\|_{H^{0, s}}
$$

\subsection{Dyadic blocks and commutators as convolution operators}

The dyadic blocks and the low-frequencies truncation operators can be seen as convolution operators, in particular if we denote as $h=\mathcal{F}^{-1} \varphi$ and $g=\mathcal{F}^{-1} \chi$ we have

$$
\begin{aligned}
& \triangle_{q}^{\mathrm{v}} u=\varphi\left(2^{-q} D\right) u=2^{q} \int_{\mathbb{T}} h\left(2^{q} y\right) u(x-y) \mathrm{d} y, \\
& S_{q}^{v} u=\chi\left(2^{-q} D\right) u=2^{q} \int_{\mathbb{T}} g\left(2^{q} y\right) u(x-y) \mathrm{d} y .
\end{aligned}
$$

This is due to the fact that $\triangle_{q}^{\mathrm{v}} u(x)=\left(\mathcal{F}^{\nu}\right)^{-1}(\varphi(\cdot) \hat{u}(\cdot))(x)$. In particular we want to express a commutator as a convolution operator, since a commutator is defined as

$$
\left[\triangle_{q}^{\mathrm{v}}, a\right] b(x)=\triangle_{q}^{\mathrm{v}}(a b)(x)-a(x) \triangle_{q}^{\mathrm{v}} b(x),
$$

and we apply to the right hand side of the above equation the relation in 2.5], we obtain that

$$
\left[\triangle_{q}^{\mathrm{v}}, a\right] b(x)=2^{q} \int_{\mathbb{T}} h\left(x_{h}, x_{3}-y_{3}\right)\left(a\left(x_{h}, y_{3}\right)-a\left(x_{h}, x_{3}\right)\right) b\left(x_{h}, y_{3}\right) \mathrm{d} y_{3} .
$$

Thanks to Taylor expansion with reminder in Cauchy form we know that

$$
a\left(x_{h}, y_{3}\right)-a\left(x_{h}, x_{3}\right)=\partial_{3} a\left(x_{h}, x_{3}+\tau\left(x_{3}-y_{3}\right)\right)\left(x_{3}-y_{3}\right),
$$

for some $\tau \in(0,1)$, hence we can write the commutator as

$$
\left[\triangle_{q}^{\mathrm{v}}, a\right] b(x)=2^{q} \int_{\mathbb{T}}\left(x_{3}-y_{3}\right) h\left(x_{h}, x_{3}-y_{3}\right) \partial_{3} a\left(x_{h}, x_{3}+\tau\left(x_{3}-y_{3}\right)\right) b\left(x_{h}, y_{3}\right) \mathrm{d} y_{3} .
$$

\subsection{Some basic estimates}

The interest in the use of the dyadic decomposition is that the derivative in the vertical direction of a function localized in vertical frequencies of size $2^{q}$ acts like the multiplication of a factor $2^{q}$ (up to a constant independent of $q$ ). In our setting (periodic case) a Bernstein type inequality holds. For a proof of the following lemma we refer to the work 28.

Lemma 2.1. Let $u$ be a function such that $\operatorname{supp} \mathcal{F}^{v} u \subset \mathbb{T}_{h}^{2} \times 2^{q} \mathcal{C}$, where $\mathcal{F}^{v}$ denotes the Fourier transform in the vertical variable. For all integers $k, p \in[1, \infty], 1 \leqslant r^{\prime} \leqslant r \leqslant \infty$, the following relations hold

$$
\begin{aligned}
& 2^{q k} C^{-k}\|u\|_{L_{h}^{p} L_{v}^{r}} \leqslant\left\|\partial_{x_{3}}^{k} u\right\|_{L_{h}^{p} L_{v}^{r}} \leqslant 2^{q k} C^{k}\|u\|_{L_{h}^{p} L_{v}^{r}}, \\
& 2^{q k} C^{-k}\|u\|_{L_{v}^{r} L_{h}^{p}} \leqslant\left\|\partial_{x_{3}}^{k} u\right\|_{L_{v}^{r} L_{h}^{p}} \leqslant 2^{q k} C^{k}\|u\|_{L_{v}^{r} L_{h}^{p} .}
\end{aligned}
$$

Let now $\infty \geqslant r \geqslant r^{\prime} \geqslant 1$ be real numbers. Let supp $\mathcal{F}^{v} u \subset \mathbb{T}_{h}^{2} \times 2^{q} B$, then

$$
\begin{aligned}
& \|u\|_{L_{h}^{p} L_{v}^{r}} \leqslant C 2^{q\left(\frac{1}{r^{\prime}}-\frac{1}{r}\right)}\|u\|_{L_{h}^{p} L_{v}^{r^{\prime}}}, \\
& \|u\|_{L_{v}^{r} L_{h}^{p}} \leqslant C 2^{q\left(\frac{1}{r^{\prime}}-\frac{1}{r}\right)}\|u\|_{L_{v}^{r^{\prime}} L_{h}^{p}} .
\end{aligned}
$$

The following are inequalities of Gagliardo-Niremberg type, we will avoid to give the proofs of such tools since they are already present in [35].

Lemma 2.2. There exists a constant $C$ such that for all periodic vector fields $u$ on $\mathbb{T}^{3}$ with zero horizontal average $\left(\int_{\mathbb{T}_{h}^{2}} u\left(x_{h}, x_{3}\right) \mathrm{d} x_{h}=0\right)$ we have

$$
\|u\|_{L_{v}^{2} L_{h}^{4}} \leqslant C_{1}\|u\|_{H^{1 / 2,0}} \leqslant C_{2}\|u\|_{L^{2}\left(\mathbb{T}^{3}\right)}^{1 / 2}\left\|\nabla_{h} u\right\|_{L^{2}\left(\mathbb{T}^{3}\right)}^{1 / 2} .
$$


From Lemma 2.1 and 2.7] we can deduce the following result

Corollary 2.3. Let $u$ be a periodic vector field such that Supp $\mathcal{F}^{v} u \subset \mathbb{T}_{h}^{2} \times 2^{q} B$, then

$$
\|u\|_{L_{v}^{\infty} L_{h}^{2}} \leqslant C 2^{q / 2}\|u\|_{L^{2}\left(\mathbb{T}^{3}\right)},
$$

moreover if $u$ has zero horizontal average

$$
\|u\|_{L_{\nu}^{\infty} L_{h}^{4}} \leqslant C 2^{q / 2}\|u\|_{L^{2}\left(\mathbb{T}^{3}\right)}^{1 / 2}\left\|\nabla_{h} u\right\|_{L^{2}\left(\mathbb{T}^{3}\right)}^{1 / 2},
$$

Lemma 2.4. Let $s$ be a real number and $\mathbb{t}^{3}$ a three dimensional torus. For all vector fields $u$ with zero horizontal average, the following inequality holds

$$
\|u\|_{H^{1 / 2, s}} \leqslant C\|u\|_{H^{0, s}}^{1 / 2}\left\|\nabla_{h} u\right\|_{H^{0, s}}^{1 / 2} .
$$

Corollary 2.5. Let $s>1 / 2$. There exists a constant $C$ such that the inequality

$$
\|u\|_{L_{v}^{\infty} L_{h}^{2}} \leqslant C\|u\|_{H^{0, s}}
$$

holds. Moreover if u is of zero horizontal average we have

$$
\|u\|_{L_{v}^{\infty} L_{h}^{4}} \leqslant C\|u\|_{H^{0, s}}^{1 / 2}\left\|\nabla_{h} u\right\|_{H^{0, s}}^{1 / 2} .
$$

Finally we state a lemma that shows that the commutator with the dyadic block in the vertical frequencies is a regularizing operator. The proof of such lemma can be found in [37].

Lemma 2.6. Let $\mathbb{T}^{3}$ be a three-dimensional torus and $p, r, s$ real positive numbers such that $\infty \geqslant r^{\prime}, s^{\prime}, p, r, s \geqslant 1 \frac{1}{r^{\prime}}+\frac{1}{s^{\prime}}=$ $\frac{1}{2}$ and $\frac{1}{p}=\frac{1}{r}+\frac{1}{s}$. There exists a constant $C$ such that for all vector fields $u$ and $v$ on $\mathbb{T}^{3}$ we have the inequality

$$
\left\|\left[\triangle_{q}^{\mathrm{v}}, u\right] v\right\|_{L_{v}^{2} L_{h}^{p}} \leqslant C 2^{-q}\left\|\partial_{3} u\right\|_{L_{v}^{r^{\prime}} L_{h}^{r}}\|v\|_{L_{\nu}^{s^{\prime}} L_{h}^{s}}
$$

\subsection{Preliminary results on the Navier-Stokes equations with zero vertical diffusivity}

A primary tool in the study of the convergence of the primitive equations $[\overline{\mathrm{PE}} \bar{\varepsilon}]$ to the limit system $[\mathrm{S}]$ will be a careful study of the Navier-Stokes equation with only horizontal diffusion

$$
\left\{\begin{array}{l}
\partial_{t} v+v \cdot \nabla v-v_{h} \Delta_{h} v+\nabla p=0 \quad \text { in } \quad \mathbb{R}_{+} \times \mathbb{T}^{3} \\
\operatorname{div} v=0 \\
\left.v\right|_{t=0}=v_{0}
\end{array}\right.
$$

This equation in the case of the periodic data on $\mathbb{T}^{3}$ has been carefully studied in 37 , hence we will refer to this work as we go along.

Given any vector field $A$ we denote

$$
\underline{A}\left(x_{3}\right)=\frac{1}{\left|\mathbb{T}_{h}^{2}\right|} \int_{\mathbb{T}_{h}^{2}} A\left(y_{h}, x_{3}\right) \mathrm{d} y_{h},
$$

and

$$
\tilde{A}\left(x_{h}, x_{3}\right)=A\left(x_{h}, x_{3}\right)-\underline{A}\left(x_{3}\right) .
$$

Here we start giving the following energy estimate for three-dimensional anisotropic Navier-Stokes equations

Proposition 2.7. Let $s \geqslant s_{0}>\frac{1}{2}$ and $v$ a solution of $\left[\mathrm{NS}_{h}\right]$ belonging to the space $\mathcal{C}\left([0, T] ; H^{0, s}\right)$ whose horizontal gradient $\nabla_{h} v \in L^{2}\left([0, T] ; H^{0, s}\right)$. Let us suppose moreover that $v=\underline{v}+\tilde{v}$ where $\underline{v}$ is the horizontal average of $v$ and $\tilde{v}$ has $z e r o$ horizontal mean. Suppose moreover that $\|\underline{v}(t)\|_{H_{v}^{s_{0}}} \leqslant c a_{3}^{-1} \overline{v_{h}}$ in $[0, T]$, then for $t \in[0, T]$

$$
\|v(t)\|_{H^{0, s}}^{2}+v_{h} \int_{0}^{t}\left\|\nabla_{h} v(\tau)\right\|_{H^{0, s}}^{2} \mathrm{~d} \tau \leqslant\left\|v_{0}\right\|_{H^{0, s}}^{2} \exp \left(C \int_{0}^{t}\left\|\nabla_{h} v(\tau)\right\|_{H^{0, s_{0}}}^{2} \mathrm{~d} \tau+C \int_{0}^{t}\|v(\tau)\|_{H^{0, s_{0}}}^{2}\left\|\nabla_{h} \nu(\tau)\right\|_{H^{0, s_{0}}}^{2}\right) .
$$

For a proof of Proposition 2.7 we refer to the works [35. Proposition 3.1] and [37].

Remark 2.8. Proposition 2.7 was proved by M. Paicu for $s \geqslant s_{0}>\frac{1}{2}$ in 35 . Indeed in $35 \mid$ the limit system was a coupling between a $2 \mathrm{~d}$ Navier-Stokes system and the oscillating part. Indeed the $2 \mathrm{~d}$ Navier-Stokes system is globally well posed if the initial data depends on $x_{h}$ only and it is in $H^{0, s}$ for $s \geqslant 0$. The oscillating part instead is globally well posed in $H^{0, s}$ for $s>1 / 2$. In our case though the limit flow is the sum of $V_{\mathrm{QG}}$ satisfying [5.11] and the oscillating part $U_{\text {osc }}$ which are two three-dimensional vector fields. Now, $U_{\text {osc }}$ is globally well posed in $H^{0, s}$ for $s>1 / 2$ (see Proposition 6.5), but $V_{\mathrm{QG}}$ is globally well posed in $H^{0, s}$ for $s \geqslant 1$ (see Proposition 6.2 and Lemma 5.5. This is why in the following as long as we are required to apply Proposition 2.7 we shall use the index $s_{0}>1$ instead that $s_{0}>1 / 2$. 
Proposition 2.9. Let $s>\frac{1}{2}$ and $\mathbb{T}^{3}$ an arbitrary torus and $w \in \mathcal{C}\left([0, T] ; H^{0, s}\right), \nabla_{h} w \in L^{2}\left([0, T] ; H^{0, s}\right)$ a solution of the problem

$$
\left\{\begin{array}{l}
\partial_{t} w+w \cdot \nabla w+u \cdot \nabla w+w \cdot \nabla u-v_{h} \Delta_{h} w+\nabla p=f \\
\operatorname{div} w=0 \\
\left.w\right|_{t=0}=w_{0}
\end{array}\right.
$$

where $u \in \mathcal{C}\left([0, T] ; H^{0, s}\right), \nabla_{h} u \in L^{2}\left([0, T] ; H^{0, s}\right)$ a divergence-free vector field such that its horizontal average satisfies $\|\underline{u}(t)\|_{H_{v}^{s}} \leqslant c a_{3}^{-1} v_{h}$ for all $t \in[0, T]$ and $f=\underline{f}+\tilde{f}$ is such that

$$
\begin{aligned}
& \underline{f} \in L^{1}\left([0, T] ; H_{\nu}^{-\frac{1}{2}}\right), \\
& \tilde{f} \in L^{2}\left([0, T] ; H^{-1,-\frac{1}{2}}\right) .
\end{aligned}
$$

Then there exists a constant $C>0$ such that, for all $t \in[0, T]$,

$$
\begin{aligned}
\|w(t)\|_{H^{0,-\frac{1}{2}}}^{2} & +v_{h} \int_{0}^{t}\left\|\nabla_{h} w(s)\right\|_{H^{0,-\frac{1}{2}}}^{2} \mathrm{~d} s \\
& \leqslant C\left(\left\|w_{0}\right\|_{H^{0,-\frac{1}{2}}}^{2}+\int_{0}^{t}\|\tilde{f}(s)\|_{H^{-1,-\frac{1}{2}}}^{2} \mathrm{~d} s+\int_{0}^{t}\|\underline{f}(s)\|_{H_{v}^{-\frac{1}{2}}} \mathrm{~d} s\right) \\
& \times \exp \left\{\int_{0}^{t}\|\underline{f}(s)\|_{H_{v}^{-\frac{1}{2}}} \mathrm{~d} s+\int_{0}^{t}\left(1+\|w(s)\|_{H^{0, s}}^{2}\right)\left\|\nabla_{h} w(s)\right\|_{H^{0, s}}^{2} \mathrm{~d} s+\int_{0}^{t}\left(1+\|u(s)\|_{H^{0, s}}^{2}\right)\left\|\nabla_{h} u(s)\right\|_{H^{0, s}}^{2} \mathrm{~d} s\right\} .
\end{aligned}
$$

Proof. [35. Proposition 3.2, p. 182]

Remark 2.10. Let us remark the fact that we impose two different kind of regularities on the exterior force. In order to obtain global results in time we shall apply this proposition for bulk forces which are $f \in L^{1}\left(\mathbb{R}_{+}, H^{-1,-1 / 2}\right) \cap$ $L^{2}\left(\mathbb{R}_{+}, H^{-1,-1 / 2}\right)$.

\section{The filtering operator $\mathbb{P} \mathcal{A}$}

Let us consider the following linear equation

$$
\left\{\begin{array}{l}
\partial_{t} V_{\mathrm{L}}+\mathbb{P} \mathcal{A} V_{\mathrm{L}}=0 \\
\left.V_{\mathrm{L}}\right|_{t=0}=V_{0}
\end{array},\right.
$$

where $\mathbb{P}$ is the Leray projection onto the divergence free vector fields, without changing $V_{\mathrm{L}}^{4}$. The Fourier multiplier associated to $\mathbb{P}$ has the following form

$$
\mathbb{P}_{n}=1-\frac{1}{|\check{n}|^{2}}\left(\begin{array}{cccc}
\frac{n_{1}^{2}}{a_{1}^{2}} & \frac{n_{1} n_{2}}{a_{1} a_{2}} & \frac{n_{1} n_{3}}{a_{1} a_{3}} & 0 \\
\frac{n_{2} n_{1}}{a_{2} a_{1}} & \frac{n_{2}^{2}}{a_{2}^{2}} & \frac{n_{2} n_{3}}{a_{2} a_{3}} & 0 \\
\frac{n_{3} n_{1}}{a_{3} a_{1}} & \frac{n_{3} n_{2}}{a_{3} a_{2}} & \frac{n_{2}^{3}}{a_{2}^{3}} & 0 \\
0 & 0 & 0 & 0
\end{array}\right),
$$

where $|\check{n}|^{2}=\sum_{j} \frac{n_{j}^{2}}{a_{j}^{2}}$ and 1 is the identity matrix on $\mathbb{C}^{4}$. The operator $\mathcal{A}$ was defined in 1.1 . The solution to the linear equation 3.1 is indeed $V_{\mathrm{L}}(\tau)=e^{-\tau \mathbb{P} \mathcal{A}} V_{0}$. We denote the propagator operator $e^{-\tau \mathbb{P} \mathcal{A}}$ as $\mathcal{L}(\tau)$. One can compute the $\operatorname{matrix} \mathbb{P}_{n} \mathcal{A}$

$$
\mathbb{P}_{n} \mathcal{A}=\left(\begin{array}{cccc}
-\frac{\check{n}_{1} \check{n}_{2}}{|\check{n}|^{2}} & -1+\frac{\check{n}_{1}^{2}}{\check{n}^{2}} & 0 & -\frac{\check{n}_{1} \check{n}_{3}}{F|\check{n}|^{2}} \\
1-\frac{\check{n}_{2}^{2}}{|\check{n}|^{2}} & \frac{\check{n}_{1} \check{n}_{2}}{|\check{n}|^{2}} & 0 & -\frac{\check{n}_{2} \check{n}_{3}}{F|\check{n}|^{2}} \\
-\frac{\check{n}_{2} \check{n}_{3}}{|\check{n}|^{2}} & \frac{\check{n}_{1} \check{n}_{3}}{|\check{n}|^{2}} & 0 & \frac{1}{F}\left(1-\frac{\check{n}_{3}^{2}}{|\check{n}|^{2}}\right) \\
0 & 0 & -\frac{1}{F} & 0
\end{array}\right)
$$

whose eigenvalues are

$$
\omega^{0}(n)=0, \quad i \omega^{ \pm}(n)= \pm \frac{i}{F} \frac{\sqrt{\left|\check{n}_{h}\right|^{2}+F^{2} \check{n}_{3}^{2}}}{|\check{n}|}
$$


where the eigenvalue $\omega^{0}$ has multiplicity 2 , and we can write $\omega^{ \pm}= \pm \omega$. The associated normalized eigenvectors are

$$
e^{0}(n)=\frac{1}{|\check{n}|_{F}}\left(\begin{array}{c}
-\check{n}_{2} \\
\check{n}_{1} \\
0 \\
-F \check{n}_{3}
\end{array}\right), \quad e^{ \pm}(n)=\frac{1}{\left(1+F^{2}|\omega(n)|^{2}\right)\left|\check{n}_{h}\right|^{2}|\check{n}|^{2}}\left(\begin{array}{c}
-F \check{n}_{3}\left(\check{n}_{2} \mp i \check{n}_{1} \omega(n)\right) \\
F \check{n}_{3}\left(\check{n}_{1} \pm i \check{n}_{2} \omega(n)\right) \\
\mp i F \omega(n)\left|\check{n_{h}}\right|^{2} \\
\left|\check{n_{h}}\right|^{2}
\end{array}\right) \text {, }
$$

where $|\check{n}|_{F}=\sqrt{\check{n}_{1}^{2}+\check{n}_{2}^{2}+F^{2} \check{n}_{3}^{2}}$, if $\left|n_{h}\right|, n_{3} \neq 0$, otherwise, respectively

$$
e^{ \pm}\left(0, n_{3}\right)=\frac{1}{\sqrt{2}}\left(\begin{array}{c} 
\pm i \\
1 \\
0 \\
0
\end{array}\right), \quad e^{ \pm}\left(n_{h}, 0\right)=\frac{1}{\sqrt{2}}\left(\begin{array}{c}
0 \\
0 \\
\pm i \\
1
\end{array}\right) .
$$

The eigenvalue $\omega^{0}$ has algebraic multiplicity 2, but there is only one eigenvector related to it, namely $e^{0}$. Indeed the matrix $\mathbb{P} \mathcal{A}$ has a nontrivial $2 \times 2$ Jordan block structure associated to the eigenvalue 0 , hence there is a generalized eigenvector $\tilde{e}^{0}$. This though is not divergence-free, hence it plays no role in the evolution of the system $\left(\mathrm{PE}_{\varepsilon}\right)$, for this reason it is omitted. For a more detailed discussion on the spectral properties of the linear system we refer the reader to the papers 22] and 23.

Once we have introduced the eigenvectors in 3.5 we can consider a generic divergence-free vector field $V$ as direct sum of the elements belonging to $\mathbb{C} e^{0}$ and $\mathbb{C} e^{-} \oplus \mathbb{C} e^{+}$. We denote the projection of $V$ onto $\mathbb{C} e^{0}$ as the quasigeostrophic part of the flow, while we denote the projection onto $\mathbb{C} e^{-} \oplus \mathbb{C} e^{+}$as the oscillating part. The projection can be explicitly defined as follows

$$
V_{\mathrm{QG}}=\mathcal{F}^{-1}\left(\left(\hat{V}_{n} \mid e^{0}(n)\right)_{\mathbb{C}^{4}} e^{0}(n)\right), \quad V_{\mathrm{osc}}=\sum_{i= \pm} \mathcal{F}^{-1}\left(\left(\hat{V}_{n} \mid e^{i}(n)\right)_{\mathbb{C}^{4}} e^{i}(n)\right) .
$$

The element $V_{\text {osc }}$ is called oscillating because is the only part of the initial vector field $V_{0}$ which is affected in the evolution of the system $\left[3.1\right.$, $V_{\mathrm{QG}}$ stays still being in the kernel of $\mathbb{P} \mathcal{A}$.

We would like to point out the following relevant fact, the non-oscillating eigenspace $\mathbb{C} e^{0}$ is orthogonal to the oscillating eigenspace $\mathbb{C} e^{-} \oplus \mathbb{C} e^{+}$, whence in particular it is always true that $V_{\mathrm{QG}} \perp V_{\mathrm{osc}}$.

In the following we shall denote as $e^{a}(n)$ the eigenvector of $\mathbb{P}_{n} \mathcal{A}$ associated with the eigenvalue $i \omega^{a}$, i.e.

$$
e^{\tau \mathbb{P}_{n} \mathcal{A}}\left(e^{i n \cdot x} e^{a}(n)\right)=\exp \left\{i n \cdot x+\tau \omega^{a}(n)\right\} e^{a}(n) .
$$

Let us define $U^{\varepsilon}=\mathcal{L}\left(-\frac{t}{\varepsilon}\right) V^{\varepsilon}$, we want to reformulate $\overline{\mathrm{PE}_{\varepsilon}}$ in terms of the new unknown $U^{\varepsilon}$. A straightforward computation shows that the vector field $U^{\varepsilon}$ satisfies the following equation

$$
\left\{\begin{array}{l}
\partial_{t} U^{\varepsilon}+\mathcal{Q}^{\varepsilon}\left(U^{\varepsilon}, U^{\varepsilon}\right)-\mathbb{D}^{\varepsilon} U^{\varepsilon}=0 \\
\operatorname{div} v^{\varepsilon}=0 \\
\left.U^{\varepsilon}\right|_{t=0}=V_{0}
\end{array}\right.
$$

where

$$
\begin{aligned}
\mathcal{Q}^{\varepsilon}\left(U^{\varepsilon}, U^{\varepsilon}\right) & =\mathcal{L}\left(-\frac{t}{\varepsilon}\right) \mathbb{P}\left[\left(\mathcal{L}\left(\frac{t}{\varepsilon}\right) U^{\varepsilon} \cdot \nabla\right) \mathcal{L}\left(\frac{t}{\varepsilon}\right) U^{\varepsilon}\right], \\
\mathbb{D}^{\varepsilon} U^{\varepsilon} & =\mathcal{L}\left(-\frac{t}{\varepsilon}\right) \mathbf{D} \mathcal{L}\left(\frac{t}{\varepsilon}\right) U^{\varepsilon} .
\end{aligned}
$$

We denote the system $\overline{\mathrm{FS}_{\varepsilon}}$ the filtered system.

Before using the above results to find the limit of $\left[\overline{\left.\mathrm{FS}_{\varepsilon}\right]}\right.$ we introduce the potential vorticity

$$
\Omega^{\varepsilon}=-\partial_{2} U^{1, \varepsilon}+\partial_{1} U^{2, \varepsilon}-F \partial_{3} U^{4, \varepsilon} .
$$

The potential vorticity was introduced by J.-Y. Chemin in $[14$ and it is now a well-known tool in the study of primitive equation (see [12], [13], [24], 29]). The diagonalization explained in 3.7) can as well be obtained by writing $U^{\varepsilon}=$ $U_{\mathrm{QG}}^{\varepsilon}+U_{\mathrm{osc}}^{\varepsilon}$, with

$$
U_{\mathrm{QG}}^{\varepsilon}=\left(\begin{array}{llll}
-\partial_{2} \Delta_{F}^{-1} \Omega^{\varepsilon}, & \partial_{1} \Delta_{F}^{-1} \Omega^{\varepsilon}, & 0, & -\partial_{3} F \Delta_{F}^{-1} \Omega^{\varepsilon}
\end{array}\right),
$$

where $\Delta_{F}^{-1}$ denotes the operator

$$
\left.-\Delta_{F}^{-1} u=\mathcal{F}^{-1}\left(\left(\frac{1}{\check{n}_{1}^{2}+\check{n}_{2}^{2}+F^{2} \check{n}_{3}^{2}} \hat{u}_{n}\right)\right)_{n}\right)
$$


We remark the fact that since $U_{\mathrm{QG}}^{\varepsilon}$ belongs to the kernel of $\mathbb{P} \mathcal{A}$ we can write $U_{\mathrm{QG}}^{\varepsilon}=V_{\mathrm{QG}}^{\varepsilon}$.

One major problem is to understand which is the limit for $\varepsilon \rightarrow 0$ of the forms $\mathcal{Q}^{\varepsilon}, \mathbb{D}^{\varepsilon}$ and, if possible, how to give a closed formulation for it. To do so we use the explicit formulation of $\mathcal{Q}^{\varepsilon}, \mathbb{D}^{\varepsilon}$ given in equation [3.8] and (3.9). Let us decompose the divergence-free vector field $U$ as:

$$
\mathcal{F} U(n)=\sum_{a \in\{-, 0,+\}} U^{a}(n)=\sum_{a \in\{-, 0,+\}}\left(\mathcal{F} U(n) \mid e^{a}(n)\right)_{\mathbb{C}^{4}} e^{a}(n),
$$

and after some computations we obtain that;

$$
\mathcal{F}\left(\mathcal{Q}^{\varepsilon}(U, V)\right)(n)=\sum_{a, b, c \in\{-, 0,+\}} e^{-i \frac{t}{\varepsilon}\left(\omega^{a}(k)+\omega^{b}(n-k)-\omega^{c}(n)\right)}\left(\sum_{j=1,2,3}\left(n_{j}-k_{j}\right) U^{a, j}(k) V^{b}(n-k) \mid e^{c}(n)\right){ }_{\mathbb{C}^{4}} e^{c}(n)
$$

In the following we write $\omega_{k, n-k, n}^{a, b, c}=\omega^{a}(k)+\omega^{b}(n-k)-\omega^{c}(n)$ for the sake of conciseness, as well as $\omega_{n}^{a, b}=\omega^{a}(n)+\omega^{b}(n)$. With $U^{a, j}$ we denote the $j$-th component of the vector $U^{a}=\left(\hat{U} \mid e^{a}\right)_{\mathbb{C}^{4}} e^{a}$ for $a=0, \pm$.

Similar calculations give us that

$$
\mathbb{D}^{\varepsilon} U=\mathcal{F}^{-1}\left(\sum_{a, b \in\{-, 0,+\}} e^{-i \frac{t}{\varepsilon} \omega_{n}^{a, b}}\left(\mathbf{D}(n) U^{b}(n) \mid e^{a}(n)\right)_{\mathbb{C}^{4}} e^{a}(n)\right),
$$

where $\mathbf{D}(n)$ is the Fourier symbol associated to the second-order differential operator $\mathbf{D}$, see $(1.1)$.

Letting $\varepsilon \rightarrow 0$ we only have to use the non stationary phase theorem ( see, for instance [2], |7], [40]) to obtain that, if $U, V$ are smooth functions;

$$
\begin{aligned}
\mathcal{Q}(U, V) & =\mathcal{F}^{-1}\left(\mathbb{P}_{n} \sum_{\omega_{k, n-k, n}^{a, b, c}=0}\left(\sum_{j=1}^{3}\left(n_{j}-k_{j}\right) U^{a, j}(k) V^{b}(n-k) \mid e^{c}(n)\right)_{\mathbb{C}^{4}} e^{c}(n)\right), \\
\mathbb{D} U & =\mathcal{F}^{-1}\left(\sum_{\omega_{n}^{a, b}=0}\left(\mathbf{D}(n) U^{b}(n) \mid e^{a}(n)\right)_{\mathbb{C}^{4}} e^{a}(n)\right) .
\end{aligned}
$$

Here we implicitly define as $\mathbf{D}(n)$ the Fourier symbol associated to the matrix $\mathbf{D}$ defined in 1.1 .

\subsection{The global splitting of the limit bilinear form $\mathcal{Q}$}

This section is aimed to explain how the bilinear interaction $\mathcal{Q}$ defined in 3.14 behaves along the non-oscillating and oscillating subspaces $\mathbb{C} e^{0}$ and $\mathbb{C} e^{-} \oplus \mathbb{C} e^{+}$. Such kind of result is well known in the theory of singular perturbation problems in periodic domains. The results that we present are proved by several authors in [5], [22] and [23], for this reason we do not prove them but instead we refer to the works mentioned and references therein.

In this section we consider smooth vector fields. Given a smooth vector field $W$ we define

$$
\Omega_{W}=-\partial_{2} W^{2}+\partial_{1} W^{2}-F \partial_{3} W^{4}, \quad W_{\mathrm{QG}}=\left(\begin{array}{c}
-\partial_{2} \\
\partial_{1} \\
0 \\
-F \partial_{3}
\end{array}\right) \Delta_{F}^{-1} \Omega_{W}=\left(w_{\mathrm{QG}}, W_{\mathrm{QG}}^{4}\right), \quad W_{\mathrm{osc}}=W-W_{\mathrm{QG}} .
$$

Obviously $W_{\mathrm{QG}}$ and $W_{\mathrm{osc}}$ are respectively the projections of $W$ onto the non-oscillating and oscillating subspaces defined in 3.7 .

Lemma 3.1. The following identity holds true

$$
\mathcal{F}^{-1}\left(\left(\left.\mathcal{F} \mathcal{Q}(W, W)|| n\right|_{F} e^{0}\right)_{\mathbb{C}^{4}}\right)=w_{\mathrm{QG}} \cdot \nabla \Omega_{W},
$$

where $\mathcal{Q}$ is defined in (3.14) and $e^{0}$ is the non-oscillating eigenvector defined in 3.5.

Corollary 3.2. The following identity holds true

$$
\mathcal{F}^{-1}\left(\left(\mathcal{F} \mathcal{Q}(W, W) \mid e^{0}\right)_{\mathbb{C}^{4}} e^{0}\right)=\left(\begin{array}{c}
-\partial_{2} \\
\partial_{1} \\
0 \\
-F \partial_{3}
\end{array}\right) \Delta_{F}^{-1}\left(w_{\mathrm{QG}} \cdot \nabla \Omega_{W}\right)
$$


For a proof of Lemma 3.1 we refer the reader to [22] and 23. What has to be retained is the fact that the projection of $\mathcal{Q}(W, W)$ onto the potential non-oscillating subspace does not present interactions of the oscillating part of the vector field.

Lemma 3.3. Let $W$ be a smooth vector field, then the following identity holds true

$$
\left(\mathcal{Q}\left(W_{\mathrm{QG}}, W_{\mathrm{QG}}\right)\right)_{\mathrm{osc}}=0 \text {. }
$$

Proof. Considering the explicit formulation of the limit bilinear form $\mathcal{Q}$ we deduce

$$
\left(\mathcal{Q}\left(W_{\mathrm{QG}}, W_{\mathrm{QG}}\right)\right)_{\mathrm{osc}}=\mathcal{F}^{-1}\left(\sum_{\substack{k+m=n \\ \omega_{k, m, n}^{0, \pm}=0}}\left(n \cdot\left(W^{0}(k) \otimes W^{0}(m)\right) \mid e^{ \pm}(n)\right)_{\mathbb{C}^{4}} e^{ \pm}(n)\right) .
$$

Let us consider hence the equation $\omega_{k, m, n}^{0,0, \pm}=0$, thanks to the explicit expression of the eigenvalues in [3.4] then it is equivalent to the equation

$$
\left|n_{h}\right|^{2}+F^{2} n_{3}^{2}=0
$$

which is true only if $n=0$, and in this case the contributions arising in 3.16 are null, concluding.

Corollary 3.4. The projection of the limit bilinear form $\mathcal{Q}$ onto the oscillating subspace can be written as

$$
(\mathcal{Q}(W, W))_{\mathrm{osc}}=\left(\mathcal{Q}\left(W_{\mathrm{QG}}, W_{\mathrm{osc}}\right)\right)_{\mathrm{osc}}+\left(\mathcal{Q}\left(W_{\mathrm{osc}}, W_{\mathrm{QG}}\right)\right)_{\mathrm{osc}}+\left(\mathcal{Q}\left(W_{\mathrm{osc}}, W_{\mathrm{osc}}\right)\right)_{\mathrm{osc}} .
$$

\section{Proof of Theorem 1.10}

Remark 4.1. As the reader may have noted Theorem 1.10 states the existence of $\grave{a}$ la Leray-type solutions. This can seem to be unexpected since, generally, Leray solutions are constructed thanks to compactness methods. In system (S) we cannot apply directly any compactness method since we do not have any second-order vertical derivative $\partial_{3}^{2}$ and $H^{1,0}$ is not compactly embedded in $L^{2}$. Nonetheless the bilinear form $\mathcal{Q}$ has better product rules than the standard bilinear form in the Navier-Stokes equations, this allows us to make sense (distributionally) of the term $\mathcal{Q}(U, U)$. Moreover we require the initial potential vorticity $\Omega_{0}$ to be $L^{2}\left(\mathbb{T}^{3}\right)$, which, roughly speaking, is almost as requiring the initial velocity field to be $H^{1}$.

Proof of Theorem 1.10: Before starting the proof we point out the following fact, Navier-Stokes equations preserve the global average of the unknown function. This is true as well for the system $\left[\mathrm{PE}_{\varepsilon}\right]$, whence we can consider data with zero horizontal average. Thanks to this property homogeneous and non-homogeneous Sobolev spaces are equivalent; we use this property constantly in the present proof. This fact concerns the isotropic spaces $H^{s}\left(\mathbb{R}^{3}\right)$ only. The proof is standard application of Galerkin's approximation scheme. We define the truncation operator

$$
J_{n} u=\sum_{\left\{k \in \mathbb{Z}^{3}|| k \mid \leqslant n\right\}} \hat{u}_{n} e^{i \check{k} \cdot x},
$$

and consequently the approximated system

$$
\left\{\begin{array}{l}
\partial_{t} U_{n}+J_{n} \mathcal{Q}\left(U_{n}, U_{n}\right)+\mathbb{D} U_{n}=0 \\
\operatorname{div} u_{n}=0 \\
\left.U_{n}\right|_{t=0}=J_{n} U_{0}
\end{array}\right.
$$

in the unknown $U_{n}$. We recall that for a fixed $n, J_{n}$ maps continuously any $H^{k}$ space to any $H^{k+h}$ space for $h \geqslant 0$ thanks to Bernstein inequality. Thus 4.1 is a differential equation in the space

$$
L_{n}^{2}\left(\mathbb{T}^{3}\right)=\left\{u \in L^{2}\left(\mathbb{T}^{3}\right) \mid \hat{u}_{k}=0 \text { if }|k|>n\right\} .
$$

Since the support of the Fourier transform of $U_{n} \in L_{n}^{2}\left(\mathbb{T}^{3}\right)$ is included in the ball of center 0 and radius $n$ and the support of $\mathcal{F}\left(U_{n} \otimes U_{n}\right)$ is included in $B_{2 n}(0)$ we obtain easily that $J_{n} \mathcal{Q} \in \mathcal{C}\left(L_{n}^{2}\left(\mathbb{T}^{3}\right) \times L_{n}^{2}\left(\mathbb{T}^{3}\right) ; L_{n}^{2}\left(\mathbb{T}^{3}\right)\right)$. Hence CauchyLipschitz theorem gives the existence of a unique solution to 4.1] on a maximal interval of time $\left[0, T_{n}\right.$ ) taking values in $L_{n}^{2}\left(\mathbb{T}^{3}\right)$. Since

$$
\mathcal{Q}(A, B)=\lim _{\varepsilon \rightarrow 0} \mathcal{L}\left(-\frac{t}{\varepsilon}\right) \mathbb{P}\left[\left(\mathcal{L}\left(\frac{t}{\varepsilon}\right) A \cdot \nabla\right) \mathcal{L}\left(\frac{t}{\varepsilon}\right) B\right],
$$


it is clear that $\left(J_{n} \mathcal{Q}\left(U_{n}, U_{n}\right) \mid U_{n}\right)_{L^{2}\left(\mathbb{T}^{3}\right)}=\left(\mathcal{Q}\left(U_{n}, U_{n}\right) \mid U_{n}\right)_{L^{2}\left(\mathbb{T}^{3}\right)}=0$ since div $u_{n}=0$. Hence by a standard energy estimate on the parabolic-hyperbolic equation 4.1 we deduce that

$$
\frac{1}{2}\left\|U_{n}(t)\right\|_{L^{2}\left(\mathbb{T}^{3}\right)}^{2}+c \int_{0}^{t}\left\|\nabla_{h} U_{n}(s)\right\|_{L^{2}\left(\mathbb{T}^{3}\right)}^{2} \mathrm{~d} s \leqslant \frac{1}{2}\left\|U_{0}\right\|_{L^{2}\left(\mathbb{T}^{3}\right)}^{2},
$$

from which for all $t \in\left[0, T_{n}\right)$ we infer that $\left\|U_{n}(t)\right\|_{L^{2}\left(\mathbb{T}^{3}\right)}^{2} \leqslant\left\|J_{n} U_{0}\right\|_{L^{2}\left(\mathbb{T}^{3}\right)}^{2} \leqslant\left\|U_{0}\right\|_{L^{2}\left(\mathbb{T}^{3}\right)}^{2}$. Hence $T_{n}=\infty$ and for all $t>0$, $U_{n}(t)$ satisfies

$$
\left\|U_{n}(t)\right\|_{L^{2}\left(\mathbb{T}^{3}\right)}^{2}+2 c \int_{0}^{t}\left\|\nabla_{h} U_{n}(s)\right\|_{L^{2}\left(\mathbb{T}^{3}\right)}^{2} \mathrm{~d} s \leqslant\left\|U_{0}\right\|_{2}^{2} .
$$

Considered the relation $\left\|U_{n}\right\|_{L^{2}\left((0, t) ; L^{2}\left(\mathbb{T}^{3}\right)\right)} \leqslant \sqrt{t}\left\|U_{n}\right\|_{L^{\infty}\left((0, t) ; L^{2}\left(\mathbb{T}^{3}\right)\right)} \leqslant \sqrt{t}\left\|U_{0}\right\|_{L^{2}\left(\mathbb{T}^{3}\right)}$ we can say that the sequence $U_{n}$ is bounded in $L^{\infty}\left(\mathbb{R}_{+} ; L^{2}\left(\mathbb{T}^{3}\right)\right) \cap L_{\text {loc }}^{2}\left(\mathbb{R}_{+} ; H^{1,0}\right)$. By the structure of 4.1) we obtain easily that $\partial_{t} U_{n}$ is bounded in $L_{\text {loc }}^{2}\left(\mathbb{R}_{+} ; H^{-N}\right)$ for $N$ sufficiently big (the proof of such fact is identical as the proof of Proposition 5.1 , hence $\left(\partial_{t} U_{n}\right)_{n}$ is a sequence of uniformly bounded functions in $L_{\text {loc }}^{2}\left(\mathbb{R}_{+} ; H^{-N}\right)$. We can infer via Aubin-Lions lemma [3] obtaining that $U_{n} \rightarrow U$ in $L_{\text {loc }}^{2}\left(\mathbb{R}_{+} ; H^{-\varepsilon}\left(\mathbb{T}^{3}\right)\right), \varepsilon \in(0, N)$ up to (non-relabeled) subsequences.

Since the sequence $\left(U_{n}\right)_{n}$ converges in $L_{\text {loc }}^{2}\left(\mathbb{R}_{+} ; H^{-\varepsilon}\left(\mathbb{T}^{3}\right)\right)$ only, and products of $H^{-\varepsilon}$ distributions are, a priori, not well defined we introduce a diagonalization method which allows us to split 4.1) in two systems which we are able to handle.

We rely on a diagonalization method introduced introduced by P. Embid and A. Majda in [22. We define

$$
\begin{aligned}
\Omega_{n} & =-\partial_{2} U_{n}^{1}+\partial_{1} U_{n}^{2}-F \partial_{3} U_{n}^{4}, \\
V_{\mathrm{QG}, n}=U_{\mathrm{QG}, n} & =\left(\begin{array}{c}
\nabla_{h}^{\perp} \\
0 \\
-F \partial_{3}
\end{array}\right) \Delta_{F}^{-1} \Omega_{n}, \\
U_{\mathrm{osc}, n} & =U_{n}-U_{\mathrm{QG}, n} .
\end{aligned}
$$

Applying Lemma 3.1 on the smooth vector field $U_{n}$ we deduce that

$$
\left(\left.\mathcal{F} J_{n} \mathcal{Q}\left(U_{n}, U_{n}\right)|| n\right|_{F} e^{0}(n)\right)_{\mathbb{C}^{4}}=\mathcal{F}\left(J_{n}\left(v_{\mathrm{QG}, n}^{h} \cdot \nabla_{h} \Omega_{n}\right)\right) .
$$

Whence the projection of the element $J_{n} \mathcal{Q}\left(U_{n}, U_{n}\right)$ onto the potential space defined by the potential vorticity is the quasi-geostrophic transport $J_{n}\left(v_{\mathrm{QG}, n}^{h} \cdot \nabla_{h} \Omega_{n}\right)$.

Applying Corollary 3.4 we deduce:

$$
\left(J_{n} \mathcal{Q}\left(U_{n}, U_{n}\right)\right)_{\mathrm{osc}}=\left(J_{n} \mathcal{Q}\left(V_{\mathrm{QG}, n}, U_{\mathrm{osc}, n}\right)\right)_{\mathrm{osc}}+\left(J_{n} \mathcal{Q}\left(U_{\mathrm{osc}, n}, V_{\mathrm{QG}, n}\right)\right)_{\mathrm{osc}}+\left(J_{n} \mathcal{Q}\left(U_{\mathrm{osc}, n}, U_{\mathrm{osc}, n}\right)\right)_{\mathrm{osc}} .
$$

Projecting hence 4.1) onto the oscillating subspace and the potential nonoscillating subspace we obtain the following global splitting for the first equation of 4.1 :

$$
\begin{array}{r}
\partial_{t} \Omega_{n}+J_{n}\left(v_{\mathrm{QG}, n}^{h} \cdot \nabla_{h} \Omega_{n}\right)+a_{\mathrm{QG}}\left(D_{h}\right) \Omega_{n}=0 \\
\partial_{t} U_{\mathrm{osc}, n}+\left(J_{n} \mathcal{Q}\left(V_{\mathrm{QG}, n}, U_{\mathrm{osc}, n}\right)\right)_{\mathrm{osc}}+\left(J_{n} \mathcal{Q}\left(U_{\mathrm{osc}, n}, V_{\mathrm{QG}, n}\right)\right)_{\mathrm{osc}} \\
+\left(J_{n} \mathcal{Q}\left(U_{\mathrm{osc}, n}, U_{\mathrm{osc}, n}\right)\right)_{\mathrm{osc}}+a_{\mathrm{osc}}\left(D_{h}\right) U_{\mathrm{osc}, n}=0 .
\end{array}
$$

The operators $a_{\mathrm{QG}}$ and $a_{\mathrm{osc}}$ are nothing but the projection of the operator $-\mathbb{D}$ onto the potential space defined by $\Omega$ and the oscillating subspace. We avoid to give a detailed description of such operators now (see Section 5 , what has to be retained is that they are symbols such that there exists a positive constant $c$ such that $a_{\mathrm{QG}}\left(\check{n}_{)}, a_{\mathrm{osc}}(\check{n}) \geq c\left|\check{n}_{h}\right|^{2}\right.$. On the splitting 4.4 we can apply the same procedure as above to obtain that $\Omega_{n} \rightarrow \Omega$ in $L_{\text {loc }}^{2}\left(\mathbb{R}_{+} ; H^{-\varepsilon}\left(\mathbb{T}^{3}\right)\right)$, and defining $V_{\mathrm{QG}}=\left(\begin{array}{c}\nabla_{h}^{\perp} \\ 0 \\ -F \partial_{3}\end{array}\right) \Delta_{F}^{-1} \Omega$ where $\Omega$ is the limit of the sequence $\left(\Omega_{n}\right)_{n}$, and since

$$
\left(\begin{array}{c}
\nabla_{h}^{\perp} \\
0 \\
-F \partial_{3}
\end{array}\right) \Delta_{F}^{-1} \in \mathcal{L}\left(H^{\alpha}, H^{\alpha+1}\right), \alpha \in \mathbb{R}
$$

we obtain as well that

$$
V_{\mathrm{QG}, n} \rightarrow V_{\mathrm{QG}} \text { in } L_{\mathrm{loc}}^{2}\left(\mathbb{R}_{+} ; H^{1-\varepsilon}\right),
$$

and $\left(V_{\mathrm{QG}, n}\right)_{n}$ uniformly (in $n$ ) bounded in $L^{\infty}\left(\mathbb{R}_{+}, H^{1}\right)$. 
Combining the definitions 4.2 and 4.3 we can hence rewrite $V_{\mathrm{QG}, n}$ as

$$
V_{\mathrm{QG}, n}=\left(\begin{array}{c}
-\partial_{2} \\
\partial_{1} \\
0 \\
-F \partial_{3}
\end{array}\right) \Delta_{F}^{-1}\left(\begin{array}{lll}
-\partial_{2}, & \partial_{1}, & 0,-F \partial_{3}
\end{array}\right) \cdot U_{n}=\Pi_{\mathrm{QG}} U_{n},
$$

with $\Pi_{\mathrm{QG}}$ Fourier multiplier of order zero, hence $\Pi_{\mathrm{QG}} \in \mathcal{L}\left(H^{\alpha}\left(\mathbb{T}^{3}\right)\right)$ for each $\alpha \in \mathbb{R}$. This implies that, since $U_{\mathrm{osc}}=$ $U-V_{\mathrm{QG}}$

$$
\begin{aligned}
\left\|U_{\mathrm{osc}, n}-U_{\mathrm{osc}}\right\|_{H^{-\varepsilon}} & =\left\|\left(U_{n}-V_{\mathrm{QG}, n}\right)-\left(U-V_{\mathrm{QG}}\right)\right\| \\
& =\left\|\left(1-\Pi_{\mathrm{QG}}\right)\left(U_{n}-U\right)\right\|_{H^{-\varepsilon}} \\
& \leqslant C\left\|U_{n}-U\right\|_{H^{-\varepsilon}} .
\end{aligned}
$$

We deduce hence that $U_{\mathrm{osc}, n} \rightarrow U_{\mathrm{osc}}$ in $L_{\text {loc }}^{2}\left(\mathbb{R}_{+} ; H^{-\varepsilon}\left(\mathbb{T}^{3}\right)\right)$. The same idea can be applied to show that $\left(U_{\mathrm{osc}, n}\right)_{n}$ is bounded in $L^{\infty}\left(\mathbb{R}_{+} ; L^{2}\left(\mathbb{T}^{3}\right)\right)$ and $\left(\nabla_{h} U_{\mathrm{osc}, n}\right)_{n}$ is bounded in $L^{2}\left(\mathbb{R}_{+} ; L^{2}\left(\mathbb{T}^{3}\right)\right)$.

At this point we can project $\mathcal{Q}\left(U_{n}, U_{n}\right)$ on the spaces $\mathbb{C} e^{0}, \mathbb{C} e^{-} \oplus \mathbb{C} e^{+}$(see [3.5) obtaining, thanks to the results of Corollary 3.2 and 3.4

$$
\begin{aligned}
\mathcal{Q}\left(U_{n}, U_{n}\right)= & \mathcal{Q}\left(U_{n}, U_{n}\right)_{\mathrm{QG}}+\mathcal{Q}\left(U_{n}, U_{n}\right)_{\mathrm{osc}} \\
= & \left(-\partial_{2}, \partial_{1}, 0,-F \partial_{3}\right)^{\top} \Delta_{F}^{-1}\left(v_{\mathrm{QG}, n}^{h} \cdot \nabla_{h} \Omega_{n}\right) \\
& +\left(\mathcal{Q}\left(V_{\mathrm{QG}, n}, U_{\mathrm{osc}, n}\right)\right)_{\mathrm{osc}}+\left(\mathcal{Q}\left(U_{\mathrm{osc}, n}, V_{\mathrm{QG}, n}\right)\right)_{\mathrm{osc}}+\left(\mathcal{Q}\left(U_{\mathrm{osc}, n}, U_{\mathrm{osc}, n}\right)\right)_{\mathrm{osc}} .
\end{aligned}
$$

It is matter of standard energy bounds with classical product rules in Sobolev spaces to prove that

$$
\begin{aligned}
&\left(-\partial_{2}, \partial_{1}, 0,-F \partial_{3}\right)^{\top} \Delta_{F}^{-1}\left(v_{\mathrm{QG}, n}^{h} \cdot \nabla_{h} \Omega_{n}\right) \rightarrow\left(-\partial_{2}, \partial_{1}, 0,-F \partial_{3}\right)^{\top} \Delta_{F}^{-1}\left(v_{\mathrm{QG}}^{h} \cdot \nabla_{h} \Omega\right), \\
&\left(\mathcal{Q}\left(V_{\mathrm{QG}, n}, U_{\mathrm{osc}, n}\right)\right)_{\mathrm{osc}} \rightarrow\left(\mathcal{Q}\left(V_{\mathrm{QG}}, U_{\mathrm{osc}}\right)\right)_{\mathrm{osc}}, \\
&\left(\mathcal{Q}\left(U_{\mathrm{osc}, n}, V_{\mathrm{QG}, n}\right)\right)_{\mathrm{osc}} \rightarrow\left(\mathcal{Q}\left(U_{\mathrm{osc}}, V_{\mathrm{QG}}\right)\right)_{\mathrm{osc}},
\end{aligned}
$$

in the sense of distributions as $n \rightarrow \infty$. The limit of the product of terms of the form $U_{\mathrm{osc}, n}$ is, in general, not well defined. Indeed system [S] lacks of vertical dissipation, hence the best we know is that $U_{\mathrm{osc}, n} \rightarrow U_{\mathrm{osc}}$ in $L_{\mathrm{loc}}^{2}\left(\mathbb{R}_{+} ; H^{-\varepsilon}\right)$, but generally a product between $H^{-\varepsilon}$ elements is not well-defined. Is in this context in fact that we shall use the improved regularity in the product which is characteristic of the bilinear form $\mathcal{Q}$. We claim that

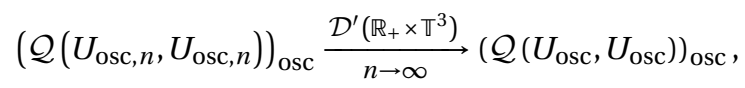

The proof of 4.5 is postponed. Whence we finally proved that $\mathcal{Q}\left(U_{n}, U_{n}\right) \rightarrow \mathcal{Q}(U, U)$ in $\mathcal{D}^{\prime}\left(\mathbb{R}_{+} \times \mathbb{T}^{3}\right)$, concluding.

\subsection{Proof of (4.5)}

As we already stated M. Paicu in [35 proved a similar result. We prove 4.5] using different techniques. Defining $\mathcal{Q}(A, B)=\operatorname{div} \widetilde{\mathcal{Q}}(A, B)$, i.e.

$$
\begin{aligned}
\widetilde{\mathcal{Q}}(A, B) & =\sum_{\mathcal{K}}\left(\hat{A}^{a}(k) \hat{B}^{b}(m) \mid e^{c}(n)\right)_{\mathbb{C}^{4}} e^{c}(n), \\
& =\sum_{\mathcal{K}} \hat{A}^{a}(k) \hat{B}^{b, c}(m, n)
\end{aligned}
$$

where $\hat{A}^{a}(k)=\left(\hat{A}(k) \mid e^{a}(k)\right) e^{a}(k), \hat{B}^{b, c}(m, n)=\left(\hat{B}^{b}(m) \mid e^{c}(n)\right) e^{c}(n)$. It suffice in fact to prove that

$$
\widetilde{\mathcal{Q}}\left(U_{\mathrm{osc}, j}-U_{\mathrm{osc}}, U_{\mathrm{osc}, j}+U_{\mathrm{osc}}\right) \rightarrow 0,
$$

in $\mathcal{D}^{\prime}\left(\mathbb{R}_{+} \times \mathbb{T}^{3}\right)$ as $j \rightarrow \infty$ to conclude. To do so we consider a $\phi \in \mathcal{D}$ and, by Plancherel theorem

$$
\begin{aligned}
\int_{\mathbb{R}_{+} \times \mathbb{T}^{3}} \phi & (t, x) \widetilde{\mathcal{Q}}\left(U_{\mathrm{osc}, j}-U_{\mathrm{osc}}, U_{\mathrm{osc}, j}+U_{\mathrm{osc}}\right)(t, x) \mathrm{d} x \mathrm{~d} t \\
= & \int_{\mathbb{R}_{+}} \sum_{n \in \mathbb{Z}^{3}} \sum_{\mathcal{K}_{n}^{\star}} \hat{\phi}(t, n)\left(\widehat{U_{\mathrm{osc}, j}-U_{\mathrm{osc}}}\right)^{a}(t, k)\left(\widehat{U_{\mathrm{osc}, j}+U_{\mathrm{osc}}}\right)^{b, c}(t, m, n) \mathrm{d} t \\
& =\int_{\mathbb{R}_{+}} \sum_{n, k_{h}, m_{h}} \hat{\phi}_{n}(t) \sum_{\left\{k_{3}:\left(k,\left(m_{h}, n_{3}-k_{3}\right), n\right) \in \mathcal{K}^{\star}\right\}}\left(\widehat{U_{\mathrm{osc}, j-U_{\mathrm{osc}}}}\right)^{a}(t, k) \widehat{\left(U_{\mathrm{osc}, j+U_{\mathrm{osc}}}\right)^{b, c}\left(t, m_{h}, n_{3}-k_{3}, n\right) \mathrm{d} t}
\end{aligned}
$$


We make a couple of remarks in order to simplify the notation. Since we considered the eigenvectors as normalized the following relations are easy to deduce

$$
\left|\hat{U}^{b, c}(m, n)\right| \lesssim\left|\hat{U}^{b}(m)\right| \lesssim|\hat{U}(m)|
$$

Hence from now on the terms $\left(\widehat{U}_{\mathrm{osc}, j-U_{\mathrm{osc}}}\right)^{a}(t, k)$ and $\left(\widehat{U}_{\mathrm{osc}, j+U_{\mathrm{osc}}}\right)^{b, c}\left(t, m_{h}, n_{3}-k_{3}, n\right)$ shall be substituted respectively with $\left(U_{\mathrm{osc}, j}-U_{\mathrm{osc}}\right)_{k}$ and $\left(U_{\mathrm{osc}, j+U_{\mathrm{osc}}}\right)_{\left(m_{h}, n_{3}-k_{3}\right)}$. Here we chose to make implicit the dependence on the variable $t$. We want to stress out the fact that this choice is made only to simplify the notation. Indeed we have that

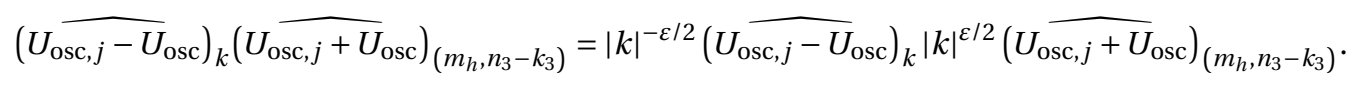

The set $\left\{k_{3}:(n, k) \in \mathcal{K}^{\star}\right\}$ is indeed finite and, in particular, it is composed of the $k_{3}$ which satisfy the following equation

$$
\begin{aligned}
& \left(F^{2}\left(k_{3}\right)^{2}+\left(k_{h}\right)^{2}\right)^{1 / 2}\left(\left(m_{h}\right)^{2}+\left(n_{3}-k_{3}\right)^{2}\right)^{1 / 2} \\
& \quad=\left(\left(k_{h}\right)^{2}+\left(k_{3}\right)^{2}\right)^{1 / 2}\left(\left(m_{h}\right)^{2}+\left(n_{3}-k_{3}\right)^{2}\right)^{1 / 2}-\left(\left(k_{h}\right)^{2}+\left(k_{3}\right)^{2}\right)^{1 / 2}\left(F^{2}\left(n_{3}-k_{3}\right)^{2}+\left(m_{h}\right)^{2}\right)^{1 / 2}
\end{aligned}
$$

Expanding the above equation and collecting term by term in the powers of $k_{3}$, we deduce the following polynomial equation

$$
\wp\left(k_{3}\right)=\sum_{i=0}^{8} A_{i}\left(k_{h}, m_{h}, n\right) k_{3}^{i}=0,
$$

where the $A_{i}$ take the following form

$$
\begin{aligned}
& A_{8}=\left(1-4 F^{2}\right) \\
& A_{7}=4\left(-1+4 F^{2}\right) n_{3} \\
& A_{6}=-6\left(F^{2} k_{h}^{2}+F^{2} m_{h}^{2}+\left(-1+4 F^{2}\right) n_{3}^{2}\right) \\
& A_{5}=4 n_{3}\left(6 F^{2} k_{h}^{2}+3 F^{2} m_{h}^{2}+\left(-1+4 F^{2}\right) n_{3}^{2}\right) \\
& A_{4}=-\left(F^{2}\left(-4+F^{2}\right) k_{h}^{4}+F^{2}\left(-4+F^{2}\right) m_{h}^{4}-6 F^{2} m_{h}^{2} n_{3}^{2}+\left(1-4 F^{2}\right) n_{3}^{4}-2 k_{h}^{2}\left(\left(3+2 F^{2}+F^{4}\right) m_{h}^{2}+18 F^{2} n_{3}^{2}\right)\right) \\
& A_{3}=4 k_{h}^{2} n_{3}\left(-F^{2}\left(-4+F^{2}\right) k_{h}^{2}+\left(3+2 F^{2}+F^{4}\right) m_{h}^{2}+6 F^{2} n_{3}^{2}\right) \\
& A_{2}=-2 k_{h}^{2}\left(\left(2+F^{2}\right) m_{h}^{4}+\left(3+2 F^{2}+F^{4}\right) m_{h}^{2} n_{3}^{2}+3 F^{2} n_{3}^{4}+k_{h}^{2}\left(\left(2+F^{2}\right) m_{h}^{2}-3 F^{2}\left(-4+F^{2}\right) n_{3}^{2}\right)\right) \\
& A_{1}=4 k_{h}^{4} n_{3}\left(\left(2+F^{2}\right) m_{h}^{2}-F^{2}\left(-4+F^{2}\right) n_{3}^{2}\right) \\
& A_{0}=-k_{h}^{4}\left(3 m_{h}^{4}+2\left(2+F^{2}\right) m_{h}^{2} n_{3}^{2}-F^{2}\left(-4+F^{2}\right) n_{3}^{4}\right) .
\end{aligned}
$$

Although we give the explicit expression of the $A_{i}$ 's we underline the fact that the explicit expression is by itself irrelevant. The only thing that matters is that the $A_{i}$ 's are polynomials in the variables $k_{h}, m_{h}, n$. We can hence apply the following result which bounds the modulus of of a complex root of a polynomial in terms of its coefficients. The following proposition is known as Fujiwara near-optimal bound.

Proposition 4.2. Let $P(z)=\sum_{k=0}^{n} a_{n} z^{k}$ a polynomial $P \in \mathbb{C}[z]$, and let $\zeta$ be one of the $n$ complex roots of $P$, then

$$
|\zeta| \leqslant 2 \max \left\{\left|\frac{a_{n-1}}{a_{n}}\right|,\left|\frac{a_{n-2}}{a_{n}}\right|^{1 / 2}, \ldots,\left|\frac{a_{1}}{a_{n}}\right|^{1 /(n-1)},\left|\frac{a_{0}}{a_{n}}\right|^{1 / n}\right\} .
$$

We omit to prove Proposition 4.2 and refer the reader to the work 33 instead. Proposition 4.2 applied on $\wp\left(k_{3}\right)$ tells us that

$$
\left|k_{3}\right| \lesssim|n|^{\alpha_{1}}\left|m_{h}\right|^{\alpha_{2}}\left|k_{h}\right|^{\alpha_{3}}
$$

where $k_{3}$ is any root of $\wp$. Hence

$$
|k|^{\varepsilon / 2} \lesssim\left|k_{h}\right|^{\varepsilon / 2}+\left(|n|^{\alpha_{1}}\left|m_{h}\right|^{\alpha_{2}}\left|k_{h}\right|^{\alpha_{3}}\right)^{\varepsilon / 2},
$$

by concavity on the function $h_{\varepsilon}(x)=x^{\varepsilon / 2}$, with $\alpha_{1}+\alpha_{2}+\alpha_{3}<N$ for some large and finite $N$. Coming back to 4.6] and 
(4.7) this means that

$$
\begin{aligned}
& \left|\int_{\mathbb{R}_{+}} \int_{\mathbb{T}^{3}} \phi(x) \widetilde{\mathcal{Q}}\left(U_{\mathrm{osc}, j}-U_{\mathrm{osc}}, U_{\mathrm{osc}, j}+U_{\mathrm{osc}}\right)(x) \mathrm{d} x \mathrm{~d} t\right| \\
& \lesssim \int_{\mathbb{R}_{+}} \sum_{n, k_{h}, m_{h}}\left|\hat{\phi}_{n}\right| \sum_{\left\{k_{3}:\left(k,\left(m_{h}, n_{3}-k_{3}\right), n\right) \in \mathcal{K}^{\star}\right\}}\left|k_{h}\right|^{\varepsilon / 2}|k|^{-\varepsilon / 2}\left|\left(U_{\mathrm{osc}, j}-U_{\mathrm{osc}}\right)_{k}\right| \\
& \times\left|\left(U_{\mathrm{osc}, j}+U_{\mathrm{osc}}\right)_{\left(m_{h}, n_{3}-k_{3}\right)}\right| \mathrm{d} t \\
& +\int_{\mathbb{R}_{+}} \sum_{n, k_{h}, m_{h}}\left|\hat{\phi}_{n}\right| \sum_{\left\{k_{3}:\left(k,\left(m_{h}, n_{3}-k_{3}\right), n\right) \in \mathcal{K}^{\star}\right\}}|k|^{-\varepsilon / 2}\left|\left(\overline{U_{\mathrm{osc}, j}-U_{\mathrm{osc}}}\right)_{k}\right| \\
& \times\left(|n|^{\alpha_{1}}\left|m_{h}\right|^{\alpha_{2}}\left|k_{h}\right|^{\alpha_{3}}\right)^{\varepsilon / 2}\left|\left(U_{\mathrm{osc}, j+U_{\mathrm{osc}}}\right)_{\left(m_{h}, n_{3}-k_{3}\right)}\right| \mathrm{d} t \\
& =\int_{\mathbb{R}_{+}} \sum_{n, k_{h}, m_{h}}\left|\hat{\phi}_{n}\right| \sum_{\left\{k_{3}:\left(k,\left(m_{h}, n_{3}-k_{3}\right), n\right) \in \mathcal{K}^{\star}\right\}}\left|k_{h}\right|^{\varepsilon / 2}|k|^{-\varepsilon / 2}\left|\left(U_{\mathrm{osc}, j}-U_{\mathrm{osc}}\right)_{k}\right| \\
& \times\left|\left(U_{\mathrm{osc}, j}+U_{\mathrm{osc}}\right)_{\left(m_{h}, n_{3}-k_{3}\right)}\right| \mathrm{d} t \\
& +\int_{\mathbb{R}_{+}} \sum_{n, k_{h}, m_{h}}|n|^{\frac{\alpha_{1} \varepsilon}{2}}\left|\hat{\phi}_{n}\right| \sum_{\left\{k_{3}:\left(k,\left(m_{h}, n_{3}-k_{3}\right), n\right) \in \mathcal{K}^{\star}\right\}}\left|k_{h}\right|^{\frac{\alpha_{3} \varepsilon}{2}}|k|^{-\varepsilon / 2}\left|\left(U_{\mathrm{osc}, j-U_{\mathrm{osc}}}\right)_{k}\right| \\
& \times\left|m_{h}\right|^{\frac{\alpha_{2} \varepsilon}{2}}\left|\left(U_{\mathrm{osc}, j}+U_{\mathrm{osc}}\right)_{\left(m_{h}, n_{3}-k_{3}\right)}\right| \mathrm{d} t \\
& =I_{1, j}+I_{2, j} \text {. }
\end{aligned}
$$

We prove that $I_{2, j} \rightarrow 0$ as $j \rightarrow \infty$. In order to prove that $I_{1, j} \rightarrow 0$ the procedure is very similar (and actually simpler) to the one we are going to perform now, for this reason is omitted. We start remarking that

$$
\left|k_{h}\right|^{\frac{\alpha_{3} \varepsilon}{2}}|k|^{-\varepsilon / 2}\left|\left(U_{\mathrm{osc}, j-U_{\mathrm{osc}}}\right)_{k}\right|=\left(|k|^{-\varepsilon}\left|\left(\widehat{U_{\mathrm{osc}, j}-U_{\mathrm{osc}}}\right)_{k}\right|\right)^{1 / 2}\left(\left|k_{h}\right|^{\alpha_{3} \varepsilon}\left|\left(\widehat{U_{\mathrm{osc}, j}-U_{\mathrm{osc}}}\right)_{k}\right|\right)^{1 / 2},
$$

hence

$$
\begin{aligned}
& \left.I_{2, j} \lesssim \int_{\mathbb{R}_{+}} \sum_{n, k_{h}, m_{h}}|n|^{\frac{\alpha_{1} \varepsilon}{2}}\left|\hat{\phi}_{n}\right| \sum_{\left\{k_{3}:\left(k,\left(m_{h}, n_{3}-k_{3}\right), n\right) \in \mathcal{K}^{\star}\right\}}\left(|k|^{-\varepsilon}\left|\left(U_{\mathrm{osc}, j}-U_{\mathrm{osc}}\right)_{k}\right|\right)\right)^{1 / 2} \\
& \times\left(\left|k_{h}\right|^{\alpha_{3} \varepsilon}\left|\left(U_{\mathrm{osc}, j-U_{\mathrm{osc}}}\right)_{k}\right|\right)^{1 / 2}\left|m_{h}\right|^{\frac{\alpha_{2} \varepsilon}{2}}\left|\left(U_{\mathrm{osc}, j+U_{\mathrm{osc}}}\right)_{\left(m_{h}, n_{3}-k_{3}\right)}\right| \mathrm{d} t .
\end{aligned}
$$

Applying Lemma 8.4 we obtain

$$
I_{2, j} \lesssim\|\phi\|_{L_{\mathrm{loc}}^{\infty}\left(\mathbb{R}_{+} ; H^{\frac{1}{2}+\frac{\alpha_{1} \varepsilon}{2}}\right)}\left\|U_{\mathrm{osc}, j}+U_{\mathrm{osc}}\right\|_{L_{\mathrm{loc}}^{2}\left(\mathbb{R}_{+} ; H^{\frac{1}{2}+\frac{\alpha_{2} \varepsilon}{2}, 0}\right)}\left\|U_{\mathrm{osc}, j}-U_{\mathrm{osc}}\right\|_{L_{\mathrm{loc}}^{2}\left(\mathbb{R}_{+} ; H^{\alpha_{3} \varepsilon, 0}\right)}^{1 / 2}\left\|U_{\mathrm{osc}, j}-U_{\mathrm{osc}}\right\|_{L_{\mathrm{loc}}^{\infty}\left(\mathbb{R}_{+} ; H^{-\varepsilon}\right)}^{1 / 2} .
$$

Both $U_{\text {osc }, j}, U_{\text {osc }}$ belong to $L^{\infty}\left(\mathbb{R}_{+} ; L^{2}\right)$ and $L^{2}\left(\mathbb{R}_{+} ; \dot{H}^{1,0}\right)$, and hence to $L_{\text {loc }}^{2}\left(\mathbb{R}_{+} ; L^{2}\right)$ and by interpolation to $L_{\text {loc }}^{2}\left(\mathbb{R}_{+} ; \dot{H}^{\sigma, 0}\right)$ for $\sigma \in(0,1)$. This means that is $\varepsilon$ is sufficiently small the quantities $\left\|U_{\mathrm{osc}, j}+U_{\mathrm{osc}}\right\|_{L_{\mathrm{loc}}^{2}\left(\mathbb{R}_{+} ; H^{\frac{1}{2}+\frac{\alpha_{2} \varepsilon}{2}, 0}\right)},\left\|U_{\mathrm{osc}, j}-U_{\mathrm{osc}}\right\|_{L_{\mathrm{loc}}^{2}\left(\mathbb{R}_{+} ; H^{\alpha_{3} \varepsilon, 0}\right)}^{2}$ are bounded, while since

$$
\left\|U_{\mathrm{osc}, j}-U_{\mathrm{osc}}\right\|_{L_{\mathrm{loc}}^{\infty}\left(\mathbb{R}_{+} ; H^{-\varepsilon}\right)}^{2} \stackrel{j \rightarrow \infty}{\longrightarrow} 0,
$$

we proved that $I_{2, j} \rightarrow 0$ distributionally. This implies hence that $\mathcal{Q}\left(U_{\mathrm{osc}, j}, U_{\mathrm{osc}, j}\right) \rightarrow \mathcal{Q}\left(U_{\mathrm{osc}}, U_{\mathrm{osc}}\right)$ in a distributional sense.

\section{Weak convergence as $\varepsilon \rightarrow 0$}

In the present section we prove Theorem 1.11

It is natural to ask if, in the limit $\varepsilon \rightarrow 0$, the filtered system $\left[\mathrm{FS}_{\varepsilon}\right]$ converges to the limit system $[\mathrm{S}]$.

Proposition 5.1. Let $U_{0} \in H^{0, s}$ and $U^{\varepsilon}$ be a local strong solution identified by Theorem 1.4 of $\left[\overline{\mathrm{FS}_{\varepsilon}}\right]$, then the sequence $\left(U^{\varepsilon}\right)_{\varepsilon>0}$ has the following regularity uniformly in $\varepsilon$

$$
U^{\varepsilon} \in L^{\infty}\left(\mathbb{R}_{+} ; L^{2}\left(\mathbb{T}^{3}\right)\right), \quad \nabla_{h} U^{\varepsilon} \in L^{2}\left(\mathbb{R}_{+} ; L^{2}\left(\mathbb{T}^{3}\right)\right),
$$

and is compact in the space

$$
L_{\text {loc }}^{2}\left(\mathbb{R}_{+} ; H^{-\eta}\left(\mathbb{T}^{3}\right)\right),
$$

for some $\eta>0$ (possibly small). 
Proof. The proof of 5.1 is merely an $L^{2}\left(\mathbb{T}^{3}\right)$ energy estimate on the filtered system $\overline{\mathrm{FS}_{\varepsilon}}$, hence is omitted. We prove now that $\left(\partial_{t} U^{\varepsilon}\right)_{\varepsilon}$ is bounded, uniformly in $\varepsilon$, in $L_{\text {loc }}^{2}\left(\mathbb{R}_{+} ; H^{-N}\right)$ where $N$ is large.

The only thing to prove is to control the bilinear interaction $\mathcal{Q}^{\varepsilon}\left(U^{\varepsilon}, U^{\varepsilon}\right)$ in the $L_{\text {loc }}^{2}\left(\mathbb{R}_{+} ; H^{-N}\right)$ space. Let $\phi$ be a test function:

$$
\begin{aligned}
\left|\int_{\mathbb{R}_{+} \times \mathbb{T}^{3}} \mathcal{Q}^{\varepsilon}\left(U^{\varepsilon}, U^{\varepsilon}\right) \cdot \phi \mathrm{d} x \mathrm{~d} t\right| & =\left|\int_{\mathbb{R}_{+} \times \mathbb{T}^{3}}\left[\mathcal{L}\left(\frac{t}{\varepsilon}\right) U^{\varepsilon} \otimes \mathcal{L}\left(\frac{t}{\varepsilon}\right) U^{\varepsilon}\right]: \nabla \phi \mathrm{d} x \mathrm{~d} t\right| \\
& \leqslant\left\|U^{\varepsilon}\right\|_{L^{\infty}\left(\mathbb{R}_{+} ; L^{2}\right)}\left\|U^{\varepsilon}\right\|_{L_{\mathrm{loc}}^{2}\left(\mathbb{R}_{+} ; H^{1,0}\right)}\|\nabla \phi\|_{L^{2}\left(\mathbb{R}_{+} ; L_{\nu}^{\infty} L_{h}^{2}\right)}
\end{aligned}
$$

Indeed [5.1) assures us that $U^{\varepsilon} \in L_{\text {loc }}^{2}\left(\mathbb{R}_{+} ; H^{1,0}\left(\mathbb{T}^{3}\right)\right)$ uniformly in $\varepsilon$, whence, by density, we proved that $\left(\partial_{t} U^{\varepsilon}\right)_{\varepsilon}$ is bounded, uniformly in $\varepsilon$, in $L_{\text {loc }}^{2}\left(\mathbb{R}_{+} ; H^{-N}\right)$ where $N$ is large. It suffice hence to apply Aubin-Lions lemma (see [3|) to deduce the claim.

Proposition 5.1 asserts hence that (up to subsequences, not relabeled):

$$
U^{\varepsilon}=U+r^{\varepsilon},
$$

where $r^{\varepsilon}$ is an $L_{\text {loc }}^{2}\left(\mathbb{R}_{+} ; H^{-\eta}\left(\mathbb{T}^{3}\right)\right)$ perturbation and $U$ is a non-highly-oscillating state. In what follows we denote as $V_{\mathrm{QG}}$ the projection onto the non-oscillating space defined in (3.7) of the limit non-highly-oscillating state $U$, similarly $U_{\text {osc }}$ is the projection of $U$ onto the oscillating subspace. The element $\Omega$ is indeed defined as $\Omega=-\partial_{2} U^{1}+\partial_{1} U^{2}-$ $\mathrm{Fo}_{3} U^{4}$.

First of all we have to make sense of a convergence of the from

$$
\mathcal{Q}^{\varepsilon}\left(U^{\varepsilon}, U^{\varepsilon}\right) \rightarrow \mathcal{Q}(U, U),
$$

where $U$ is a weak solution of the limit system $[\mathrm{S}]$ of which we can say at best that it belongs to the space

$$
U \in L^{\infty}\left(\mathbb{R}_{+} ; L^{2}\left(\mathbb{T}^{3}\right)\right), \quad \nabla_{h} U \in L^{2}\left(\mathbb{R}_{+} ; L^{2}\left(\mathbb{T}^{3}\right)\right),
$$

thanks to Theorem $1.10\left(U^{\varepsilon}\right)_{\varepsilon>0}$ a (not relabeled) sequence of local strong solutions of $\left[\mathrm{FS}_{\varepsilon}\right.$ which satisfy [5.1] uniformly in $\varepsilon$ and that converges to a limit element $U$ in $L_{\text {loc }}^{2}\left(\mathbb{R}_{+} ; H^{-\eta}\right)$ for some $\eta>0$. In fact, in order to define $\mathcal{Q}$ in [3.14, we applied the nonstationary phase theorem for smooth function. This is obviously not the case, but mollifying the data it is possible to deduce an analogous result;

Lemma 5.2. Let $\left(U^{\varepsilon}\right)_{\varepsilon>0}$ a (not relabeled) sequence of local strong solutions of $\left[\mathrm{FS}_{\varepsilon}\right.$ which satisfy [5.1] uniformly in $\varepsilon$ and that converges to a limit element $U$ in $L_{\text {loc }}^{2}\left(\mathbb{R}_{+} ; H^{-\eta}\right)$ for some $\eta>0$. Then the following limit holds in the sense of distributions

$$
\mathcal{Q}^{\varepsilon}\left(U^{\varepsilon}, U^{\varepsilon}\right) \rightarrow \mathcal{Q}(U, U)
$$

Proof. Let us define the mollifications

$$
U_{\alpha}^{\varepsilon}=\mathcal{F}^{-1}\left(1_{\left\{|n| \leqslant \frac{1}{\alpha}\right\}} \hat{U}^{\varepsilon}\right), \quad \quad U_{\alpha}=\mathcal{F}^{-1}\left(1_{\left\{|n| \leqslant \frac{1}{\alpha}\right\}} \hat{U}\right) .
$$

Indeed

and

$$
\begin{aligned}
\mathcal{Q}^{\varepsilon}\left(U^{\varepsilon}, U^{\varepsilon}\right)-\mathcal{Q}(U, U)= & \mathcal{Q}^{\varepsilon}\left(U^{\varepsilon}, U^{\varepsilon}\right)-\mathcal{Q}^{\varepsilon}\left(U_{\alpha}^{\varepsilon}, U_{\alpha}^{\varepsilon}\right) \\
& +\mathcal{Q}^{\varepsilon}\left(U_{\alpha}^{\varepsilon}, U_{\alpha}^{\varepsilon}\right)-\mathcal{Q}\left(U_{\alpha}, U_{\alpha}\right) \\
& +\mathcal{Q}\left(U_{\alpha}, U_{\alpha}\right)-\mathcal{Q}(U, U),
\end{aligned}
$$

$$
\begin{gathered}
\mathcal{Q}^{\varepsilon}\left(U^{\varepsilon}, U^{\varepsilon}\right)-\mathcal{Q}^{\varepsilon}\left(U_{\alpha}^{\varepsilon}, U_{\alpha}^{\varepsilon}\right) \stackrel{\alpha \rightarrow 0}{\longrightarrow} 0, \\
\mathcal{Q}\left(U_{\alpha}, U_{\alpha}\right)-\mathcal{Q}(U, U) \stackrel{\alpha \rightarrow 0}{\longrightarrow} 0,
\end{gathered}
$$

weakly since $U_{\alpha}^{\varepsilon} \stackrel{\alpha \rightarrow 0}{\longrightarrow} U^{\varepsilon}, U_{\alpha} \stackrel{\alpha \rightarrow 0}{\longrightarrow} U$ in $L_{\text {loc }}^{\infty}\left(\mathbb{R}_{+} ; L^{2}\left(\mathbb{T}^{3}\right)\right)$. Being the space domain $\mathbb{T}^{3}$ compact we do not require a passage to subsequences on the parameter $\alpha$ but the convergence holds true for the entire sequence. Next we can say that

$$
\begin{aligned}
\mathcal{Q}^{\varepsilon}\left(U_{\alpha}^{\varepsilon}, U_{\alpha}^{\varepsilon}\right)-\mathcal{Q}\left(U_{\alpha}, U_{\alpha}\right)= & \left(\mathcal{Q}^{\varepsilon}\left(U_{\alpha}^{\varepsilon}, U_{\alpha}^{\varepsilon}\right)-\mathcal{Q}^{\varepsilon}\left(U_{\alpha}, U_{\alpha}\right)\right) \\
& +\left(\mathcal{Q}^{\varepsilon}\left(U_{\alpha}, U_{\alpha}\right)-\mathcal{Q}\left(U_{\alpha}, U_{\alpha}\right)\right)
\end{aligned}
$$

and again, for $\alpha>0$ fixed

$$
\mathcal{Q}^{\varepsilon}\left(U_{\alpha}^{\varepsilon}, U_{\alpha}^{\varepsilon}\right)-\mathcal{Q}^{\varepsilon}\left(U_{\alpha}, U_{\alpha}\right) \stackrel{\varepsilon \rightarrow 0}{\longrightarrow} 0,
$$


weakly since $U_{\alpha}^{\varepsilon} \stackrel{\varepsilon \rightarrow 0}{\longrightarrow} U_{\alpha}$ in $L_{\mathrm{loc}}^{2}\left(\mathbb{R}_{+} ; H^{-\eta}\right)$ due to the topological argument performed in Proposition 5.1. Finally we can apply the nonstationary phase theorem on $\mathcal{Q}^{\varepsilon}\left(U_{\alpha}, U_{\alpha}\right)-\mathcal{Q}\left(U_{\alpha}, U_{\alpha}\right)$ deducing that

$$
\mathcal{Q}^{\varepsilon}\left(U_{\alpha}, U_{\alpha}\right)-\mathcal{Q}\left(U_{\alpha}, U_{\alpha}\right) \stackrel{\varepsilon \rightarrow 0}{\longrightarrow} 0,
$$

in the sense of distributions for $\alpha>0$ fixed. Whence [5.3)-[5.6 imply that, fixed a (possibly small) positive $\alpha>0$, considering a $\phi \in \mathcal{D}\left(\mathbb{R}_{+} \times \mathbb{T}^{3}\right)$, there exists a $c_{\alpha}=c_{\alpha}(\phi)>0$ such that $c_{\alpha} \rightarrow 0$ as $\alpha \rightarrow 0$ and such that

$$
\lim _{\varepsilon \rightarrow 0}\left|\int_{\mathbb{R}_{+} \times \mathbb{T}^{3}}\left(\mathcal{Q}^{\varepsilon}\left(U^{\varepsilon}, U^{\varepsilon}\right)-\mathcal{Q}(U, U)\right) \cdot \phi \mathrm{d} x \mathrm{~d} t\right| \leqslant c_{\alpha} .
$$

The left-hand side of [5.7] is indeed independent from the parameter $\alpha$, whence

$$
\lim _{\varepsilon \rightarrow 0}\left|\int_{\mathbb{R}_{+} \times \mathbb{T}^{3}}\left(\mathcal{Q}^{\varepsilon}\left(U^{\varepsilon}, U^{\varepsilon}\right)-\mathcal{Q}(U, U)\right) \cdot \phi \mathrm{d} x \mathrm{~d} t\right| \leqslant \lim _{\alpha \rightarrow 0} c_{\alpha}=0
$$

We underline the fact that the following calculations are an adaptation of the ones present in the work [24] to the case of anisotropic viscosity. For this reason many calculations shall not be carried out in detail, or we shall directly refer to the work $[24]$ and references therein.

Once the convergence for the bilinear interactions is formalized we focus to understand how the global splitting introduced in Section 3.1 can be applied on bilinear interactions of elements which are not smooth.

P. Embid and A. Majda proved the following lemma in [22]:

Lemma 5.3. $\mathcal{F}^{-1}\left(\left(\left.\mathcal{F} \mathcal{Q}^{\varepsilon}\left(U^{\varepsilon}, U^{\varepsilon}\right)|| n\right|_{F} e^{0}(n)\right)_{\mathbb{C}^{4}}\right) \stackrel{\varepsilon \rightarrow 0}{\longrightarrow} v_{\mathrm{QG}} \cdot \nabla \Omega$. The limit holds in the sense of distributions.

Proof. Let us compute

$$
\begin{aligned}
\mathcal{F}^{-1}\left(\left(\left.\mathcal{F} \mathcal{Q}^{\varepsilon}\left(U^{\varepsilon}, U^{\varepsilon}\right)|| n\right|_{F} e^{0}(n)\right)_{\mathbb{C}^{4}}\right)-v_{\mathrm{QG}} & \cdot \nabla \Omega \\
= & \mathcal{F}^{-1}\left(\left(\left.\mathcal{F} \mathcal{Q}^{\varepsilon}\left(U^{\varepsilon}, U^{\varepsilon}\right)|| n\right|_{F} e^{0}(n)\right)_{\mathbb{C}^{4}}\right)-\mathcal{F}^{-1}\left(\left(\left.\mathcal{F} \mathcal{Q}^{\varepsilon}\left(U_{\alpha}^{\varepsilon}, U_{\alpha}^{\varepsilon}\right)|| n\right|_{F} e^{0}(n)\right)_{\mathbb{C}^{4}}\right) \\
& +\mathcal{F}^{-1}\left(\left(\left.\mathcal{F} \mathcal{Q}^{\varepsilon}\left(U_{\alpha}^{\varepsilon}, U_{\alpha}^{\varepsilon}\right)|| n\right|_{F} e^{0}(n)\right)_{\mathbb{C}^{4}}\right)-v_{\mathrm{QG}, \alpha} \cdot \nabla \Omega_{\alpha} \\
& +v_{\mathrm{QG}, \alpha} \cdot \nabla \Omega_{\alpha}-v_{\mathrm{QG}} \cdot \nabla \Omega .
\end{aligned}
$$

The element

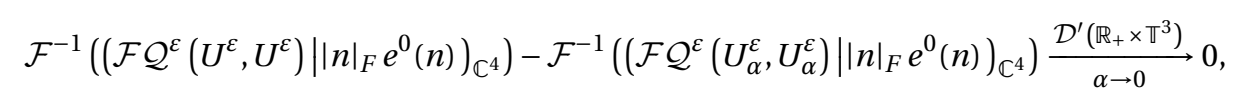

since $U_{\alpha}^{\varepsilon} \stackrel{\alpha \rightarrow 0}{\longrightarrow} U^{\varepsilon}$ in $L_{\mathrm{loc}}^{\infty}\left(\mathbb{R}_{+} ; L^{2}\right)$. Next applying the nonstationary phase theorem and Lemma 3.1 we can say that

$$
\mathcal{F}^{-1}\left(\left(\left.\mathcal{F} \mathcal{Q}^{\varepsilon}\left(U_{\alpha}^{\varepsilon}, U_{\alpha}^{\varepsilon}\right)|| n\right|_{F} e^{0}(n)\right)_{\mathbb{C}^{4}}\right)-v_{\mathrm{QG}, \alpha} \cdot \nabla \Omega_{\alpha} \rightarrow 0,
$$

as $\varepsilon \rightarrow 0$ in the sense of distributions. Lastly again we can argue as above in order to state that

$$
v_{\mathrm{QG}, \alpha} \cdot \nabla \Omega_{\alpha}-v_{\mathrm{QG}} \cdot \nabla \Omega \underset{\mathcal{D}^{\prime}\left(\mathbb{R}_{+} \times \mathbb{T}^{3}\right)}{\stackrel{\varepsilon \rightarrow 0}{\longrightarrow}} 0,
$$

since $v_{\mathrm{QG}, \alpha} \rightarrow v_{\mathrm{QG}}$ and $\Omega_{\alpha} \rightarrow \Omega$ in $L_{\mathrm{loc}}^{\infty}\left(\mathbb{R}_{+} ; L^{2}\right)$, concluding.

We want to understand which are the projections of $\mathbb{D}^{\varepsilon} U$ onto the oscillatory and non oscillatory space as $\varepsilon \rightarrow 0$. This is easily done if we consider the formulation of the limit form as it is given in (3.15). Let us consider the projection of the limit linear form onto the potential space defined by $\Omega=\mathcal{F}^{-1}\left(\left(\left.\mathcal{F} U|| n\right|_{F} e^{0}\right)_{\mathbb{C}^{4}}\right)$,

$$
\left(\left.\mathcal{F} \mathbb{D} U|| n\right|_{F} e^{0}\right)_{\mathbb{C}^{4}}=\sum_{\omega_{n}^{a, b}=0}\left(\mathbf{D}(n) U^{b}(n) \mid e^{a}(n)\right)_{\mathbb{C}^{4}}\left(\left.e^{a}(n)|| n\right|_{F} e^{0}\right)_{\mathbb{C}^{4}}
$$

As it has been pointed out above $e^{0} \perp e^{ \pm}$, hence $a=0$. On the other hand if we consider the limit set $\omega_{n}^{a, b}=0$ with the fact that $a=0$ we easily obtain that $\omega^{b}(n) \equiv 0$, whence $b=0$ as well, hence we obtained that

$$
\left(-\mathcal{L}\left(-\frac{t}{\varepsilon}\right) \mathbf{D} \mathcal{L}\left(\frac{t}{\varepsilon}\right) U^{\varepsilon} \mid \mathcal{F}^{-1}\left(\left|n_{F}\right| e^{0}\right)\right)_{\mathbb{C}^{4}} \stackrel{\varepsilon \rightarrow 0}{\longrightarrow} a_{\mathrm{QG}}\left(D_{h}\right) \Omega=\quad \mathcal{F}^{-1}\left(\frac{v\left(n_{1}^{2}+n_{2}^{2}\right)+v^{\prime} F^{2} n_{3}^{2}}{n_{1}^{2}+n_{2}^{2}+F^{2} n_{3}^{2}}\left(n_{1}^{2}+n_{2}^{2}\right) \widehat{\Omega}_{n}\right) .
$$


In the same way, defining $U^{a}=\left(\mathcal{F} U \mid e^{a}\right) e^{a}$

$$
-\lim _{\varepsilon \rightarrow 0} \mathcal{L}\left(-\frac{t}{\varepsilon}\right) \mathbf{D} \mathcal{L}\left(\frac{t}{\varepsilon}\right) U_{\mathrm{osc}}^{\varepsilon}=a_{\mathrm{osc}}\left(D_{h}\right) U_{\mathrm{osc}}^{\varepsilon}=\mathcal{F}^{-1}\left(\sum_{\substack{\omega_{n}^{a, b}=0 \\ a, b= \pm}}\left(\mathbf{D}(n) U^{b}(n) \mid e^{a}(n)\right)_{\mathbb{C}^{4}} e^{a}(n)\right) .
$$

We want now to understand which form assumes the limit as $\varepsilon \rightarrow 0$ of the projection of $\mathcal{Q}^{\varepsilon}\left(U^{\varepsilon}, U^{\varepsilon}\right)$ onto the oscillatory subspace $\mathbb{C} e^{-} \oplus \mathbb{C} e^{+}$. In particular the following result holds true:

Lemma 5.4. For every three-dimensional torus $\mathbb{T}^{3}$ we have

$$
\mathcal{Q}^{\varepsilon}\left(U^{\varepsilon}, U^{\varepsilon}\right)_{\mathrm{osc}} \stackrel{\varepsilon \rightarrow 0}{\longrightarrow}\left(\mathcal{Q}\left(V_{\mathrm{QG}}, U_{\mathrm{osc}}\right)\right)_{\mathrm{osc}}+\left(\mathcal{Q}\left(U_{\mathrm{osc}}, V_{\mathrm{QG}}\right)\right)_{\mathrm{osc}}+\left(\mathcal{Q}\left(U_{\mathrm{osc}}, U_{\mathrm{osc}}\right)\right)_{\mathrm{osc}}
$$

Proof. We avoid to give a detailed proof of such result since the proof is very similar to the one performed in Lemma 5.3 but using Corollary 3.4 instead of Lemma 3.1

The above lemmas hence states that, in the limit $\varepsilon \rightarrow 0$, there is no bilinear interaction of kernel elements in the equation describing the evolution of $U_{\text {osc. }}$.

The filtered system $\overline{\mathrm{FS}_{\varepsilon}}$ can be described, as $\varepsilon \rightarrow 0$, thanks to the following two systems:

$$
\begin{aligned}
& \left\{\begin{array}{l}
\partial_{t} \Omega+v_{\mathrm{QG}}^{h} \cdot \nabla_{h} \Omega+a_{\mathrm{QG}}\left(D_{h}\right) \Omega=0 \\
\operatorname{div}_{h} v_{\mathrm{QG}}^{h}=\operatorname{div} v_{\mathrm{QG}}=0 \\
\left.\Omega\right|_{t=0}=\Omega_{0}
\end{array}\right. \\
& \left\{\begin{array}{rl}
\partial_{t} U_{\mathrm{osc}}+ & \left(\mathcal{Q}\left(V_{\mathrm{QG}}, U_{\mathrm{osc}}\right)\right)_{\mathrm{osc}}+\left(\mathcal{Q}\left(U_{\mathrm{osc}}, V_{\mathrm{QG}}\right)\right)_{\mathrm{osc}} \\
\quad & \left(\mathcal{Q}\left(U_{\mathrm{osc}}, U_{\mathrm{osc}}\right)\right)_{\mathrm{osc}}+a_{\mathrm{osc}}\left(D_{h}\right) U_{\mathrm{osc}}=0 \\
\operatorname{div} u_{\mathrm{osc}}=0 & 0 \\
\left.U_{\mathrm{osc}}\right|_{t=0}=U_{\mathrm{osc}, 0}=\left(V_{0}\right)_{\mathrm{osc}} .
\end{array}\right.
\end{aligned}
$$

The system (5.9) represents the projection of the limit system onto the non-oscillatory potential subspace defined by $\Omega$, and (5.10) represents the projection onto $\mathbb{C} e^{-} \oplus \mathbb{C} e^{+}$.

It is easy to deduce from [5.9] that if $V_{\mathrm{QG}}=\left(\begin{array}{c}\nabla_{h}^{\perp} \\ 0 \\ -F \partial_{3}\end{array}\right) \Delta_{F}^{-1} \Omega$ then

$$
\left\{\begin{array}{l}
\partial_{t} V_{\mathrm{QG}}+a_{\mathrm{QG}}\left(D_{h}\right) V_{\mathrm{QG}}=-\left(\begin{array}{c}
\nabla_{h}^{\perp} \\
0 \\
-\partial_{3} F
\end{array}\right) \Delta_{F}^{-1}\left(v_{\mathrm{QG}}^{h} \cdot \nabla_{h} \Omega\right), \\
\operatorname{div}_{h} v_{\mathrm{QG}}^{h}=\operatorname{div} v_{\mathrm{QG}}=0 \\
\left.V_{\mathrm{QG}}\right|_{t=0}=V_{\mathrm{QG}, 0}=\left(\nabla_{h}^{\perp}, 0,-F \partial_{3}\right)^{\top} \Delta_{F}^{-1} \Omega_{0} .
\end{array}\right.
$$

We remark that in the equation $[5.10)$ the term $\mathcal{Q}\left(U_{\mathrm{osc}}, U_{\mathrm{osc}}\right)$ represents a bilinear interaction between highly oscillating modes, i.e. we are taking into account some potentially resonant effect such as in [35].

The following lemma gives a connection in terms of regularity between the solutions of [5.9] and [5.11], and will result to be extremely useful in the energy estimates for the global well posedness of the limit system.

Lemma 5.5. Let $\Lambda_{h}^{s} \Lambda_{v}^{s^{\prime}} \Omega \in L^{2}\left(\mathbb{T}^{3}\right)$, with $V_{\mathrm{QG}}=\left(\begin{array}{c}\nabla_{h}^{\perp} \\ 0 \\ -F \partial_{3}\end{array}\right) \Delta_{F}^{-1} \Omega$. Let $\sigma \in[0,1]$, then there exists a uniformly finite (in $\sigma$ ) constant $C_{\sigma}$ depending only on $\sigma$ such that

$$
\left\|\Lambda_{h}^{s+\sigma} \Lambda_{v}^{s^{\prime}+(1-\sigma)} v_{\mathrm{QG}}\right\|_{L^{2}\left(\mathbb{T}^{3}\right)} \leqslant C_{\sigma}\left\|\Lambda_{h}^{s} \Lambda_{v}^{s^{\prime}} \Omega\right\|_{L^{2}\left(\mathbb{T}^{3}\right)}
$$

\subsection{Propagation of the horizontal average}

In the following lemmas we identify some conditions which suffice to guarantee that the horizontal average of $U=$ $U_{\mathrm{QG}}+U_{\mathrm{osc}}$ solution of the limit system $[5.10$ - 5.11 is preserved for each time $t>0$. It is important to propagate the horizontal average since, generally, we cannot use inequalities such as the one stated in 2.7) or Corollary 2.5 unless the horizontal mean of the function considered is nil. It is in this setting that the condition $[(\mathcal{P})$ plays a fundamental role. 
Lemma 5.6. Let $V_{\mathrm{QG}}$ the solution of [5.11, if we define

$$
\underline{V_{\mathrm{QG}}}\left(t, x_{3}\right)=\frac{1}{\left|\mathbb{T}_{h}^{2}\right|} \int_{\mathbb{T}_{h}^{2}} V_{\mathrm{QG}}\left(t, y_{h}, x_{3}\right) \mathrm{d} y_{h},
$$

then

$$
\partial_{t} \underline{V_{\mathrm{QG}}}\left(t, x_{3}\right)=0
$$

Proof. It suffice to remark that

$$
\left(-\partial_{2}, \partial_{1}, 0,-F \partial_{3}\right)^{\top} \Delta_{F}^{-1}\left(v_{\mathrm{QG}}^{h} \cdot \nabla_{h} \Omega\right)=\left(-\partial_{2}, \partial_{1}, 0,-F \partial_{3}\right)^{\top} \Delta_{F}^{-1} \operatorname{div}_{h}\left(v_{\mathrm{QG}}^{h} \Omega\right) .
$$

Lemma 5.7. Let $U$ be the weak solution of the limit system $[\mathrm{S})$, the if $\mathbb{T}^{3}$ satisfies the condition $(\mathcal{P})$

$$
\partial_{t} \int_{\mathbb{T}_{h}^{2}} U\left(t, x_{h}, x_{3}\right) \mathrm{d} x_{h}=0
$$

Proof. Taking in consideration the oscillatory part described by equation [5.10] it suffices to prove that

$$
\int_{\mathbb{T}_{h}^{2}} \mathcal{Q}\left(V_{\mathrm{QG}}, U_{\mathrm{osc}}\right) \mathrm{d} x_{h}=\int_{\mathbb{T}_{h}^{2}} \mathcal{Q}\left(U_{\mathrm{osc}}, V_{\mathrm{QG}}\right) \mathrm{d} x_{h}=\int_{\mathbb{T}_{h}^{2}} \mathcal{Q}\left(U_{\mathrm{osc}}, U_{\mathrm{osc}}\right) \mathrm{d} x_{h}=0,
$$

we consider at first the term $\int_{\mathbb{T}_{h}^{2}} \mathcal{Q}\left(V_{\mathrm{QG}}, U_{\mathrm{Osc}}\right) \mathrm{d} x_{h}$. To do so we consider

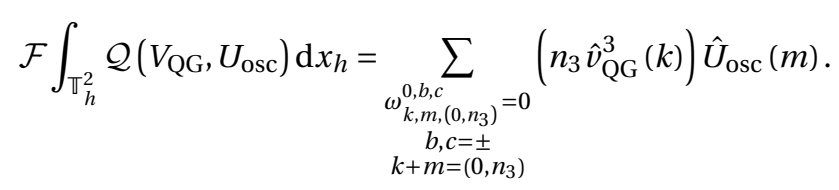

If we look what the term $\hat{v}_{\mathrm{QG}}^{3}(k)$ is we can easily deduce that $\hat{v}_{\mathrm{QG}}^{3}(k)=\hat{V}_{\mathrm{QG}}(k) \cdot e^{0}(k) e^{0,3}(k)$, where $e^{0}$ is defined in (3.4) and $e^{0,3}$ is the third component of $e^{0}$. Looking at 3.4] we immediately notice that $e^{0,3} \equiv 0$, and hence the above value is null.

Next we consider the following term

$$
\mathcal{F} \int_{\mathbb{T}_{h}^{2}} \mathcal{Q}\left(U_{\mathrm{Osc}}, V_{\mathrm{QG}}\right) \mathrm{d} x_{h}=\sum_{\substack{\omega^{a, 0, c}, \ldots, m,\left(0, n_{3}\right) \\ a, c= \pm k+m=\left(0, n_{3}\right)}}\left(\left(\left(n_{3} \hat{u}_{\mathrm{osc}}^{3}(k)\right) \hat{V}_{\mathrm{QG}}(m)\right) \mid e^{c}(n)\right)_{\mathbb{C}^{4}} e^{c}(n) .
$$

In order to prove that the above quantity is zero we have to study the summation set. Recall that the eigenvalues are given in 3.4. The right hand side of [5.12 was evaluated explicitly thanks to the explicit formulation of the bilinear form $\mathcal{Q}$. The formulation of the summation set is quite simple thanks to the relation $n_{h} \equiv 0$, writing down in fact explicitly the relation $\omega_{k,\left(0, n_{3}\right)-k,\left(0, n_{3}\right)}^{a, 0, c}=01$ we deduce that we are considering the following modes:

$$
\mathcal{K}_{ \pm}=\left\{k \in \mathbb{Z}^{3} \mid \omega^{ \pm}(k)=1\right\} .
$$

The equation $\omega^{ \pm}(k)=1$ characterizing $\mathcal{K}_{ \pm}$reads as

$$
\frac{\left(F^{2} \check{k}_{3}^{2}+\left|\check{k}_{h}\right|^{2}\right)^{1 / 2}}{|\check{k}|}= \pm F
$$

which is equivalent to

$$
\left(F^{2}-1\right)\left|k_{h}\right|^{2}=0
$$

It is easy to deduce that this relation is satisfied only if $k_{h} \equiv 0$, but let us consider now in detail what the element $\left.\hat{u}_{\mathrm{osc}}^{3}(k)\right|_{k=\left(0, k_{3}\right)}$ appearing in (5.12) is. By definition $\hat{u}_{\mathrm{osc}}^{3}(k)=\left(\mathcal{F} U(k) \mid e^{ \pm}(k)\right) e^{ \pm, 3}(k)$, where $e^{ \pm, 3}(k)$ is the third component of the oscillating eigenvectors defined in [3.6), i.e. $e^{ \pm, 3}(k) \equiv 0$. Whence $\hat{u}_{\mathrm{osc}}^{3}\left(0, k_{3}\right) \equiv 0$ and this implies that the contribution in [5.12] is zero.

\footnotetext{
${ }^{1}\left(0, n_{3}\right)-k=m$ and we recover the same summation set as in 5.12 .
} 
Next we deal with the more complex term, namely the term

$$
\int_{\mathbb{T}_{h}^{2}}\left(\mathcal{Q}\left(U_{\mathrm{osc}}, U_{\mathrm{osc}}\right)\right)_{\mathrm{osc}} \mathrm{d} x_{h}
$$

For this term the resonance set defined in Definition 1.6 plays a fundamental role, let us consider the explicit expression of the above term

$$
\int_{\mathbb{T}_{h}^{2}}\left(\mathcal{Q}\left(U_{\mathrm{osc}}, U_{\mathrm{osc}}\right)\right)_{\mathrm{osc}} \mathrm{d} x_{h}=\mathcal{F}^{-1}\left(\sum_{\mathcal{K}_{\left(0, \check{n}_{3}\right)}^{\star}}\left(\sum_{j=1,2,3} U^{a, j}(k) m_{j} U^{b}(m) \mid e^{c}\left(0, n_{3}\right)\right)_{\mathbb{C}^{4}} e^{c}\left(0, n_{3}\right)\right) .
$$

We prove that the above quantity is zero by proving that $\mathcal{K}_{\left(0, \check{n}_{3}\right)}^{\star}=\varnothing$. Since $\check{n}_{h}=0$ and the convolution constraint $\check{k}+\check{m}=\check{n}$ we deduce that $\check{k}_{h}+\check{m}_{h}=0$, i.e. $\left|\check{k}_{h}\right|=\left|\check{m}_{h}\right|=\lambda$. Writing down the resonant equation we obtain the following equality

$$
\frac{\left(F^{2} \check{k}_{3}^{2}+\lambda^{2}\right)^{1 / 2}}{\left(\lambda^{2}+\check{k}_{3}^{2}\right)^{1 / 2}} \pm \frac{\left(F^{2} \check{m}_{3}^{2}+\lambda^{2}\right)^{1 / 2}}{\left(\lambda^{2}+\check{m}_{3}^{2}\right)^{1 / 2}}= \pm 1
$$

Taking square (twice) and after some algebraic manipulation we obtain that the above equation is equivalent to

$$
\left(\lambda^{4}+F^{2} \lambda^{2} \check{m}_{3}^{2}+\check{k}_{3}^{2}\left(-\left(-2+F^{2}\right) \lambda^{2}+\check{m}_{3}^{2}\right)\right)^{2}=4\left(\lambda^{2}+\check{k}_{3}^{2}\right)^{2}\left(\lambda^{2}+\check{m}_{3}^{2}\right)\left(\lambda^{2}+F^{2} \check{m}_{3}^{2}\right)
$$

We multiply the above equation for $a_{3}^{8}$, obtaining the new equality in the unknown $\mu^{2}=\lambda^{2} a_{3}^{2}$

$$
\left(\mu^{4}+F^{2} \mu^{2} m_{3}^{2}+k_{3}^{2}\left(-\left(-2+F^{2}\right) \mu^{2}+m_{3}^{2}\right)\right)^{2}=4\left(\mu^{2}+k_{3}^{2}\right)^{2}\left(\mu^{2}+m_{3}^{2}\right)\left(\mu^{2}+F^{2} m_{3}^{2}\right),
$$

and

$$
\mu^{2}=\lambda^{2} a_{3}^{2}=\left(\frac{a_{3}}{a_{1}}\right)^{2} k_{1}^{2}+\left(\frac{a_{3}}{a_{2}}\right)^{2} k_{2}^{2}=\mu_{1} k_{1}^{2}+\mu_{2} k_{2}^{2} .
$$

Since the torus satisfies the Condition $(\mathcal{P})$ we know that $F^{2}=r_{1} / r_{2} \in \mathbb{Q}$, hence we can transform the expression in (5.13) in an equation of the form $P(\mu)=0$, with $P \in \mathbb{Z}[\mu]$. Whence by the definition of Condition $(\mathcal{P})$ given in Definition 1.8 we argue that

- If $\mu_{1}=a_{3}^{2} / a_{1}^{2} \in \mathbb{Q}$ the [5.13 can be rewritten as $\tilde{P}\left(\mu_{2}\right)=0$ where $\operatorname{deg} \tilde{P}=4$. By hypothesis in Definition $1.8 \mu_{2}$ is not algebraic of degree smaller or equal than four, this implies that the equation $\tilde{P}\left(\mu_{2}\right)=0$ has no solution, concluding.

- If $\mu_{2}=a_{3}^{2} / a_{2}^{2} \in \mathbb{Q}$ the procedure is the same as above, but symmetric (see Definition 1.8 .

We have hence identified some conditions under such we can say that the horizontal mean of the limit function $U=\lim _{\varepsilon \rightarrow 0} \mathcal{L}\left(-\frac{t}{\varepsilon}\right) V^{\varepsilon}$ is preserved. Hence if we consider initial data with zero horizontal average we can use freely 2.7) and the following Poincaré inequality $\|U\|_{L^{p}\left(\mathbb{T}^{3}\right)} \leqslant C\left\|\nabla_{h} U\right\|_{L^{p}\left(\mathbb{T}^{3}\right)}$, holds.

\section{Propagation of $H^{0, s}$ regularity}

\subsection{The quasi-geostrophic part}

Subsection 4 ensures us that there exists a solution $U$ for the limit system $[$ which is

$$
U \in L^{\infty}\left(\mathbb{R}_{+} ; L^{2}\left(\mathbb{T}^{3}\right)\right) \quad \nabla_{h} U \in L^{2}\left(\mathbb{R}_{+} ; L^{2}\left(\mathbb{T}^{3}\right)\right) .
$$

The scope of the present and following section is to prove if, under suitable initial conditions, the equations (5.9) and 5.10 propagate $H^{0, s}, s \geqslant 1$ regularity.

Proposition 6.1. Let $\Omega_{0} \in L^{2}\left(\mathbb{T}^{3}\right)$ then $\Omega \in L^{\infty}\left(\mathbb{R}_{+} ; L^{2}\left(\mathbb{T}^{3}\right)\right), \nabla_{h} \Omega \in L^{2}\left(\mathbb{R}_{+} ; L^{2}\left(\mathbb{T}^{3}\right)\right)$, and in particular for each $t>0$ the following bound holds true

$$
\|\Omega(t)\|_{L^{2}\left(\mathbb{T}^{3}\right)}^{2}+2 c \int_{0}^{t}\left\|\nabla_{h} \Omega(\tau)\right\|_{L^{2}\left(\mathbb{T}^{3}\right)}^{2} \mathrm{~d} \tau \leqslant C\left\|\Omega_{0}\right\|_{L^{2}\left(\mathbb{T}^{3}\right)}^{2} .
$$
1.10 .

This is a standard $L^{2}$ energy estimate on the parabolic equation [5.9] which has been already proved in Theorem 
Proposition 6.2. Let $\Omega$ be the solution of 5.9 and let $\Omega_{0} \in H^{0, s}$ for some $s>0$. Then for all $t \in \mathbb{R}, \Omega \in \mathcal{C}\left(\mathbb{R}_{+} ; H^{0, s}\right)$ and $\nabla_{h} \Omega \in L^{2}\left(\mathbb{R}_{+} ; H^{0, s}\right)$. The following estimate holds:

$$
\|\Omega(t)\|_{H^{0, s}}^{2}+c \int_{0}^{t}\left\|\nabla_{h} \Omega(\tau)\right\|_{H^{0, s}}^{2} \mathrm{~d} \tau \leqslant C\left\|\Omega_{0}\right\|_{H^{0, s}} \exp \left\{\frac{2 C}{c}\left(1+\left\|\Omega_{0}\right\|_{L^{2}\left(\mathbb{T}^{3}\right)}^{2}\right)\left\|\Omega_{0}\right\|_{L^{2}\left(\mathbb{T}^{3}\right)}^{2}\right\}
$$

Proof. Applying the vertical truncation $\triangle_{q}^{\mathrm{v}}$ on both sides of equation [5.9], multiplying both sides for $\triangle_{q}^{\mathrm{v}} \Omega$ and taking the scalar product in $L^{2}\left(\mathbb{T}^{3}\right)$ we obtain

$$
\frac{1}{2} \frac{\mathrm{d}}{\mathrm{d} t}\left\|\triangle_{q}^{\mathrm{v}} \Omega\right\|_{L^{2}\left(\mathbb{T}^{3}\right)}^{2}+c\left\|\triangle_{q}^{\mathrm{v}} \nabla_{h} \Omega\right\|_{L^{2}\left(\mathbb{T}^{3}\right)}^{2} \leqslant\left|\left(\triangle_{q}^{\mathrm{v}}\left(v_{\mathrm{QG}}^{h} \cdot \nabla_{h} \Omega\right) \mid \triangle_{q}^{\mathrm{v}} \Omega\right)_{L^{2}\left(\mathbb{T}^{3}\right)}\right| .
$$

By use of Cauchy-Schwartz inequality and 8.1 we obtain

$$
\begin{aligned}
& \frac{1}{2} \frac{\mathrm{d}}{\mathrm{d} t}\left\|\triangle_{q}^{\mathrm{v}} \Omega\right\|_{L^{2}\left(\mathbb{T}^{3}\right)}^{2} \\
& \quad \leq c\left\|\triangle_{q}^{\mathrm{v}} \nabla_{h} \Omega\right\|_{L^{2}\left(\mathbb{T}^{3}\right)}^{2} \\
& \quad \leq C 2^{-2 q s} b_{q}(t)\left[\|\Omega\|_{L^{2}\left(\mathbb{T}^{3}\right)}^{1 / 2}\left\|\nabla_{h} \Omega\right\|_{L^{2}\left(\mathbb{T}^{3}\right)}^{1 / 2}\|\Omega\|_{H^{0, s}}^{1 / 2}\left\|\nabla_{h} \Omega\right\|_{H^{0, s}}^{3 / 2}+\left\|\nabla_{h} \Omega\right\|_{L^{2}\left(\mathbb{T}^{3}\right)}\|\Omega\|_{H^{0, s}}\left\|\nabla_{h} \Omega\right\|_{H^{0, s}}\right]
\end{aligned}
$$

We recall that in $6.2\left(b_{q}\right)_{q}$ is a $\ell^{1}(\mathbb{Z})$ positive sequence which depends on $\Omega$ and such that $\sum_{q} b_{q}(t) \leqslant 1$. Multiplying equation 6.2 on both sides for $2^{2 q s}$, summing on $q \in \mathbb{Z}$ and using the convexity inequalities $2 a b \leqslant a^{2}+b^{2}$ and $a b \leqslant$ $\frac{1}{4} a^{4}+\frac{3}{4} b^{4 / 3}$ we obtain

$$
\frac{1}{2} \frac{\mathrm{d}}{\mathrm{d} t}\|\Omega\|_{H^{0, s}}^{2}+c\left\|\nabla_{h} \Omega\right\|_{H^{0, s}}^{2} \leqslant \frac{c}{2}\left\|\nabla_{h} \Omega\right\|_{H^{0, s}}^{2}+C\left(\left(1+\|\Omega\|_{L^{2}\left(\mathbb{T}^{3}\right)}^{2}\right)\left\|\nabla_{h} \Omega\right\|_{L^{2}\left(\mathbb{T}^{3}\right)}^{2}\right)\|\Omega\|_{H^{0, s}}^{2}
$$

whence, applying Gronwall inequality to 6.3 in $[0, t]$ we deduce the bound

$$
\|\Omega(t)\|_{H^{0, s}}^{2}+c \int_{0}^{t}\left\|\nabla_{h} \Omega(\tau)\right\|_{H^{0, s}}^{2} \mathrm{~d} \tau \leqslant C\left\|\Omega_{0}\right\|_{H^{0, s}} \exp \left\{2 C \int_{0}^{t}\left(1+\|\Omega(s)\|_{L^{2}\left(\mathbb{T}^{3}\right)}^{2}\right)\left\|\nabla_{h} \Omega(s)\right\|_{L^{2}\left(\mathbb{T}^{3}\right)}^{2} \mathrm{~d} s\right\} .
$$

Hence, considering that $\Omega$ is bounded in $L^{\infty}\left(\mathbb{R}_{+} ; L^{2}\left(\mathbb{T}^{3}\right)\right)$ and $\nabla_{h} \Omega$ is bounded in $L^{2}\left(\mathbb{R}_{+} ; L^{2}\left(\mathbb{T}^{3}\right)\right)$ we deduce the estimate 6.1.

Remark 6.3. In Proposition 6.2 we do not require the initial data to be of zero horizontal average in order to propagate $H^{0, s}$ norms.

\subsection{The oscillatory part}

We can now turn our attention on the oscillatory part $U_{\text {osc }}$ solution of the equation [5.10]. Indeed the terms $\mathcal{Q}\left(V_{\mathrm{QG}}, U_{\mathrm{osc}}\right)$ and $\mathcal{Q}\left(U_{\mathrm{osc}}, V_{\mathrm{QG}}\right)$ present in 5.10 ) should not present a problem in the propagation of regularity, being linear in $U_{\mathrm{osc}}$. The term $\mathcal{Q}\left(U_{\mathrm{osc}}, U_{\mathrm{osc}}\right)$ though is a bilinear interaction of oscillating modes. Fortunately as pointed out in Lemma 8.4 the bilinear form $\mathcal{Q}$ has better product rules than the standard Navier-Stokes bilinear form, this will allow us to recover the global well posedness result for 5.10 .

Lemma 6.4. Let $U$ be the weak solution defined in Theorem 1.10 then $U_{\mathrm{osc}}=U-V_{\mathrm{QG}}$ satisfies the energy bound

$$
\left\|U_{\mathrm{osc}}(t)\right\|_{L^{2}\left(\mathbb{J}^{3}\right)}^{2}+c \int_{0}^{t}\left\|\nabla_{h} U_{\mathrm{osc}}(\tau)\right\|_{L^{2}\left(\mathbb{T}^{3}\right)}^{2} \mathrm{~d} \tau \leqslant C\left\|U_{0}\right\|_{L^{2}\left(\mathbb{J}^{3}\right)}^{2} .
$$

Proof. The proof stems from the fact that $U_{\mathrm{osc}}=\Pi_{\mathrm{osc}} U$ where $\Pi_{\mathrm{osc}}=1-\Pi_{\mathrm{QG}}$ is a pseudo-differential operator of order zero as it was explained in the proof of Theorem 1.10 .

Proposition 6.5. Let $U_{\mathrm{osc}}$ be the solution of $\left[5.10\right.$ ) and $V_{\mathrm{QG}, 0}, U_{\mathrm{osc}, 0}=0$. Let $\mathbb{T}^{3}$ satisfy the condition $(\mathcal{P})$ and $U_{\mathrm{osc}, 0}, \Omega_{0} \in$ $H^{0, s}$ for $s>1 / 2$, then $U_{\mathrm{osc}} \in \mathcal{C}\left(\mathbb{R}_{+} ; H^{0, s}\right)$ and $\nabla_{h} U_{\mathrm{osc}} \in \overline{L^{2}\left(\mathbb{R}_{+}\right.} ; \overline{\left.H^{0, s}\right)}$ and the following bound holds

$$
\begin{aligned}
& \left\|U_{\mathrm{osc}}(t)\right\|_{H^{0, s}}^{2}+c \int_{0}^{t}\left\|U_{\mathrm{osc}}(\tau)\right\|_{H^{0, s}}^{2} \mathrm{~d} \tau \\
& \quad \leqslant C\left\|U_{\mathrm{osc}, 0}\right\|_{H^{0, s}}^{2} \exp \left\{\frac{2 C}{c}\left[\left\|\Omega_{0}\right\|_{H^{0, s}} \exp \left\{\frac{2 C}{c}\left(1+\left\|\Omega_{0}\right\|_{L^{2}\left(\mathbb{T}^{3}\right)}^{2}\right)\left\|\Omega_{0}\right\|_{L^{2}\left(\mathbb{T}^{3}\right)}^{2}\right\}+\left(1+\left\|U_{0}\right\|_{L^{2}\left(\mathbb{T}^{3}\right)}^{2}\right)\left\|U_{0}\right\|_{L^{2}\left(\mathbb{T}^{3}\right)}^{2}\right]\right\} .
\end{aligned}
$$

Proof. As in the proof of Proposition 6.2 apply the vertical truncation $\triangle_{q}^{\mathrm{v}}$ on both sides of 5.10 and taking scalar product in $L^{2}\left(\mathbb{T}^{3}\right)$ we obtain

$$
\begin{aligned}
\frac{1}{2} \frac{\mathrm{d}}{\mathrm{d} t}\left\|\triangle_{q}^{\mathrm{v}} U_{\mathrm{osc}}\right\|_{L^{2}\left(\mathbb{T}^{3}\right)}^{2}+c\left\|\triangle_{q}^{\mathrm{v}} \nabla_{h} U_{\mathrm{osc}}\right\|_{L^{2}\left(\mathbb{T}^{3}\right)}^{2} \leqslant\left|\left(\triangle_{q}^{\mathrm{v}} \mathcal{Q}\left(V_{\mathrm{QG}}, U_{\mathrm{osc}}\right) \mid \triangle_{q}^{\mathrm{v}} U_{\mathrm{osc}}\right)\right| \\
+\left|\left(\triangle_{q}^{\mathrm{v}} \mathcal{Q}\left(U_{\mathrm{osc}}, V_{\mathrm{QG}}\right) \mid \triangle_{q}^{\mathrm{v}} U_{\mathrm{osc}}\right)\right|+\left|\left(\triangle_{q}^{\mathrm{v}} \mathcal{Q}\left(U_{\mathrm{osc}}, U_{\mathrm{osc}}\right) \mid \triangle_{q}^{\mathrm{v}} U_{\mathrm{osc}}\right)\right| .
\end{aligned}
$$


Taking moreover in account the estimates 8.8 and 8.9 the above inequality turns into

$$
\begin{aligned}
\frac{1}{2} \frac{\mathrm{d}}{\mathrm{d} t}\left\|\triangle_{q}^{\mathrm{v}} U_{\mathrm{osc}}\right\|_{L^{2}\left(\mathbb{T}^{3}\right)}^{2}+c\left\|\triangle_{q}^{\mathrm{v}} \nabla_{h} U_{\mathrm{osc}}\right\|_{L^{2}\left(\mathbb{T}^{3}\right)}^{2} & \leqslant \\
\leqslant & C b_{q}(t) 2^{-2 q s}\left\|\nabla_{h} \Omega\right\|_{H^{0, s}}\left\|\nabla_{h} U_{\mathrm{osc}}\right\|_{H^{0, s}}\left\|U_{\mathrm{osc}}\right\|_{H^{0, s}} \\
& +C b_{q}(t) 2^{-2 q s}\|\Omega\|_{H^{0, s}}^{1 / 2}\left\|\nabla_{h} \Omega\right\|_{H^{0, s}}^{1 / 2}\left\|U_{\mathrm{osc}}\right\|_{H^{0, s}}^{1 / 2}\left\|\nabla_{h} U_{\mathrm{osc}}\right\|_{H^{0, s}}^{3 / 2} \\
& +C b_{q}(t) 2^{-2 q s}\left\|\nabla_{h} U_{\mathrm{osc}}\right\|_{L^{2}\left(\mathbb{T}^{3}\right)}\left\|U_{\mathrm{osc}}\right\|_{H^{0, s}}\left\|\nabla_{h} U_{\mathrm{osc}}\right\|_{H^{0, s}} \\
& +C b_{q}(t) 2^{-2 q s}\left\|U_{\mathrm{osc}}\right\|_{L^{2}\left(\mathbb{T}^{3}\right)}^{1 / 2}\left\|\nabla_{h} U_{\mathrm{osc}}\right\|_{L^{2}\left(\mathbb{T}^{3}\right)}^{1 / 2}\left\|U_{\mathrm{osc}}\right\|_{H^{0, s}}^{1 / 2}\left\|\nabla_{h} U_{\mathrm{osc}}\right\|_{H^{0, s}}^{3 / 2} .
\end{aligned}
$$

We recall that $\left(b_{q}\right)_{q}$ is a $\ell^{1}(\mathbb{Z})$ positive sequence which depends on $\Omega$ and $U_{\text {osc }}$ and such that $\sum_{q} b_{q}(t) \leqslant 1$. Multiplying both sides of 6.4 for $2^{2 q s}$, summing over $q \in \mathbb{Z}$, and using the inequalities $2 a b \leqslant a^{2}+b^{2}$ and $a b \leqslant \frac{1}{4} a^{4}+\frac{3}{4} b^{4 / 3}$ we obtain

$$
\frac{\mathrm{d}}{\mathrm{d} t}\left\|U_{\mathrm{osc}}\right\|_{H^{0, s}}^{2}+c\left\|\nabla_{h} U_{\mathrm{osc}}\right\|_{L^{2}\left(\mathbb{T}^{3}\right)}^{2} \leqslant 2 C\left(\left(1+\|\Omega\|_{H^{0, s}}^{2}\right)\left\|\nabla_{h} \Omega\right\|_{H^{0, s}}^{2}+\left(1+\left\|U_{\mathrm{osc}}\right\|_{L^{2}\left(\mathbb{T}^{3}\right)}^{2}\right)\left\|\nabla_{h} U_{\mathrm{osc}}\right\|_{L^{2}\left(\mathbb{T}^{3}\right)}^{2}\right)\left\|U_{\mathrm{osc}}\right\|_{H^{0, s}}^{2}
$$

applying Gronwall inequality to 6.5 we obtain

$$
\begin{aligned}
& \left\|U_{\mathrm{osc}}(t)\right\|_{H^{0, s}}^{2}+c \int_{0}^{t}\left\|U_{\mathrm{osc}}(\tau)\right\|_{H^{0, s}}^{2} \mathrm{~d} \tau \\
& \quad \leqslant C\left\|U_{\mathrm{osc}, 0}\right\|_{H^{0, s}} \exp \left\{2 C \int_{0}^{t}\left(1+\|\Omega(\tau)\|_{H^{0, s}}^{2}\right)\left\|\nabla_{h} \Omega(\tau)\right\|_{H^{0, s}}^{2}+\left(1+\left\|U_{\mathrm{osc}}(\tau)\right\|_{L^{2}\left(\mathbb{T}^{3}\right)}^{2}\right)\left\|\nabla_{h} U_{\mathrm{osc}}(\tau)\right\|_{L^{2}\left(\mathbb{T}^{3}\right)}^{2} \mathrm{~d} \tau\right\},
\end{aligned}
$$

concluding.

\subsection{Proof of Theorem 1.12}

At this point it is very easy to prove Theorem 1.12 Let us consider a data $V_{0} \in H^{0, s}, \Omega_{0} \in H^{0, s}, s \geqslant 1$ and $V_{0}$ with zero horizontal average. Thanks to Proposition 6.2 we know that $\Omega \in \mathcal{C}\left(\mathbb{R}_{+} ; H^{0, s-1}\right) \cap \mathcal{C}\left(\mathbb{R}_{+} ; H^{0, s}\right), \nabla_{h} \Omega \in L^{2}\left(\mathbb{R}_{+} ; H^{0, s-1}\right) \cap$ $L^{2}\left(\mathbb{R}_{+} ; H^{0, s}\right)$. This implies, thanks to Lemma 5.5 , that $\Lambda_{v}^{s} V_{\mathrm{QG}} \in \mathcal{C}\left(\mathbb{R}_{+} ; L^{2}\left(\mathbb{T}^{3}\right)\right), \nabla_{h} \Lambda_{v}^{s} V_{\mathrm{QG}} \in L^{2}\left(\mathbb{R}_{+} ; L^{2}\left(\mathbb{T}^{3}\right)\right)$. Since $V_{\mathrm{QG}}$ is defined as $V_{\mathrm{QG}}=\Pi_{\mathrm{QG}} U$ where $\Pi_{\mathrm{QG}}$ is a Fourier multiplier of order zero, which maps continuously any $H^{s, s^{\prime}}$ space onto itself, we deduce that $V_{\mathrm{QG}} \in L^{\infty}\left(\mathbb{R}_{+}, L^{2}\right), \nabla_{h} V_{\mathrm{QG}} \in L^{2}\left(\mathbb{R}_{+}, L^{2}\right)$ since $U$ is so thanks to Theorem 1.10 . Hence $V_{\mathrm{QG}} \in \mathcal{C}\left(\mathbb{R}_{+} ; H^{0, s}\right), \nabla_{h} V_{\mathrm{QG}} \in L^{2}\left(\mathbb{R}_{+} ; H^{0, s}\right)$. For the oscillating part it suffices to apply Proposition 6.5 and the proof is complete.

We outline how to prove that solutions to the limit system are $H^{0, s^{\prime}}$-stable, for $s^{\prime} \in[-1 / 2, s)$ globally with a continuous dependence of the initial data. To do so consider the two solutions $U_{1}, U_{2}$ to the limit system

$$
\begin{aligned}
& \left\{\begin{array}{l}
\partial_{t} U_{1}+\mathcal{Q}\left(U_{1}, U_{1}\right)-\mathbb{D} U_{1}=0 \\
\operatorname{div} u_{1}=0 \\
\left.U_{1}\right|_{t=0}=U_{1,0}
\end{array}\right. \\
& \left\{\begin{array}{l}
\partial_{t} U_{2}+\mathcal{Q}\left(U_{2}, U_{2}\right)-\mathbb{D} U_{2}=0 \\
\operatorname{div} u_{2}=0 \\
\left.U_{2}\right|_{t=0}=U_{2,0} .
\end{array}\right.
\end{aligned}
$$

Subtracting (6.7) from [6.6) and setting $U=U_{1}-U_{2}$ we obtain the following system

$$
\left\{\begin{array}{l}
\partial_{t} U+\mathcal{Q}\left(U_{1}, U\right)+\mathcal{Q}\left(U, U_{2}\right)-\mathbb{D} U=0 \\
\operatorname{div} u=0 \\
\left.U\right|_{t=0}=U_{0}=U_{1,0}-U_{2,0} .
\end{array}\right.
$$

The horizontal average of $U$ is zero thanks to the propagation results proved in Section 5.1 hence we can now apply the stability result stated in Proposition 2.9. proved by M. Paicu in [37], to the system 6.8. This gives the following estimate

$$
\begin{aligned}
\|U\|_{H^{0,-\frac{1}{2}}}^{2} & +c \int_{0}^{t}\left\|\nabla_{h} U(\tau)\right\|_{H^{0,-\frac{1}{2}}}^{2} \mathrm{~d} \tau \\
\leqslant C\left\|U_{0}\right\|_{H^{0,-\frac{1}{2}}}^{2} \exp \left\{\int_{0}^{t}\left(1+\|U(\tau)\|_{H^{0, s}}^{2}\right)\left\|\nabla_{h} U(\tau)\right\|_{H^{0, s}}^{2} \mathrm{~d} \tau+\int_{0}^{t}\left(1+\left\|U_{1}(\tau)\right\|_{H^{0, s}}^{2}\right)\left\|\nabla_{h} U_{1}(\tau)\right\|_{H^{0, s}}^{2} \mathrm{~d} \tau\right. & \left.+\int_{0}^{t}\left(1+\left\|U_{2}(\tau)\right\|_{H^{0, s}}^{2}\right)\left\|\nabla_{h} U_{2}(\tau)\right\|_{H^{0, s}}^{2} \mathrm{~d} \tau\right\} .
\end{aligned}
$$


The argument of the exponential is indeed uniformly bounded thanks to the estimates on the limit system performed above, whence if $\left\|U_{0}\right\|_{H^{0,-\frac{1}{2}}}^{2}$ is small the whole right hand side of the above equation if small. Since moreover

$$
\|U\|_{H^{0, s}}^{2}+c \int_{0}^{t}\left\|\nabla_{h} U(\tau)\right\|_{H^{0, s}}^{2} \mathrm{~d} \tau \leqslant C\left(\left\|U_{0}\right\|_{H^{0, s}}^{2}\right)
$$

uniformly in $t$ by interpolation we prove the assertion stated above.

\section{Convergence of the system as $\varepsilon \rightarrow 0$}

Remark 7.1. We point out the fact that Proposition 2.9 can be applied as well to systems of the form

$$
\partial_{t} w+\mathcal{Q}^{\varepsilon}(w, w)+\mathcal{Q}^{\varepsilon}(u, w)-a_{h}(D) w=f, \quad \operatorname{div} w=0 .
$$

Remark 7.2. In the present section our aim is to use Proposition 2.7 and 2.9 to the systems $\left[\mathrm{FS}_{\varepsilon}\right.$ ] and $[\mathrm{S}$. Let us compare these two systems with $\left[\mathrm{NS}_{h}\right)$ : the only structural difference between these two is that in $\left[\mathrm{FS}_{\varepsilon}\right.$ and $[\mathrm{S}$ the Poincaré semigroup couples velocity field and temperature $v^{\varepsilon}, T^{\varepsilon}$ in a new variable $U^{\varepsilon}$, but the structure itself of the equation is unchanged. For this reason Propositions 2.7 and 2.9 can be applied in the present case.

We shall require as well the following result

Lemma 7.3. Let $f \in H^{s, s^{\prime}}, s, s^{\prime} \in \mathbb{R}$ such that the horizontal average $\underline{f} \in H_{v}^{s^{\prime}}$. Than

$$
\|f\|_{H_{v}^{s^{\prime}}} \leqslant\|f\|_{H^{s, s^{\prime}}}
$$

Proof. Since the element $\underline{f}$ is the horizontal average of the function $f$ we can indeed argue that

$$
\underline{f}\left(x_{3}\right)=\mathcal{F}_{v}^{-1}\left(\left(\hat{f}\left(0, n_{3}\right)\right)_{n_{3}}\right)
$$

at least in $L^{2}$. Whence calculating explicitly the Sobolev norms

$$
\begin{aligned}
\|\underline{f}\|_{H_{v}^{s^{\prime}}}^{2} & =\sum_{n_{3} \in \mathbb{Z}}\left(1+n_{3}^{2}\right)^{s^{\prime}}\left|\hat{f}\left(0, n_{3}\right)\right|^{2} \\
\|f\|_{H^{s, s^{\prime}}}^{2} & =\sum_{n \in \mathbb{Z}^{3}}\left(1+\left|n_{h}\right|^{2}\right)^{s}\left(1+n_{3}^{2}\right)^{s^{\prime}}\left|\hat{f}\left(n_{h}, n_{3}\right)\right|^{2},
\end{aligned}
$$

Comparing the expressions in 7.1 and 7.2 we remark that 7.1 is the restriction of 7.2 onto the fiber $\left\{n_{h}=0\right\}$, concluding.

Remark 7.4. Let us recall that Theorem 1.4 implies that, for each $\varepsilon>0$ fixed, there exists a maximal time $T_{\varepsilon}^{\star} \leqslant \infty$ such that for each $T^{\star}<T_{\varepsilon}^{\star}$ and $s>1 / 2$ the function $U^{\varepsilon}$ belongs to the space

$$
U^{\varepsilon} \in L^{\infty}\left(\left[0, T^{\star}\right] ; H^{0, s}\right), \quad \nabla_{h} U^{\varepsilon} \in L^{2}\left(\left[0, T^{\star}\right] ; H^{0, s}\right) .
$$

We prove that, given $V_{0}^{\varepsilon} \in H^{0, s}, s>1$, the solution of the filtered system $\left[\mathrm{FS}_{\varepsilon}\right.$ converges to the solution of the limit system [5.9], (5.10) in the sense that

$$
\begin{aligned}
\lim _{\varepsilon \rightarrow 0}\left(V^{\varepsilon}-\mathcal{L}\left(\frac{t}{\varepsilon}\right) U\right) & =0 & & \text { in } \mathcal{C}\left(\mathbb{R}_{+} ; H^{0, \sigma}\right) \\
\lim _{\varepsilon \rightarrow 0} \nabla_{h}\left(V^{\varepsilon}-\mathcal{L}\left(\frac{t}{\varepsilon}\right) U\right) & =0 & & \text { in } L^{2}\left(\mathbb{R}_{+} ; H^{0, \sigma}\right)
\end{aligned}
$$

for $\sigma \in[1, s)$, where $U=U_{\mathrm{osc}}+U_{\mathrm{QG}}$ and $U_{\mathrm{QG}}=V_{\mathrm{QG}}=\left(\begin{array}{c}\nabla_{h}^{\perp} \\ 0 \\ -F \partial_{3}\end{array}\right) \Delta_{F}^{-1} \Omega$ with $\Omega$ solution of [5.9]. To do so we use a method introduced by S. Schochet in [39] in the framework of hyperbolic systems. A suitable change of variable has to be performed so that the singular perturbations cancel among themselves. The same method has been studied in a wide generality by I. Gallagher in [24] in the generic context of parabolic (nonlinear) equations with singular, linear, skew-symmetric perturbation. We mention as well the works [26] and [35] in which such technique has been used. 
We want to underline a major difference between the application of Schochet method in the present work and in the work [35]. In [35] in fact the convergence takes place for the values of $\sigma$ between $1 / 2$ and $s$. In the present case $\sigma \in[1, s)$, this difference is motivated by the fact that the limit system $\left[\mathrm{S}\right.$ is globally well posed in $H^{0, s}, s>1$ only. This is due to the fact that we proved the propagation of $H^{0, s}, s>0$ data for $\Omega$ in Proposition 6.2. hence we applied Lemma 5.5 to state that $H^{0, s}, s>1$ data is propagated for $V_{\mathrm{QG}}$.

Let us denote $T_{\varepsilon}^{\star}$ the maximal lifespan of $U^{\varepsilon}$ solution of $\left[\overline{\mathrm{FS}_{\varepsilon}}\right]$ in the space $H^{0, s}\left(\mathbb{T}^{3}\right)$ with $s>1$, which exists thanks to the work 37. Then there exists a time $T_{\varepsilon}^{\star} \geqslant T>0$ such that $U^{\varepsilon} \in \mathcal{C}\left([0, T] ; H^{0, s}\right)$ and $\nabla_{h} U^{\varepsilon} \in L^{2}\left([0, T] ; H^{0, s}\right)$ uniformly in $\varepsilon$ small enough. Let us define $W^{\varepsilon}=U^{\varepsilon}-U$ defined on the interval $\left[0, T_{\varepsilon}^{\star}\right]$ taking values in $H^{0, s}$. The difference $W^{\varepsilon}$ satisfies the following equation

$$
\left\{\begin{array}{l}
\partial_{t} W^{\varepsilon}+\mathcal{Q}^{\varepsilon}\left(W^{\varepsilon}, W^{\varepsilon}\right)+\widetilde{\mathcal{Q}}^{\varepsilon}\left(U, W^{\varepsilon}\right)-\mathbb{D}^{\varepsilon} W^{\varepsilon} \\
\quad=-\left(\mathbb{D}^{\varepsilon}-\mathbb{D}\right) U-\left(\mathcal{Q}^{\varepsilon}(U, U)-\mathcal{Q}(U, U)\right) \\
\operatorname{div} w^{\varepsilon}=0 \\
\left.W^{\varepsilon}\right|_{t=0}=0
\end{array}\right.
$$

where the form $\tilde{\mathcal{Q}}^{\varepsilon}$ is symmetric, bilinear and defined via

$$
\widetilde{\mathcal{Q}}^{\varepsilon}(A, B)=\mathcal{Q}^{\varepsilon}(A, B)+\mathcal{Q}^{\varepsilon}(B, A)
$$

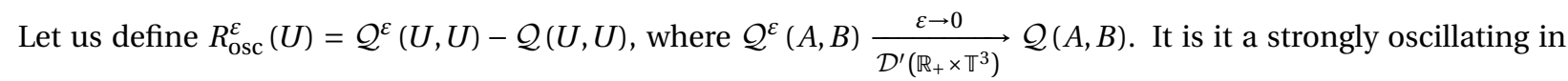
time function, given by the formula

$$
R_{\mathrm{osc}}^{\varepsilon}(U)=\mathcal{F}^{-1}\left(\sum_{\substack{\omega_{k, b, c}^{a, b-n} \neq 0 \\ 1 \leqslant j \leqslant 3}} e^{i \frac{t}{\varepsilon} \omega_{k, n-k, n}^{a, b, c}}\left(U^{a, j}(k)\left(n_{j}-k_{j}\right) U^{b}(n-k) \mid e^{c}(n)\right)_{\mathbb{C}^{4}} e^{c}(n)\right),
$$

where we used the notation $\omega_{k, n-k, n}^{a, b, c}=\omega^{a}(k)+\omega^{b}(n-k)-\omega^{c}(n), a, b, c \in\{ \pm\}, \omega^{ \pm}(n)$ defined as in 3.4), $U^{a}(k)=$ $\left(\hat{U}(k) \mid e^{a}(k)\right) e^{a}(k)$ and $U^{a, j}$ is the $j$-th component of $U^{a}$.

As well the function $S_{\mathrm{osc}}^{\varepsilon}=\left(\mathbb{D}^{\varepsilon}-\mathbb{D}\right) U$ is a highly oscillating function defined as

$$
S_{\mathrm{osc}}^{\varepsilon}(U)=\mathcal{F}^{-1}\left(\sum_{\omega_{n}^{a, b} \neq 0} e^{i \frac{t}{\varepsilon} \omega_{n}^{a, b}}\left(\mathbf{D}(n) U^{b}(n) \mid e^{a}(n)\right)_{\mathbb{C}^{4}} e^{a}(n)\right),
$$

and as well as $R_{\mathrm{osc}}^{\varepsilon}, S_{\mathrm{osc}}^{\varepsilon} \rightarrow 0$ as $\varepsilon \rightarrow 0$ in $\mathcal{D}^{\prime}$ only. For the rest of the section $(\cdot \mid \cdot)$ denotes $(\cdot \mid \cdot)_{\mathbb{C}^{4}}$.

We decompose $R_{\text {osc }}^{\varepsilon}$ and $S_{\text {osc }}^{\varepsilon}$ in high and low frequencies, i.e.

$$
\begin{aligned}
& R_{\mathrm{osc}, \mathrm{LF}}^{\varepsilon, N}(U)=\mathcal{F}^{-1}\left(1_{\{|n| \leqslant N\}} \sum_{\substack{a, b, c \\
\omega_{,, n-k, n} \neq 0 \\
1 \leqslant j \leqslant 3}} e^{i \frac{t}{\varepsilon} \omega_{k, n-k, n}^{a, b, c}} 1_{\{|k| \leqslant N\}}\left(U^{a, j}(k)\left(n_{j}-k_{j}\right) U^{b}(n-k) \mid e^{c}(n)\right) e^{c}(n)\right), \\
& S_{\mathrm{osc}, \mathrm{LF}}^{\varepsilon, N}(U)=\mathcal{F}^{-1}\left(1_{\{|n| \leqslant N\}} \sum_{\omega_{n}^{a, b} \neq 0} e^{i \frac{t}{\varepsilon} \omega_{n}^{a, b}}\left(\mathbf{D}(n) U^{b}(n) \mid e^{a}(n)\right) e^{a}(n)\right),
\end{aligned}
$$

and

$$
\begin{aligned}
& R_{\mathrm{osc}, \mathrm{HF}}^{\varepsilon, N}(U)=R_{\mathrm{osc}}^{\varepsilon}(U)-R_{\mathrm{osc}, \mathrm{LF}}^{\varepsilon, N}(U) \\
& S_{\mathrm{osc}, \mathrm{HF}}^{\varepsilon, N}(U)=S_{\mathrm{osc}}^{\varepsilon}(U)-S_{\mathrm{osc}, \mathrm{LF}}^{\varepsilon, N) .}
\end{aligned}
$$

Indeed the subscript $f_{\mathrm{HF}}$ stands for high frequencies and the subscript $f_{\mathrm{LF}}$ stands for low frequencies.

Concerning the high frequencies terms the following lemma hold

Lemma 7.5. If $N \rightarrow \infty$ the terms $R_{\mathrm{osc}, \mathrm{HF}}^{\varepsilon, N}(U), S_{\mathrm{osc}, \mathrm{HF}}^{\varepsilon, N}(U)$ tend uniformly to 0 in $\varepsilon$ respectively in the space $L^{p}\left([0, T] ; H^{-1,-1 / 2}\right)$ and $L^{p}\left([0, T] ; H^{-1, s}\right)$ for all $1 \leqslant p \leqslant 2, s>1$.

The proof of Lemma 7.5 is postponed to the end of the section for the sake of clarity. 
The term $R_{\mathrm{osc}, \mathrm{LF}}^{\varepsilon, N}(U)$ tends only weakly to zero. In order to absorb it in the following computations we introduce the following notation

$$
\begin{aligned}
& \widetilde{R}_{\mathrm{osc}, \mathrm{LF}}^{\varepsilon, N}(U)=\mathcal{F}^{-1}\left(1_{\{|n| \leqslant N\}} \sum_{\substack{\omega_{k, n-k, n}^{a, b} \neq 0 \\
1 \leqslant j \leqslant 3}} \frac{e^{i \frac{t}{\varepsilon} \omega_{k, n-k, n}^{a, b, c}}}{\omega_{k, n-k, n}^{a, b, c}} 1_{\{|n| \leqslant N\}}\left(U^{a, j}(t, k)\left(n_{j}-k_{j}\right) U^{b}(t, n-k) \mid e^{c}(n)\right) e^{c}(n)\right) \\
& \widetilde{S}_{\mathrm{osc}, \mathrm{LF}}^{\varepsilon, N}(U)=\mathcal{F}^{-1}\left(1_{\{|n| \leqslant N\}} \sum_{\omega_{n}^{a, b} \neq 0} \frac{e^{i \frac{t}{\varepsilon} \omega_{n}^{a, b}}}{i \omega_{n}^{a, b}}\left(\mathbf{D}(n) U^{b}(n) \mid e^{a}(n)\right) e^{a}(n)\right)
\end{aligned}
$$

We define as well the following auxiliary unknown

$$
\Psi_{\mathrm{LF}}^{\varepsilon, N}=W^{\varepsilon}+\varepsilon\left(\widetilde{R}_{\mathrm{osc}, \mathrm{LF}}^{\varepsilon, N}(U)+\widetilde{S}_{\mathrm{osc}, \mathrm{LF}}^{\varepsilon, N}(U)\right) .
$$

Considering the new unknown defined in 7.4 into 7.3 , after some algebraic manipulation, we obtain that $\Psi_{\mathrm{LF}}^{\varepsilon, N}$ satisfies the following equation

$$
\partial_{t} \Psi_{\mathrm{LF}}^{\varepsilon, N}+\frac{1}{2} \widetilde{\mathcal{Q}}\left(\Psi_{\mathrm{LF}}^{\varepsilon, N}, \Psi_{\mathrm{LF}}^{\varepsilon, N}-2 \varepsilon\left(\widetilde{R}_{\mathrm{osc}, \mathrm{LF}}^{\varepsilon, N}(U)+\widetilde{S}_{\mathrm{osc}, \mathrm{LF}}^{\varepsilon, N}(U)\right)+2 U\right)-\mathbb{D}^{\varepsilon} \Psi_{\mathrm{LF}}^{\varepsilon, N}=\Gamma^{\varepsilon, N}(U),
$$

where

and

$$
\Gamma^{\varepsilon, N}=R_{\mathrm{osc}, \mathrm{HF}}^{\varepsilon, N}+S_{\mathrm{osc}, \mathrm{HF}}^{\varepsilon, N}+\varepsilon \Gamma_{N}^{\varepsilon}
$$

$$
\begin{aligned}
\Gamma_{N}^{\varepsilon}=\mathbb{D}^{\varepsilon}\left(\widetilde{R}_{\mathrm{osc}, \mathrm{LF}}^{\varepsilon, N}(U)\right. & \left.+\widetilde{S}_{\mathrm{osc}, \mathrm{LF}}^{\varepsilon, N}(U)\right) \\
& +\frac{1}{2} \widetilde{\mathcal{Q}}\left(\left(\widetilde{R}_{\mathrm{osc}, \mathrm{LF}}^{\varepsilon, N}(U)+\widetilde{S}_{\mathrm{osc}, \mathrm{LF}}^{\varepsilon, N}(U)\right), \varepsilon\left(\widetilde{R}_{\mathrm{osc}, \mathrm{LF}}^{\varepsilon, N}(U)+\widetilde{S}_{\mathrm{osc}, \mathrm{LF}}^{\varepsilon, N}(U)\right)-2 U\right)+\left(\widetilde{R}_{\mathrm{osc}, \mathrm{LF}}^{\varepsilon, N, t}(U)+\widetilde{S}_{\mathrm{osc}, \mathrm{LF}}^{\varepsilon, N, t}(U)\right)
\end{aligned}
$$

and respectively

$$
\begin{aligned}
& \widetilde{R}_{\mathrm{osc}, \mathrm{LF}}^{\varepsilon, N, t}=\mathcal{F}^{-1}\left(1_{\{|n| \leqslant N\}} \sum_{\substack{\omega_{k, n-k, n}^{a, b, c} \\
1 \leqslant j \leqslant 3}} \frac{e^{i \frac{t}{\varepsilon} \omega_{k, n-k, n}^{a, b, c}}}{\omega_{k, n-k, n}^{a, b, c}} 1_{\{|n| \leqslant N\}} \partial_{t}\left[\left(U^{a, j}(t, k)\left(n_{j}-k_{j}\right) U^{b}(t, n-k) \mid e^{c}(n)\right) e^{c}(n)\right]\right) \\
& \widetilde{S}_{\mathrm{osc}, \mathrm{LF}}^{\varepsilon, N, t}=\mathcal{F}^{-1}\left(1_{\{|n| \leqslant N\}} \sum_{\omega_{n}^{a, b} \neq 0} \frac{e^{i \frac{t}{\varepsilon} \omega_{n}^{a, b}}}{i \omega_{n}^{a, b}} \partial_{t}\left[\left(\mathbf{D}(n) U^{b}(t, n) \mid e^{a}(n)\right) e^{a}(n)\right]\right) .
\end{aligned}
$$

Lemma 7.6. The term $\Gamma_{N}^{\varepsilon}$ given by the relation $(7.6$ is bounded uniformly in $\varepsilon$ by a constant $C(N)$, which depend on $N$ solely, in the spaces $L^{p}\left([0, T] ; H^{-1,-1 / 2}\right)$ for $1 \leqslant p \leqslant 2$.

Proof. Since the functions considered are localized in a ball of radius $N$ in the frequency space, it is possible to gain all the regularity that wanted at the price of a constant which behaves like a power of $N$. Hence, if $\omega_{n}^{a, b}, \omega_{k, n-k, n}^{a, b, c} \neq 0$,

$$
\frac{1}{\left|\omega_{n}^{a, b}\right|}, \frac{1}{\left|\omega_{k, n-k, n}^{a, b, c}\right|} \leqslant C(N) .
$$

Whence we easily obtain that $\Gamma_{N}^{\varepsilon}$ belongs to the space $L^{p}\left(\mathbb{R}_{+}, H^{-1,-1 / 2}\right)$ and that is uniformly bounded by a constant $C(N)$.

We remark that for $\varepsilon$ sufficiently small the term $U-\varepsilon\left(\widetilde{R}_{\mathrm{osc}, \mathrm{LF}}^{\varepsilon, N}(U)+\widetilde{S}_{\mathrm{osc}, \mathrm{LF}}^{\varepsilon, N}(U)\right)=\Psi_{\mathrm{LF}}^{\varepsilon, N}$ has a small horizontal mean in $H_{v}^{s}$, whence we can apply Proposition 2.9 to equation $(7.5)$ in order to obtain, for all $t \in\left[0, T_{\varepsilon}^{\star}\right]$, the following bound

$$
\begin{aligned}
\left\|\Psi_{\mathrm{LF}}^{\varepsilon, N}(t)\right\|_{H^{0,-1 / 2}}^{2}+c \int_{0}^{t} & \left\|\nabla_{h} \Psi_{\mathrm{LF}}^{\varepsilon, N}(\tau)\right\|_{H^{0,-1 / 2}}^{2} \mathrm{~d} \tau \leqslant \mathcal{C}\left(\left\|U_{0}\right\|_{H^{0, s_{0}}}\right) \\
\times & \left.\times\left\|\Psi_{\mathrm{LF}}^{\varepsilon, N}(0)\right\|_{H^{0,-1 / 2}}^{2}+\int_{0}^{t}\left\|\Gamma^{\varepsilon, N}(\tau)\right\|_{H^{-1,-1 / 2}} \mathrm{~d} \tau+\int_{0}^{t}\left\|\Gamma^{\varepsilon, N}(\tau)\right\|_{H^{-1,-1 / 2}}^{2} \mathrm{~d} \tau\right) \\
& \times \exp \left\{\int_{0}^{t}\left\|\Gamma^{\varepsilon, N}(\tau)\right\|_{H^{-1,-1 / 2}} \mathrm{~d} \tau+\int_{0}^{t}\left(1+\left\|\Psi_{\mathrm{LF}}^{\varepsilon, N}(\tau)\right\|_{H^{0, s_{0}}}^{2}\right)\left\|\nabla_{h} \Psi_{\mathrm{LF}}^{\varepsilon, N}(\tau)\right\|_{H^{0, s_{0}}}^{2} \mathrm{~d} \tau\right\} .
\end{aligned}
$$

Since we want to obtain global in time solutions it is important to have $\Gamma^{\mathcal{E}, N}$ at the same time in both spaces $L^{1}\left(\mathbb{R}_{+} ; H^{-1,-1 / 2}\right)$ and $L^{2}\left(\mathbb{R}_{+} ; H^{-1,-1 / 2}\right)$. 
- We remark the fact that writing the estimate 7.7 we used implicitly the bound

$$
\int_{0}^{t}\left(1+\|U(\tau)\|_{H^{0, s_{0}}}^{2}\right)\left\|\nabla_{h} U(\tau)\right\|_{H^{0, s_{0}}}^{2} \mathrm{~d} \tau \leqslant \frac{C}{c} \widetilde{\mathcal{C}}\left(\left\|U_{0}\right\|_{H^{0, s_{0}}}\right),
$$

for $s_{0}>1$, and we denoted $\mathcal{C}\left(\left\|U_{0}\right\|_{H^{H^{0, s_{0}}}}\right)=\exp \left\{\frac{C}{c} \widetilde{\mathcal{C}}\left(\left\|U_{0}\right\|_{H^{0, s_{0}}}\right)\right\}$.

- We used Lemma 7.3 to deduce the inequality

$$
\left\|\underline{\Gamma}^{\mathcal{E}, N}(\tau)\right\|_{H_{v}^{-1 / 2}} \leqslant\left\|\Gamma^{\mathcal{E}, N}(\tau)\right\|_{H^{-1,-1 / 2}},
$$

which has consequently be applied in order to deduce [7.7.

Considering Lemma 7.5 we can say that for each $\eta>0$ there exits a large enough $N$ such that, setting $\mathcal{X}=L^{1}\left(\mathbb{R}_{+} ; H^{-1,-1 / 2}\right) \cap$ $L^{2}\left(\mathbb{R}_{+} ; H^{-1,-1 / 2}\right)$,

$$
\left\|R_{\mathrm{osc}, \mathrm{HF}}^{\varepsilon, N}+S_{\mathrm{osc}, \mathrm{HF}}^{\varepsilon, N}\right\|_{\mathcal{X}} \leqslant \frac{\eta}{2}
$$

and thanks to Lemma 7.6 for $\varepsilon$ sufficiently small

$$
\varepsilon\left\|\Gamma_{N}^{\varepsilon}\right\|_{\mathcal{X}} \leqslant \varepsilon C(N) \leqslant \frac{\eta}{2}
$$

whence we obtain that

$$
\left\|\Gamma^{\mathcal{E}, N}\right\|_{\mathcal{X}} \leqslant \eta
$$

Thanks to the definition 7.4 we can argue that for each $\eta>0$ and $t<T$ time of local existence of the solutions, there exists a $\varepsilon_{1}=\varepsilon_{1}(\eta, T)$ such that for each $\varepsilon \in\left(0, \varepsilon_{1}\right)$ :

$$
\left\|\Psi_{\mathrm{LF}}^{\varepsilon, N}(t)-W^{\varepsilon}(t)\right\|_{H^{0,-1 / 2}}^{2}+c \int_{0}^{t}\left\|\nabla_{h} \Psi_{\mathrm{LF}}^{\varepsilon, N}(\tau)-\nabla_{h} W^{\varepsilon}(\tau)\right\|_{H^{0,-1 / 2}}^{2} \mathrm{~d} \tau \leqslant \varepsilon C(N) \leqslant \frac{\eta}{2},
$$

in the same way we can write

$$
\left\|\Psi_{\mathrm{LF}}^{\varepsilon, N}(0)\right\|_{H^{0,-1 / 2}}=\varepsilon\left\|\widetilde{R}_{\mathrm{osc}, \mathrm{LF}}^{\varepsilon, N}\left(U_{0}\right)+\widetilde{S}_{\mathrm{osc}, \mathrm{LF}}^{\varepsilon, N}\left(U_{0}\right)\right\|_{H^{0,-1 / 2}} \leqslant \varepsilon C(N)\left\|U_{0}\right\|_{H^{0, s}}^{2} \leqslant \frac{\eta}{2} .
$$

Whence for $\varepsilon$ sufficiently small and $t \in\left[0, T_{\varepsilon}^{\star}\right)$ we have

$$
\left\|\Psi_{\mathrm{LF}}^{\varepsilon, N}(t)\right\|_{H^{0,-1 / 2}}^{2}+c \int_{0}^{t}\left\|\nabla_{h} \Psi_{\mathrm{LF}}^{\varepsilon, N}(\tau)\right\|_{H^{0,-1 / 2}}^{2} \mathrm{~d} \tau \leqslant C \eta\left(1+\exp \left\{\int_{0}^{t}\left\|\nabla_{h} \Psi_{\mathrm{LF}}^{\varepsilon, N}(\tau)\right\|_{H^{0, s_{0}}}^{2}\left(1+\left\|\Psi_{\mathrm{LF}}^{\varepsilon, N}(\tau)\right\|_{H^{0, s_{0}}}^{2}\right) \mathrm{d} \tau\right\}\right) .
$$

We use now the definition of $\Psi_{\mathrm{LF}}^{\varepsilon, N}$ given in 7.4 This implies that $\left\|\Psi_{\mathrm{LF}}^{\varepsilon, N}\right\|=\left\|W^{\varepsilon}\right\|+\mathcal{O}_{N}(\varepsilon)$ for $N$ fixed. This means that $\Psi_{\mathrm{LF}}^{\varepsilon, N}$ and $W^{\varepsilon}$ have the same norm up to an error which is comparable to $\varepsilon$ which is, anyway, considered to be small. Whence 7.8 allows us to deduce that

$$
\left\|W^{\varepsilon}(t)\right\|_{H^{0,-1 / 2}}^{2}+c \int_{0}^{t}\left\|\nabla_{h} W^{\varepsilon}(\tau)\right\|_{H^{0,-1 / 2}}^{2} \mathrm{~d} \tau \leqslant C \eta\left(1+\exp \left\{\int_{0}^{t}\left\|\nabla_{h} W^{\varepsilon}(\tau)\right\|_{H^{0, s_{0}}}^{2}\left(1+\left\|W^{\varepsilon}(\tau)\right\|_{H^{0, s_{0}}}^{2}\right) \mathrm{d} \tau\right\}\right) .
$$

For the real numbers $s^{\prime} \in[-1 / 2, s]$ we introduce the following continuous function

$$
f_{\varepsilon, s^{\prime}}(t)=\left\|W^{\varepsilon}(t)\right\|_{H^{0, s^{\prime}}}^{2}+\int_{0}^{t}\left(1+\left\|W^{\varepsilon}(\tau)\right\|_{H^{0, s^{\prime}}}^{2}\right)\left\|\nabla_{h} W^{\varepsilon}(\tau)\right\|_{H^{0, s^{\prime}}}^{2} \mathrm{~d} \tau .
$$

The function $\left\|W^{\varepsilon}(t)\right\|_{H^{0, s^{\prime}}}^{2}$ is defined on the interval $\left[0, T_{\varepsilon}^{\star}\right)$, by use of $(7.9]$ we get

$$
f_{\varepsilon,-1 / 2}(t) \leqslant C \eta
$$

for each $t \in\left[0, T_{\varepsilon}^{\star}\right)$.

We consider now an $s_{0}>1$ and the maximal time

$$
T_{\varepsilon}^{s_{0}}=\sup \left\{0<t<T_{\varepsilon}^{\star} \mid f_{\varepsilon, s_{0}}(t) \leqslant 1 \text {, for each } 0 \leqslant t \leqslant T_{\varepsilon}^{s_{0}}\right\} .
$$

Interpolating between $H^{0,-1 / 2}$ and $H^{0, s_{0}}$ we get

$$
f_{\varepsilon, \sigma}(t)=\mathcal{O}\left(\eta^{\vartheta\left(s_{0}, \sigma\right)}\right) \leqslant 1, \quad t \in\left[0, T_{\varepsilon}^{s_{0}}\right),
$$


where $0<\vartheta\left(s_{0}, \sigma\right) \stackrel{\sigma \rightarrow s_{0}}{\longrightarrow} 0$ and $0<\sigma \in\left[-1 / 2, s_{0}\right)$.

We consider at this point $U^{\varepsilon}=W^{\varepsilon}+U$, since $U$ has zero horizontal mean we can easily point out that

$$
\underline{U^{\varepsilon}}(t)=\underline{W^{\varepsilon}}(t) .
$$

Whence using Lemma 7.3 the definition of the function $f_{\varepsilon, \sigma}$ given in $(7)$, and the smallness property on $f_{\varepsilon, \sigma}$ given in 7.11) we deduce:

$$
\begin{aligned}
\left\|\underline{W^{\varepsilon}}(t)\right\|_{H_{v}^{\sigma}} & \leqslant\left\|W^{\varepsilon}(t)\right\|_{H^{0, \sigma}}, \\
& \leqslant C \sqrt{f_{\varepsilon, \sigma}(t)}, \\
& \leqslant C \eta^{\vartheta / 2} \ll 1
\end{aligned}
$$

Since the horizontal average of $\underline{U^{\varepsilon}}$ is small we can infer via Proposition 2.7 obtaining, for $\sigma \in\left(1, s_{0}\right)$;

$$
\left\|U^{\varepsilon}(t)\right\|_{H^{0, s}}^{2}+c \int_{0}^{t}\left\|\nabla_{h} U^{\varepsilon}(\tau)\right\|_{H^{0, s}}^{2} \mathrm{~d} \tau \leqslant C\left\|V_{0}\right\|_{H^{0, s}} \exp \left(\int_{0}^{t}\left(1+\left\|U^{\varepsilon}(\tau)\right\|_{H^{0, \sigma}}^{2}\right)\left\|\nabla_{h} U^{\varepsilon}(\tau)\right\|_{H^{0, \sigma}}^{2} \mathrm{~d} \tau\right) .
$$

If $0 \leqslant t<T_{\varepsilon}^{s_{0}}$, and since $U^{\varepsilon}=W^{\varepsilon}+U$ we deduce

$$
\int_{0}^{t}\left(1+\left\|U^{\varepsilon}(\tau)\right\|_{H^{0, \sigma}}^{2}\right)\left\|\nabla_{h} U^{\varepsilon}(\tau)\right\|_{H^{0, \sigma}}^{2} \mathrm{~d} s \leqslant f_{\varepsilon, \sigma}(t)+\int_{0}^{t}\left(1+\|U(\tau)\|_{H^{0, \sigma}}^{2}\right)\left\|\nabla_{h} U(\tau)\right\|_{H^{0, \sigma}}^{2} \mathrm{~d} \tau+F_{\sigma}(t) .
$$

$F_{\sigma}(t)$ in particular is defined as

$$
\begin{aligned}
F_{\sigma}(t) & =\int_{0}^{t}\left(1+\left\|W^{\varepsilon}(\tau)\right\|_{H^{0, \sigma}}^{2}\right)\left\|\nabla_{h} U(\tau)\right\|_{H^{0, \sigma}}^{2} \mathrm{~d} \tau+\int_{0}^{t}\left(1+\|U(\tau)\|_{H^{0, \sigma}}^{2}\right)\left\|\nabla_{h} W^{\varepsilon}(\tau)\right\|_{H^{0, \sigma}}^{2} \mathrm{~d} \tau \\
& \lesssim \int_{0}^{t}\left(1+\left\|W^{\varepsilon}(\tau)\right\|_{H^{0, \sigma}}^{2}\right)\left\|\nabla_{h} U(\tau)\right\|_{H^{0, \sigma}}^{2} \mathrm{~d} \tau+\left(1+\|U\|_{L^{\infty}\left(\mathbb{R}_{+} ; H^{0, \sigma}\right)}^{2}\right) f_{\varepsilon, \sigma}(t) \\
& \lesssim\left(\sup _{[0, t]} f_{\varepsilon, \sigma}\right)\left\|\nabla_{h} U\right\|_{L^{2}\left(\mathbb{R}_{+} ; H^{0, \sigma}\right)}+\left(1+\|U\|_{L^{\infty}\left(\mathbb{R}_{+} ; H^{0, \sigma}\right)}^{2}\right) f_{\varepsilon, \sigma}(t),
\end{aligned}
$$

which in turn implies that, considering the above estimate in 7.13 ,

$$
\begin{aligned}
\int_{0}^{t}\left(1+\left\|U^{\varepsilon}(\tau)\right\|_{H^{0, \sigma}}^{2}\right)\left\|\nabla_{h} U^{\varepsilon}(\tau)\right\|_{H^{0, \sigma}}^{2} \mathrm{~d} s \leqslant & f_{\varepsilon, \sigma}(t)+\int_{0}^{t}\left(1+\|U(\tau)\|_{H^{0, \sigma}}^{2}\right)\left\|\nabla_{h} U(\tau)\right\|_{H^{0, \sigma}}^{2} \mathrm{~d} \tau \\
& +\left(\sup _{[0, t]} f_{\varepsilon, \sigma}\right)\left\|\nabla_{h} U\right\|_{L^{2}\left(\mathbb{R}_{+} ; H^{0, \sigma}\right)}+\left(1+\|U\|_{L^{\infty}\left(\mathbb{R}_{+} ; H^{0, \sigma}\right)}^{2}\right) f_{\varepsilon, \sigma}(t) .
\end{aligned}
$$

We have seen though that in $\left[0, T_{\varepsilon}^{s_{0}}\right)$ that $f_{\varepsilon, \sigma}(t) \leqslant 1$ for $\sigma \in\left(-1 / 2, s_{0}\right)$. Since $U \in L^{\infty}\left(\mathbb{R}_{+}, H^{0, \sigma}\right)$ and $\nabla_{h} U \in L^{2}\left(\mathbb{R}_{+}, H^{0, \sigma}\right)$ for $\sigma \in\left(1, s_{0}\right]$ (this is simply Proposition 6.2 combined with Lemma 5.5 , we obtain that

$$
\int_{0}^{t}\left(1+\left\|U^{\varepsilon}(\tau)\right\|_{H^{0, \sigma}}^{2}\right)\left\|\nabla_{h} U^{\varepsilon}(\tau)\right\|_{H^{0, \sigma}}^{2} \mathrm{~d} s \leqslant C .
$$

If we consider the above bound in 7.12 we deduced that

$$
\left\|U^{\varepsilon}(t)\right\|_{H^{0, s}}^{2}+c \int_{0}^{t}\left\|\nabla_{h} U^{\varepsilon}(s)\right\|_{H^{0, s}}^{2} \mathrm{~d} s \leqslant C,
$$

for all times $t \in\left[0, T_{\varepsilon}^{s_{0}}\right)$ and $s>1$. We deduce that $T_{\varepsilon}^{s_{0}}=T_{\varepsilon}^{\star}$ and since the constant $C$ is independent of the time $t$, this implies that $U^{\varepsilon}(t)$ can be extended in $H^{0, s}$ beyond $T_{\varepsilon}^{\star}$ and hence we obtain that $T_{\varepsilon}^{\star}=\infty$ as long as $\varepsilon$ is sufficiently small. Recalling that $\left\|W^{\varepsilon}\right\|=o(1)$ in $\left[0, T_{\varepsilon}^{\star}\right)$ we deduce that $U^{\varepsilon} \rightarrow U$ globally in time in $H^{0, \sigma}$ for $-1 / 2 \leqslant \sigma<s$.

Proof of Lemma 7.5 . In the following the index $s$ denoting the anisotropic Sobolev space $H^{0, s}$ is always considered to be $s>1$. An interesting feature is that if $s>1 / 2$ then $H_{v}^{s}$ is a Banach algebra. We use this property all along the proof. We perform at first the estimates for the term $R_{\mathrm{osc}, \mathrm{HF}}^{\varepsilon, N}$. Since $U(t)$ is of zero horizontal average for all $t>0$ and $\nabla_{h} U \in L^{2}\left(\mathbb{R}_{+} ; H^{0, s}\right)$ we obtain that $U \in L^{2}\left(\mathbb{R}_{+} ; H^{0, s}\right)$. Consequently $U \in \mathcal{C}\left(\mathbb{R}_{+} ; H^{0, s}\right) \cap L^{2}\left(\mathbb{R}_{+} ; H^{0, s}\right)$, and, interpolating $U \in L^{p^{\prime}}\left(\mathbb{R}_{+}, H^{0, s}\right)$ for each $p^{\prime} \in[2, \infty]$.

Let us observe that the term $R_{\mathrm{osc}, \mathrm{HF}}^{\varepsilon, N}$ can be decomposed as

$$
R_{\mathrm{osc}, \mathrm{HF}}^{\varepsilon, N}=R_{\mathrm{osc}, 1}^{\varepsilon, N}+R_{\mathrm{osc}, 2}^{\varepsilon, N}
$$


where we denoted

$$
R_{\mathrm{osc}, 1}^{\varepsilon, N}=\mathcal{F}^{-1}\left(1_{\{|n| \geqslant N\}} R_{\mathrm{osc}, \mathrm{HF}}^{\varepsilon, N}\right)
$$

and

$$
R_{\mathrm{osc}, 2}^{\varepsilon, N}(U)=\mathcal{F}^{-1}\left(1_{\{|n| \leqslant N\}} \sum_{\substack{\omega_{k, n-k, n}^{a, b, c} \\ 1 \leqslant j \leqslant 3}} e^{i \frac{t}{\varepsilon} \omega_{k, n-k, n}^{a, b, c}} 1_{\{|k| \geqslant N\}}\left(U^{a, j}(k)\left(n_{j}-k_{j}\right) U^{b}(n-k) \mid e^{c}(n)\right) e^{c}(n) .\right)
$$

The term $R_{\text {osc, },}^{\varepsilon, N}$ is localized on the hi-frequencies, hence in order to prove that its $L^{p}\left(\mathbb{R}_{+}, H^{-1,-1 / 2}\right)$ norm tends to zero as $N \rightarrow \infty$ it is sufficient to apply Lebesgue theorem. In fact

$$
\begin{aligned}
\left\|R_{\mathrm{osc}}^{\varepsilon}(U)\right\|_{H^{-1,-1 / 2}} & \leqslant\left\|\mathcal{F}^{-1}\left(\sum_{k+m=n}\left(U^{a}(k) \otimes U^{b}(m) \mid e^{c}(n)\right)_{\mathbb{Z}^{4}} e^{c}(n)\right)\right\|_{H^{0,1 / 2}} \\
& =\|U \otimes U\|_{H^{0,1 / 2}} \\
& \lesssim\|U\|_{H^{1 / 2, s}}^{2} .
\end{aligned}
$$

Now, since $U$ has null horizontal average, we can apply Lemma 2.4 obtaining that

$$
\left\|R_{\mathrm{osc}}^{\varepsilon}(U)\right\|_{H^{-1,-1 / 2}} \lesssim\|U\|_{H^{0, s}}\left\|\nabla_{h} U\right\|_{H^{0, s}} .
$$

Since $L^{2}([0, T]) \subset L^{p^{\prime}}([0, T])$ for $p^{\prime} \in[1,2)$ if we prove that $\left\|R_{\mathrm{osc}}^{\varepsilon}(U)\right\|_{L^{2}\left([0, T] ; H^{-1,-1 / 2}\right)}<\infty$ we can apply Lebesgue theorem and conclude that $\left\|R_{\mathrm{osc}, 1}^{\varepsilon, N}\right\|_{L^{p^{\prime}\left([0, T] ; H^{-1,-1 / 2}\right)}} \rightarrow 0$ as $N \rightarrow \infty$. This is in fact true since

$$
\|\| U\left\|_{H^{0, s}}\right\| \nabla_{h} U\left\|_{H^{0, s}}\right\|_{L_{t}^{2}}^{2}=\int_{0}^{t}\|U(\tau)\|_{H^{0, s}}^{2}\left\|\nabla_{h} U(\tau)\right\|_{H^{0, s}}^{2} \mathrm{~d} \tau \leqslant\|U\|_{L^{\infty}\left(\mathbb{R}_{+} ; H^{0, s}\right)}^{2}\left\|\nabla_{h} U\right\|_{L^{2}\left(\mathbb{R}_{+} ; H^{0, s}\right)}^{2} .
$$

For the second term we argue as follows

$$
\begin{aligned}
\left\|R_{\text {osc }, 2}^{\varepsilon, N}\right\|_{H^{-1,-1 / 2}} & \leqslant\left\|\mathcal{F}^{-1}\left(\sum_{k+m=n} 1_{\{|k| \geqslant N\}}\left(\left(U^{a}(k) \otimes U^{b}(m)\right) \mid e^{c}(n)\right) e^{c}(n)\right)\right\|_{H^{0,1 / 2}} \\
& \leqslant\left\|\mathcal{F}^{-1}\left(\sum_{k+m=n} 1_{\{|k| \geqslant N\}}\left(\hat{U}(k) \mid e^{a}(k)\right) e^{a}(k)\right)\right\|_{H^{1 / 2, s}}\|u\|_{H^{1 / 2, s}},
\end{aligned}
$$

and, using 2.10 we obtain the following bound

$$
\left\|R_{\text {osc }, 2}^{\varepsilon, N}\right\|_{H^{-1,-1 / 2}} \leqslant\left\|\mathcal{F}^{-1}\left(1_{\{|k| \geqslant N\}} U^{a}(k)\right)\right\|_{H^{0, s}}^{1 / 2}\left\|\mathcal{F}^{-1}\left(1_{\{|k| \geqslant N\}}\left(\nabla_{h} U\right)^{a}(k)\right)\right\|_{H^{0, s}}^{1 / 2}\|U\|_{H^{0, s}}^{1 / 2}\left\|\nabla_{h} U\right\|_{H^{0, s}}^{1 / 2},
$$

which evidently tends to zero thanks to Lebesgue theorem.

For the term $S_{\mathrm{osc}, \mathrm{HF}}^{\varepsilon, N}$ it is straightforward since

$$
\left\|S_{\mathrm{osc}, \mathrm{HF}}^{\varepsilon, N}\right\|_{H^{-1, s}}=\left\|\mathcal{F}^{-1}\left(1_{\{|n| \geqslant N\}} \sum_{\omega_{n}^{a, b} \neq 0} e^{i \frac{t}{\varepsilon} \omega_{n}^{a, b}}\left(\mathbf{D}(n) U^{b}(n) \mid e^{a}(n)\right) e^{a}(n)\right)\right\|_{H^{-1, s}} \leqslant C\left\|\nabla_{h} U\right\|_{H^{0, s}} .
$$

\section{The energy estimates}

In this Section we refer to $V_{\mathrm{QG}}$ and $U_{\mathrm{osc}}$ respectively as the solutions of equation [5.11] and [5.10). Moreover $v_{\mathrm{QG}}, u_{\mathrm{osc}}$ represent the projections of the first three components of $V_{\mathrm{QG}}$ and $U_{\mathrm{osc}}$.

The aim of this section is essentially to give an energy bound for the bilinear term appearing in equation (5.10).

Given a generic vector field $u$ we refer to $\underline{u}$ as the horizontal average of $u$. This gives the natural decomposition $u=\underline{u}+\tilde{u}$. Since $\tilde{u}$ has zero horizontal average the results given in the Subsection 2.4 can be applied. 


\subsection{Estimates for the global well-posedness of the limit system}

Proposition 8.1. Let $V_{\mathrm{QG}}=\left(\begin{array}{c}\nabla_{h}^{\perp} \\ 0 \\ -F \partial_{3}\end{array}\right) \Delta_{F}^{-1} \Omega$ where $\Omega$ is the potential vorticity defined in [3.10], then

$$
\begin{aligned}
\left(\Delta_{q}^{\mathrm{v}}\left(v_{\mathrm{QG}}^{h} \cdot \nabla_{h} \Omega\right) \mid \triangle_{q}^{\mathrm{v}} \Omega\right) \leqslant & C 2^{-2 q s} b_{q}(t) \\
& \times\left[\|\Omega\|_{L^{2}\left(\mathbb{T}^{3}\right)}^{1 / 2}\left\|\nabla_{h} \Omega\right\|_{L^{2}\left(\mathbb{T}^{3}\right)}^{1 / 2}\|\Omega\|_{H^{0, s}}^{1 / 2}\left\|\nabla_{h} \Omega\right\|_{H^{0, s}}^{3 / 2}+\left\|\nabla_{h} \Omega\right\|_{L^{2}\left(\mathbb{T}^{3}\right)}\|\Omega\|_{H^{0, s}}\left\|\nabla_{h} \Omega\right\|_{H^{0, s}}\right],
\end{aligned}
$$

where $\left(b_{q}\right)_{q}$ is a $\ell^{1}(\mathbb{Z})$ positive sequence which depends on $\Omega$ and such that $\sum_{q} b_{q}(t) \leqslant 1$.

Proof. Thanks to Bony decomposition 2.3 we can write

$$
\begin{aligned}
& \Delta_{q}^{\mathrm{v}}\left(v_{\mathrm{QG}}^{h} \cdot \nabla_{h} \Omega\right)=S_{q-1}^{v} v_{\mathrm{QG}}^{h} \Delta_{q}^{\mathrm{v}} \nabla_{h} \Omega+ \\
& \quad \sum_{\left|q-q^{\prime}\right| \leqslant 4}\left(\left[\Delta_{q}^{\mathrm{v}} ; S_{q^{\prime}-1}^{\mathrm{v}} v_{\mathrm{QG}}^{h}\right] \Delta_{q^{\prime}}^{\mathrm{v}} \nabla_{h} \Omega+\left(S_{q^{\prime}-1}^{\mathrm{v}} v_{\mathrm{QG}}^{h}-S_{q-1}^{v} v_{\mathrm{QG}}^{h}\right) \triangle_{q}^{\mathrm{v}} \triangle_{q^{\prime}}^{\mathrm{v}} \nabla_{h} \Omega\right)+\sum_{q^{\prime}>q-4} \Delta_{q}^{\mathrm{v}}\left(S_{q^{\prime}+2}^{v} \nabla_{h} \Omega \triangle_{q^{\prime}}^{\mathrm{v}} v_{\mathrm{QG}}^{h}\right),
\end{aligned}
$$

and hence we can decompose $\left(\triangle_{q}^{\mathrm{v}}\left(v_{\mathrm{QG}}^{h} \cdot \nabla_{h} \Omega\right) \mid \triangle_{q}^{\mathrm{v}} \Omega\right)=\sum_{k=1}^{4} I_{h}^{k}(q)$.

First of all, since $\operatorname{div}{ }_{h} v_{\mathrm{QG}}^{h}=0$, we deduce that $I_{h}^{1}=0$. We remark that we proved in Lemma 5.6 that $v_{\mathrm{QG}}^{h}=0$, whence $v_{\mathrm{QG}}^{h}=\tilde{v}_{\mathrm{QG}}^{h}$. Moreover $\nabla_{h} \Omega=\nabla_{h} \tilde{\Omega}$, hence

$$
\begin{aligned}
I_{h}^{2}(q)=\sum_{\left|q-q^{\prime}\right| \leqslant 4} & \left(\left[\Delta_{q}^{\mathrm{v}} ; S_{q^{\prime}-1}^{\mathrm{v}} v_{\mathrm{QG}}^{h}\right] \Delta_{q^{\prime}}^{\mathrm{v}} \nabla_{h} \Omega \mid \triangle_{q}^{\mathrm{v}} \Omega\right) \\
& =\sum_{\left|q-q^{\prime}\right| \leqslant 4}\left(\left[\Delta_{q}^{\mathrm{v}} ; S_{q^{\prime}-1}^{\mathrm{V}} \tilde{v}_{\mathrm{QG}}^{h}\right] \triangle_{q^{\prime}}^{\mathrm{v}} \nabla_{h} \tilde{\Omega} \mid \triangle_{q}^{\mathrm{v}} \tilde{\Omega}\right)+\left(\left[\Delta_{q}^{\mathrm{v}} ; S_{q^{\prime}-1}^{\mathrm{v}} \tilde{v}_{\mathrm{QG}}^{h}\right] \triangle_{q^{\prime}}^{\mathrm{v}} \nabla_{h} \tilde{\Omega} \mid \Delta_{q}^{\mathrm{v}} \Omega\right)=I_{h}^{2,1}(q)+I_{h}^{2,2}(q) .
\end{aligned}
$$

We consider first the term $I_{h}^{2,1}$. By Hölder inequality and Lemma 2.6 we can deduce

$$
I_{h}^{2,1}(q) \lesssim \sum_{\left|q-q^{\prime}\right| \leqslant 4} 2^{-q}\left\|S_{q^{\prime}-1}^{\mathrm{V}} \partial_{3} \tilde{v}_{\mathrm{QG}}^{h}\right\|_{L_{\nu}^{\infty} L_{h}^{4}}\left\|\Delta_{q^{\prime}}^{\mathrm{v}} \nabla_{h} \tilde{\Omega}\right\|_{L^{2}\left(\mathbb{T}^{3}\right)}\left\|\Delta_{q}^{\mathrm{V}} \tilde{\Omega}\right\|_{L_{\nu}^{2} L_{h}^{4}}
$$

we can hence apply [2.9) to the term $\left\|S_{q^{\prime}-1}^{\mathrm{v}} \partial_{3} \tilde{v}_{\mathrm{QG}}^{h}\right\|_{L_{v}^{\infty} L_{h}^{4}}$ and 2.7) to $\left\|\triangle_{q}^{\mathrm{v}} \tilde{\Omega}\right\|_{L_{v}^{2} L_{h}^{4}}$, and then 2.4] and Lemma 5.5 in order to deduce

$$
\begin{aligned}
I_{h}^{2,1}(q) \lesssim & \sum_{\left|q-q^{\prime}\right| \leqslant 4} 2^{-q+q^{\prime} / 2}\left\|\partial_{3} \tilde{v}_{\mathrm{QG}}^{h}\right\|_{L^{2}\left(\mathbb{T}^{3}\right)}^{1 / 2}\left\|\partial_{3} \nabla_{h} \tilde{v}^{h}\right\|_{L^{2}\left(\mathbb{T}^{3}\right)}^{1 / 2}\left\|\Delta_{q^{\prime}}^{\mathrm{v}} \nabla_{h} \tilde{\Omega}\right\|_{L^{2}\left(\mathbb{T}^{3}\right)^{\prime}}, \\
& \times\left\|\triangle_{q}^{\mathrm{v}} \tilde{\Omega}\right\|_{L^{2}\left(\mathbb{T}^{3}\right)}^{1 / 2}\left\|\Delta_{q}^{\mathrm{v}} \nabla_{h} \tilde{\Omega}\right\|_{\left.L^{2} \mathbb{T}^{3}\right)^{\prime}}^{\prime / 2} \\
& \lesssim b_{q}(t) 2^{-q / 2-2 q s}\|\Omega\|_{L^{2}\left(\mathbb{T}^{3}\right)}^{1 / 2}\left\|\nabla_{h} \Omega\right\|_{L^{2}\left(\mathbb{T}^{3}\right)}^{1 / 2}\|\Omega\|_{H^{0, s}}^{1 / 2}\left\|\nabla_{h} \Omega\right\|_{H^{0, s}}^{3 / 2} .
\end{aligned}
$$

For the following terms the tools used are the same as for the term $I_{h}^{2,1}(q)$, hence, we do not explain the procedure in details. For the term $I_{h}^{2,2}(q)$

$$
\begin{aligned}
I_{h}^{2,2}(q) & \lesssim 2^{-q}\left\|S_{q^{\prime}-1}^{\mathrm{v}} \partial_{3} \tilde{v}_{\mathrm{QG}}^{h}\right\|_{L_{v}^{\infty} L_{h}^{2}}\left\|\Delta_{q^{\prime}}^{\mathrm{v}} \nabla_{h} \tilde{\Omega}\right\|_{L^{2}\left(\mathbb{T}^{3}\right)}\left\|\Delta_{q}^{\mathrm{v}} \Omega\right\|_{L_{v}^{2}}, \\
& \lesssim b_{q}(t) 2^{-2 q s-q^{\prime} / 2}\left\|\nabla_{h} \partial_{3} V_{\mathrm{QG}}\right\|_{L^{2}\left(\mathbb{T}^{3}\right)}\|\Omega\|_{H^{0, s}}\left\|\nabla_{h} \Omega\right\|_{H^{0, s}}, \\
& \lesssim b_{q}(t) 2^{-2 q s-q^{\prime} / 2}\left\|\nabla_{h} \Omega\right\|_{L^{2}\left(\mathbb{T}^{3}\right)}\|\Omega\|_{H^{0, s}}\left\|\nabla_{h} \Omega\right\|_{H^{0, s}},
\end{aligned}
$$

where in the first inequality we used (2.8) and by Poincaré inequality in the horizontal variable to obtain

$$
\left\|S_{q^{\prime}-1}^{\mathrm{v}} \partial_{3} \tilde{v}_{\mathrm{QG}}^{h}\right\|_{L_{v}^{\infty} L_{h}^{2}} \lesssim 2^{q^{\prime} / 2}\left\|S_{q^{\prime}-1}^{\mathrm{v}} \nabla_{h} \partial_{3} \tilde{v}_{\mathrm{QG}}^{h}\right\|_{L^{2}\left(\mathbb{T}^{3}\right)}
$$

Next, we consider the term

$$
\begin{aligned}
& I_{h}^{3}(q)=\sum_{\left|q-q^{\prime}\right| \leqslant 4}\left(\left(S_{q^{\prime}-1}^{\mathrm{v}} v_{\mathrm{QG}}^{h}-S_{q-1}^{\nu} v_{\mathrm{QG}}^{h}\right) \triangle_{q}^{\mathrm{v}} \triangle_{q^{\prime}}^{\mathrm{v}} \nabla_{h} \Omega \mid \triangle_{q}^{\mathrm{v}} \Omega\right) \\
& =\sum_{\left|q-q^{\prime}\right| \leqslant 4}\left(\left(S_{q^{\prime}-1}^{\mathrm{v}} \tilde{v}_{\mathrm{QG}}^{h}-S_{q-1}^{v} \tilde{v}_{\mathrm{QG}}^{h}\right) \triangle_{q}^{\mathrm{v}} \triangle_{q^{\prime}}^{\mathrm{v}} \nabla_{h} \tilde{\Omega} \mid \triangle_{q^{\mathrm{V}}}^{\mathrm{v}} \tilde{\Omega}\right) \\
& +\left(\left(S_{q^{\prime}-1}^{\mathrm{v}} \tilde{\mathrm{Q}}_{\mathrm{QG}}^{h}-S_{q-1}^{v} \tilde{v}_{\mathrm{QG}}^{h}\right) \triangle_{q}^{\mathrm{v}} \triangle_{q^{\prime}}^{\mathrm{v}} \nabla_{h} \tilde{\Omega} \mid \triangle_{q}^{\mathrm{v}} \Omega\right)=I_{h}^{3,1}(q)+I_{h}^{3,2}(q) .
\end{aligned}
$$


With calculations similar and since Supp $\mathcal{F}\left(S_{q^{\prime}-1}^{\mathrm{v}} v_{\mathrm{QG}}^{h}-S_{q-1}^{v} v_{\mathrm{QG}}^{h}\right) \subset \bigcup_{\left|q-q^{\prime}\right| \leqslant 4} 2^{q} \mathcal{C}$, and hence localized from above and below in the frequency space, using respectively in the first inequality [2.9], Bernstein inequality, 2.7], 2.4 and Lemma 5.5

$$
\begin{aligned}
I_{h}^{3,1}(q) & \leqslant \sum_{\left|q-q^{\prime}\right| \leqslant 4}\left\|S_{q^{\prime}-1}^{\mathrm{v}} \tilde{v}_{\mathrm{QG}}^{h}-S_{q-1}^{v} \tilde{v}_{\mathrm{QG}}^{h}\right\|\left\|_{L_{v}^{\infty} L_{h}^{4}}\right\| \Delta_{q}^{\mathrm{v}} \Delta_{q^{\prime}}^{\mathrm{v}} \nabla_{h} \tilde{\Omega}\left\|_{L^{2}\left(\mathbb{T}^{3}\right)}\right\| \Delta_{q}^{\mathrm{v}} \tilde{\Omega} \|_{L_{v}^{2} L_{h}^{4}}, \\
& \lesssim b_{q}(t) 2^{-q / 2-2 q s}\left\|\partial_{3} V_{\mathrm{QG}}\right\|_{L^{2}\left(\mathbb{T}^{3}\right)}^{1 / 2}\left\|\nabla_{h} \partial_{3} V_{\mathrm{QG}}\right\|_{L^{2}\left(\mathbb{T}^{3}\right)}^{1 / 2}\|\Omega\|_{H^{0, s}}^{1 / 2}\left\|\nabla_{h} \Omega\right\|_{H^{0, s}}^{3 / 2}, \\
& \lesssim b_{q}(t) 2^{-q / 2-2 q s}\|\Omega\|_{L^{2}\left(\mathbb{T}^{3}\right)}^{1 / 2}\left\|\nabla_{h} \Omega\right\|_{L^{2}\left(\mathbb{T}^{3}\right)}^{1 / 2}\|\Omega\|_{H^{0, s}}^{1 / 2}\left\|\nabla_{h} \Omega\right\|_{H^{0, s}}^{3 / 2} .
\end{aligned}
$$

The procedure for the term $I_{h}^{3,2}(q)$ is almost the same as the one for the term $I_{h}^{3,1}(q)$, except that we do not use 2.7 and we use Poincaré inequality in the horizontal variables

$$
I_{h}^{3,2}(q) \leqslant b_{q}(t) 2^{-q / 2-2 q s}\left\|\nabla_{h} \Omega\right\|_{L^{2}\left(\mathbb{T}^{3}\right)}\|\Omega\|_{H^{0, s}}\left\|\nabla_{h} \Omega\right\|_{H^{0, s}} .
$$

The last term

$$
\begin{aligned}
I_{h}^{4}(q)=\sum_{q^{\prime}>q-1} & \left(\Delta_{q}^{\mathrm{v}}\left(S_{q^{\prime}+2}^{v} \nabla_{h} \Omega \Delta_{q^{\prime}}^{\mathrm{v}} v_{\mathrm{QG}}^{h}\right) \mid \Delta_{q}^{\mathrm{v}} \Omega\right) \\
& =\sum_{q^{\prime}>q-1}\left(\Delta_{q}^{\mathrm{v}}\left(S_{q^{\prime}+2}^{v} \nabla_{h} \Omega \Delta_{q^{\prime}}^{\mathrm{v}} v_{\mathrm{QG}}^{h}\right) \mid \Delta_{q}^{\mathrm{v}} \tilde{\Omega}\right)+\sum_{q^{\prime}>q-1}\left(\Delta_{q}^{\mathrm{v}}\left(S_{q^{\prime}+2}^{v} \nabla_{h} \Omega \Delta_{q^{\prime}}^{\mathrm{v}} v_{\mathrm{QG}}^{h}\right) \mid \Delta_{q}^{\mathrm{v}} \Omega\right)=I_{h}^{4,1}(q)+I_{h}^{4,2}(q) .
\end{aligned}
$$

Let us deal with the term $I_{h}^{4,1}(q)$. Applying Hölder inequality we deduce

$$
I_{h}^{4,1}(q) \leqslant \sum_{q^{\prime}>q-1}\left\|\triangle_{q^{\prime}}^{\mathrm{v}} v_{\mathrm{QG}}^{h}\right\|_{L_{v}^{\infty} L_{h}^{4}}\left\|S_{q^{\prime}+2}^{v} \nabla_{h} \Omega\right\|_{L^{2}\left(\mathbb{T}^{3}\right)}\left\|\triangle_{q}^{\mathrm{v}} \tilde{\Omega}\right\|_{L_{v}^{2} L_{h}^{4}} .
$$

Using Bernstein inequality twice, 2.7], Lemma 5.5 and lastly 2.4 we deduce

$$
\begin{aligned}
\left\|\triangle_{q^{\prime}}^{\mathrm{v}} v_{\mathrm{QG}}^{h}\right\|_{L_{v}^{\infty} L_{h}^{4}} & \lesssim 2^{q^{\prime} / 2}\left\|\triangle_{q^{\prime}}^{\mathrm{v}} v_{\mathrm{QG}}^{h}\right\|_{L_{v}^{2} L_{h}^{4}}, \\
& \lesssim 2^{-q^{\prime} / 2}\left\|\partial_{3} \triangle_{q^{\prime}}^{\mathrm{v}} v_{\mathrm{QG}}^{h}\right\|_{L_{v}^{2} L_{h}^{4}}, \\
& \lesssim 2^{-q^{\prime} / 2}\left\|\partial_{3} \triangle_{q^{\prime}}^{\mathrm{v}} v_{\mathrm{QG}}^{h}\right\|_{L^{2}\left(\mathbb{T}^{3}\right)}^{1 / 2}\left\|\partial_{3} \nabla_{h} \triangle_{q^{\prime}}^{\mathrm{v}} v_{\mathrm{QG}}^{h}\right\|_{L^{2}\left(\mathbb{T}^{3}\right)}^{1 / 2}, \\
& \lesssim 2^{-q^{\prime} / 2}\left\|\triangle_{q^{\prime}}^{\mathrm{v}} \Omega\right\|_{L^{2}\left(\mathbb{T}^{3}\right)}^{1 / 2}\left\|\nabla_{h} \triangle_{q^{\prime}}^{\mathrm{v}} \Omega\right\|_{L^{2}\left(\mathbb{J}^{3}\right)}^{1 / 2}, \\
& \lesssim c_{q^{\prime}}(\Omega, t) 2^{-q^{\prime} / 2-q^{\prime} s}\|\Omega\|_{H^{0, s}}^{1 / 2}\left\|\nabla_{h} \Omega\right\|_{H^{0, s}}^{1 / 2} .
\end{aligned}
$$

An application of 2.7] and 2.4 gives instead

$$
\left\|\triangle_{q}^{\mathrm{v}} \tilde{\Omega}\right\|_{L_{v}^{2} L_{h}^{4}} \lesssim c_{q}(\Omega, t) 2^{-q s}\|\Omega\|_{H^{0, s}}^{1 / 2}\left\|\nabla_{h} \Omega\right\|_{H^{0, s}}^{1 / 2},
$$

whence we deduce the bound

$$
I_{h}^{4,1}(q) \leqslant C 2^{-2 q s-q / 2} b_{q}(\Omega, t)\left\|\nabla_{h} \Omega\right\|_{L^{2}\left(\mathbb{T}^{3}\right)}\|\Omega\|_{H^{0, s}}\left\|\nabla_{h} \Omega\right\|_{H^{0, s}} .
$$

To bound the term $I_{h}^{4,2}(q)$ is a similar procedure and hence is omitted. Collecting the estimates 8.3 -8.7) we deduce the bound [8.1].

Proposition 8.2. Let $V_{\mathrm{QG}}$ and $U_{\mathrm{O} \text { c }}$ respectively be the solution of equation [5.11] and (5.10), then if the horizontal mean of $V_{\mathrm{QG}}$ and $U_{\mathrm{Osc}}$ is zero (see Lemmas 5.6 and 5.7) the following estimates hold

$$
\begin{aligned}
& \left(\triangle_{q}^{\mathrm{v}} \mathcal{Q}\left(V_{\mathrm{QG}}, U_{\mathrm{osc}}\right) \mid \triangle_{q}^{\mathrm{v}} U_{\mathrm{osc}}\right)_{L^{2}\left(\mathbb{T}^{3}\right)}+\left(\triangle_{q}^{\mathrm{v}} \mathcal{Q}\left(U_{\mathrm{osc}}, V_{\mathrm{QG}}\right) \mid \triangle_{q}^{\mathrm{v}} U_{\mathrm{osc}}\right)_{L^{2}\left(\mathbb{T}^{3}\right)} \\
& \leqslant C 2^{-2 q s} b_{q}(t)\left\|\nabla_{h} \Omega\right\|_{H^{0, s}}\left\|\nabla_{h} U_{\mathrm{osc}}\right\|_{H^{0, s}}\left\|U_{\mathrm{osc}}\right\|_{H^{0, s}} \\
& +C 2^{-2 q s} b_{q}(t)\|\Omega\|_{H^{0, s}}^{1 / 2}\left\|\nabla_{h} \Omega\right\|_{H^{0, s}}^{1 / 2}\left\|U_{\mathrm{osc}}\right\|_{H^{0, s}}^{1 / 2}\left\|\nabla_{h} U_{\mathrm{osc}}\right\|_{H^{0, s}}^{3 / 2} \\
& \left(\triangle_{q}^{\mathrm{v}} \mathcal{Q}\left(U_{\mathrm{osc}}, U_{\mathrm{osc}}\right) \mid \triangle_{q}^{\mathrm{v}} U_{\mathrm{osc}}\right)_{L^{2}\left(\mathbb{T}^{3}\right)} \leqslant C b_{q}(t) 2^{-2 q s}\left\|\nabla_{h} U_{\mathrm{osc}}\right\|_{L^{2}\left(\mathbb{T}^{3}\right)}\left\|U_{\mathrm{osc}}\right\|_{H^{0, s}}\left\|\nabla_{h} U_{\mathrm{osc}}\right\|_{H^{0, s}} \\
& +C b_{q}(t) 2^{-2 q s}\left\|U_{\mathrm{osc}}\right\|_{L^{2}\left(\mathbb{T}^{3}\right)}^{1 / 2}\left\|\nabla_{h} U_{\mathrm{osc}}\right\|_{L^{2}\left(\mathbb{T}^{3}\right)}^{1 / 2}\left\|U_{\mathrm{osc}}\right\|_{H^{0, s}}^{1 / 2}\left\|\nabla_{h} U_{\mathrm{osc}}\right\|_{H^{0, s}}^{3 / 2} .
\end{aligned}
$$

The sequence $\left(b_{q}\right)_{q}$ is a $\ell^{1}(\mathbb{Z})$ positive sequence which depends on $\Omega, U_{\mathrm{osc}}$ and such that $\sum_{q} b_{q}(t) \leqslant 1$. 
Remark 8.3. From now on $(\cdot \mid \cdot)=(\cdot \mid \cdot)_{L^{2}\left(\mathbb{T}^{3}\right)}$

Proof. We divide the proof of the above proposition in two parts, namely one part for each estimate.

In what follows we always consider $s>1 / 2$, hence the embedding $H_{v}^{s} \hookrightarrow L_{v}^{\infty}$ holds true. Moreover we underline the fact that $V_{\mathrm{QG}}(t)$ and $U_{\mathrm{osc}}(t)$ have zero horizontal average for each $t>0$ if the initial data has zero horizontal average thanks to the results of Lemma 5.6 and Lemma 5.7. whence the estimates [2.7), 2.10) and Lemma 1.2 can be applied in this context.

Proof of 8.8: in order to prove the estimate 8.8 we substitute the bilinear form $\mathcal{Q}$ with the transport bilinear form. This choice is done only in order to simplify the notation.

Indeed we have

$$
\begin{aligned}
\left|\left(\triangle_{q}^{\mathrm{v}}\left(v_{\mathrm{QG}} \cdot \nabla U_{\mathrm{osc}}\right) \mid \triangle_{q}^{\mathrm{v}} U_{\mathrm{osc}}\right)\right|= & \left|\left(\triangle_{q}^{\mathrm{v}}\left(v_{\mathrm{QG}}^{h} \cdot \nabla_{h} U_{\mathrm{osc}}\right) \mid \triangle_{q}^{\mathrm{v}} U_{\mathrm{osc}}\right)\right|, \\
= & \left|\left(\operatorname{div}_{h} \triangle_{q}^{\mathrm{v}}\left(v_{\mathrm{QG}}^{h} \otimes U_{\mathrm{osc}}\right) \mid \triangle_{q}^{\mathrm{v}} U_{\mathrm{osc}}\right)\right|, \\
\left|\left(\triangle_{q}^{\mathrm{v}}\left(u_{\mathrm{osc}} \cdot \nabla V_{\mathrm{QG}}\right) \mid \triangle_{q}^{\mathrm{v}} U_{\mathrm{osc}}\right)\right| \leqslant & \left|\left(\operatorname{div}_{h} \triangle_{q}^{\mathrm{v}}\left(u_{\mathrm{osc}}^{h} \otimes V_{\mathrm{QG}}\right) \mid \triangle_{q}^{\mathrm{v}} U_{\mathrm{osc}}\right)\right| \\
& +\left|\left(\partial_{3} \triangle_{q}^{\mathrm{v}}\left(u_{\mathrm{osc}}^{3} V_{\mathrm{QG}}\right) \mid \triangle_{q}^{\mathrm{v}} U_{\mathrm{osc}}\right)\right|,
\end{aligned}
$$

and indeed

$$
\left|\left(\operatorname{div}_{h} \triangle_{q}^{\mathrm{v}}\left(v_{\mathrm{QG}}^{h} \otimes U_{\mathrm{osc}}\right) \mid \triangle_{q}^{\mathrm{v}} U_{\mathrm{osc}}\right)\right|+\left|\left(\operatorname{div}_{h} \triangle_{q}^{\mathrm{v}}\left(u_{\mathrm{osc}}^{h} \otimes V_{\mathrm{QG}}\right) \mid \triangle_{q}^{\mathrm{v}} U_{\mathrm{osc}}\right)\right| \leqslant 2\left|\left(\triangle_{q}^{\mathrm{v}}\left(U_{\mathrm{osc}} \otimes V_{\mathrm{QG}}\right) \mid \triangle_{q}^{\mathrm{v}} \nabla_{h} U_{\mathrm{osc}}\right)\right|,
$$

whence

$$
\begin{aligned}
\left|\left(\triangle_{q}^{\mathrm{v}}\left(v_{\mathrm{QG}} \cdot \nabla U_{\mathrm{osc}}\right) \mid \triangle_{q}^{\mathrm{v}} U_{\mathrm{osc}}\right)\right| & +\left|\left(\triangle_{q}^{\mathrm{v}}\left(u_{\mathrm{osc}} \cdot \nabla V_{\mathrm{QG}}\right) \mid \triangle_{q}^{\mathrm{v}} U_{\mathrm{osc}}\right)\right| \\
& \leqslant 2\left|\left(\triangle_{q}^{\mathrm{v}}\left(U_{\mathrm{osc}} \otimes V_{\mathrm{QG}}\right) \mid \triangle_{q}^{\mathrm{v}} \nabla_{h} U_{\mathrm{osc}}\right)\right|+\left|\left(\partial_{3} \triangle_{q}^{\mathrm{v}}\left(u_{\mathrm{osc}}^{3} V_{\mathrm{QG}}\right) \mid \triangle_{q}^{\mathrm{v}} U_{\mathrm{osc}}\right)\right|=B_{h}(q)+B_{v}(q) .
\end{aligned}
$$

Thanks to 2.4 and Lemma 1.2 we deduce

$$
\begin{aligned}
B_{h}(q) & \lesssim 2^{-2 q s} b_{q}(t)\left\|U_{\mathrm{osc}} \otimes V_{\mathrm{QG}}\right\|_{H^{0, s}}\left\|\nabla_{h} U_{\mathrm{osc}}\right\|_{H^{0, s}} \\
& \lesssim 2^{-2 q s} b_{q}(t)\left\|V_{\mathrm{QG}}\right\|_{H^{1 / 2, s}}\left\|U_{\mathrm{osc}}\right\|_{H^{1 / 2, s}}\left\|\nabla_{h} U_{\mathrm{osc}}\right\|_{H^{0, s}}
\end{aligned}
$$

An application of Poincaré inequality and and 2.10 allows us to deduce that

$$
\begin{aligned}
\left\|V_{\mathrm{QG}}\right\|_{H^{1 / 2, s}} & \lesssim\left\|\nabla_{h} V_{\mathrm{QG}}\right\|_{H^{1 / 2, s}}, \\
& \lesssim\left\|\nabla_{h} V_{\mathrm{QG}}\right\|_{H^{0, s}}^{1 / 2}\left\|\nabla_{h}^{2} V_{\mathrm{QG}}\right\|_{H^{0, s}}^{1 / 2} .
\end{aligned}
$$

An application of Lemma 5.5 leads to

$$
\left\|\nabla_{h} V_{\mathrm{QG}}\right\|_{H^{0, s}}^{1 / 2}\left\|\nabla_{h}^{2} V_{\mathrm{QG}}\right\|_{H^{0, s}}^{1 / 2} \lesssim\|\Omega\|_{H^{0, s}}^{1 / 2}\left\|\nabla_{h} \Omega\right\|_{H^{0, s}}^{1 / 2},
$$

whence with use of 2.10 we deduce the bound

$$
B_{h}(q) \lesssim 2^{-2 q s} b_{q}(t)\|\Omega\|_{H^{0, s}}^{1 / 2}\left\|\nabla_{h} \Omega\right\|_{H^{0, s}}^{1 / 2}\left\|U_{\mathrm{osc}}\right\|_{H^{0, s}}^{1 / 2}\left\|\nabla_{h} U_{\mathrm{osc}}\right\|_{H^{0, s}}^{3 / 2} .
$$

The term $B_{\nu}$ can instead be written as

$$
B_{v}(q)=\left|\left(\triangle_{q}^{\mathrm{v}}\left(\operatorname{div}_{h} u_{\mathrm{osc}}^{h} V_{\mathrm{QG}}\right) \mid \triangle_{q}^{\mathrm{v}} U_{\mathrm{osc}}\right)\right|+\left|\left(\triangle_{q}^{\mathrm{v}}\left(u_{\mathrm{osc}}^{3} \partial_{3} V_{\mathrm{QG}}\right) \mid \triangle_{q}^{\mathrm{v}} U_{\mathrm{osc}}\right)\right|=B_{v}^{1}(q)+B_{v}^{2}(q) .
$$

For the term $B_{v}^{1}(q)$, applying 2.4 and Lemma 1.2

$$
\begin{aligned}
B_{v}^{1}(q) & \lesssim 2^{-2 q s} b_{q}(t)\left(\Omega, U_{\mathrm{osc}}\right)\left\|\operatorname{div}_{h} u_{\mathrm{osc}}^{h} V_{\mathrm{QG}}\right\|_{H^{-1 / 2, s}}\left\|U_{\mathrm{osc}}\right\|_{H^{1 / 2, s}}, \\
& \lesssim 2^{-2 q s} b_{q}(t)\left\|V_{\mathrm{QG}}\right\|_{H^{1 / 2, s}}\left\|U_{\mathrm{osc}}\right\|_{H^{1 / 2, s}}\left\|\nabla_{h} U_{\mathrm{osc}}\right\|_{H^{0, s}},
\end{aligned}
$$

which is the same estimate as 8.10 and whence we can deduce the same bound as for $B_{h}(q)$. i.e. 8.11].

The term $B_{v}^{2}(q)$ is indeed less regular due to the presence of the vertical derivative. Similarly as before we can apply (2.4) and Lemma 1.2 to deduce

$$
B_{v}^{2}(q) \lesssim 2^{-2 q s} b_{q}(t)\left\|\partial_{3} V_{\mathrm{QG}}\right\|_{H^{0, s}}\left\|U_{\mathrm{OSc}}\right\|_{H^{1 / 2, s}}^{2} .
$$


Poincaré inequality and Lemma 5.5 imply

$$
\left\|\partial_{3} V_{\mathrm{QG}}\right\|_{H^{0, s}} \lesssim\left\|\partial_{3} \nabla_{h} V_{\mathrm{QG}}\right\|_{H^{0, s}} \lesssim\left\|\nabla_{h} \Omega\right\|_{H^{0, s}},
$$

while using 2.10 we can conclude with the following bound

$$
B_{v}^{2}(q) \lesssim 2^{-2 q s} b_{q}(t)\left\|\nabla_{h} \Omega\right\|_{H^{0, s}}\left\|U_{\mathrm{osc}}\right\|_{H^{0, s}}\left\|\nabla_{h} U_{\mathrm{osc}}\right\|_{H^{0, s}} .
$$

Whence 8.11 and 8.12 prove 8.8 .

Proof of [8.9]: Lastly we consider the term

$$
\begin{aligned}
\left(\triangle_{q}^{\mathrm{v}} \mathcal{Q}\left(U_{\mathrm{osc}}, U_{\mathrm{osc}}\right) \mid \triangle_{q}^{\mathrm{v}} U_{\mathrm{osc}}\right) & =\left(\triangle_{q}^{\mathrm{v}} \mathcal{Q}^{h}\left(U_{\mathrm{osc}}, U_{\mathrm{osc}}\right) \mid \triangle_{q}^{\mathrm{v}} U_{\mathrm{osc}}\right)+\left(\triangle_{q}^{\mathrm{v}} \mathcal{Q}^{3}\left(U_{\mathrm{osc}}, U_{\mathrm{osc}}\right) \mid \triangle_{q}^{\mathrm{v}} U_{\mathrm{osc}}\right) \\
& =C^{h}(q)+C^{v}(q),
\end{aligned}
$$

where $\mathcal{Q}^{h}$ and $\mathcal{Q}^{3}$ are respectively defined as

$$
\begin{aligned}
& \mathcal{Q}^{h}\left(U_{\mathrm{osc}}, U_{\mathrm{osc}}\right)=\lim _{\varepsilon \rightarrow 0} \mathcal{L}\left(-\frac{t}{\varepsilon}\right)\left[\left(\mathcal{L}\left(\frac{t}{\varepsilon}\right) U_{\mathrm{osc}}^{\varepsilon}\right)^{h} \cdot \nabla_{h} \mathcal{L}\left(\frac{t}{\varepsilon}\right) U_{\mathrm{osc}}\right], \\
& \mathcal{Q}^{3}\left(U_{\mathrm{osc}}, U_{\mathrm{osc}}\right)=\lim _{\varepsilon \rightarrow 0} \mathcal{L}\left(-\frac{t}{\varepsilon}\right)\left[\left(\mathcal{L}\left(\frac{t}{\varepsilon}\right) U_{\mathrm{osc}}^{\varepsilon}\right)^{3} \partial_{3} \mathcal{L}\left(\frac{t}{\varepsilon}\right) U_{\mathrm{osc}}\right]
\end{aligned}
$$

By aid of Bony decomposition as in 2.2] we can say that

$$
\begin{aligned}
C^{h}(q)=\sum_{\left|q-q^{\prime}\right| \leqslant 4}\left(\triangle_{q}^{\mathrm{v}} \mathcal{Q}^{h}\left(S_{q^{\prime}-1}^{\mathrm{v}} U_{\mathrm{osc}}, \triangle_{q^{\prime}}^{\mathrm{v}} U_{\mathrm{osc}}\right) \mid\right. & \left.\Delta_{q}^{\mathrm{v}} U_{\mathrm{osc}}\right) \\
& +\sum_{q^{\prime}>q-4}\left(\triangle_{q}^{\mathrm{v}} \mathcal{Q}^{h}\left(\triangle_{q^{\prime}}^{\mathrm{v}} U_{\mathrm{osc}}, S_{q^{\prime}+2}^{v} U_{\mathrm{osc}}\right) \mid \triangle_{q}^{\mathrm{v}} U_{\mathrm{osc}}\right)=C_{1}^{h}(q)+C_{2}^{h}(q) .
\end{aligned}
$$

By use of Lemma 8.4

$$
C_{1}^{h}(q) \lesssim \sum_{\left|q-q^{\prime}\right| \leqslant 4}\left\|S_{q^{\prime}-1}^{\mathrm{v}} U_{\mathrm{osc}}\right\|_{H^{1 / 2,0}}\left\|\Delta_{q^{\prime}}^{\mathrm{v}} \nabla_{h} U_{\mathrm{osc}}\right\|_{L^{2}\left(\mathbb{T}^{3}\right)}\left\|\Delta_{q}^{\mathrm{v}} U_{\mathrm{osc}}\right\|_{H^{1 / 2,0}},
$$

moreover since $U_{\text {osc }}$ is a vector field with zero horizontal average we can apply 2.7

$$
\begin{aligned}
\left\|S_{q^{\prime}-1}^{\mathrm{v}} U_{\mathrm{osc}}\right\|_{H^{1 / 2,0}} & \lesssim\left\|U_{\mathrm{osc}}\right\|_{L^{2}\left(\mathbb{T}^{3}\right)}^{1 / 2}\left\|\nabla_{h} U_{\mathrm{osc}}\right\|_{L^{2}\left(\mathbb{T}^{3}\right)}^{1 / 2}, \\
\left\|\triangle_{q}^{\mathrm{v}} U_{\mathrm{osc}}\right\|_{H^{1 / 2,0}} & \lesssim\left\|\triangle_{q}^{\mathrm{v}} U_{\mathrm{osc}}\right\|_{L^{2}\left(\mathbb{T}^{3}\right)}^{1 / 2}\left\|\nabla_{h} \triangle_{q}^{\mathrm{v}} U_{\mathrm{osc}}\right\|_{L^{2}\left(\mathbb{T}^{3}\right)}^{1 / 2},
\end{aligned}
$$

whence thanks to 2.4 and the fact that the sum is performed on a finite set of $q^{\prime}$

$$
C_{1}^{h}(q) \lesssim b_{q}(t) 2^{-2 q s}\left\|U_{\mathrm{osc}}\right\|_{L^{2}\left(\mathbb{T}^{3}\right)}^{1 / 2}\left\|\nabla_{h} U_{\mathrm{osc}}\right\|_{L^{2}\left(\mathbb{T}^{3}\right)}^{1 / 2}\left\|U_{\mathrm{osc}}\right\|_{H^{0, s}}^{1 / 2}\left\|\nabla_{h} U_{\mathrm{osc}}\right\|_{H^{0, s}}^{3 / 2} .
$$

Similar computations give us the result for $C_{2}^{h}$, here we sketch the procedure. Respectively using [8.21, 2.4] and summing on the summation set

$$
\begin{aligned}
C_{2}^{h}(q) & =\sum_{q^{\prime}>q-4}\left(\triangle_{q}^{\mathrm{v}} \mathcal{Q}^{h}\left(\triangle_{q^{\prime}}^{\mathrm{v}} U_{\mathrm{osc}}, S_{q^{\prime}+2}^{\nu} U_{\mathrm{osc}}\right) \mid \triangle_{q}^{\mathrm{v}} U_{\mathrm{osc}}\right) \\
& \lesssim b_{q}(t) 2^{-2 q s}\left\|\nabla_{h} U_{\mathrm{osc}}\right\|_{L^{2}\left(\mathbb{T}^{3}\right)}\left\|U_{\mathrm{osc}}\right\|_{H^{0, s}}\left\|\nabla_{h} U_{\mathrm{osc}}\right\|_{H^{0, s}} .
\end{aligned}
$$

On the term $C^{v}$ we apply instead Bony decomposition as in 8.2 obtaining

$$
\begin{aligned}
& C^{\nu}(q)=\left(\mathcal{Q}^{3}\left(S_{q-1}^{v} U_{\mathrm{osc}}, \Delta_{q}^{\mathrm{v}} U_{\mathrm{osc}}\right) \mid \triangle_{q}^{\mathrm{v}} U_{\mathrm{osc}}\right) \\
&+\sum_{\left|q-q^{\prime}\right| \leqslant 4}\left(\mathcal{Q}^{3}\left(S_{q-1}^{v} U_{\mathrm{osc}}-S_{q^{\prime}-1}^{v} U_{\mathrm{osc}}, \triangle_{q}^{\mathrm{v}} \triangle_{q^{\prime}}^{\mathrm{v}} U_{\mathrm{osc}}\right) \mid \triangle_{q}^{\mathrm{v}} U_{\mathrm{osc}}\right) \\
&+\sum_{\left|q-q^{\prime}\right| \leqslant 4} \lim _{\varepsilon \rightarrow 0}\left(\left[\triangle_{q}^{\mathrm{v}}, S_{q^{\prime}-1}^{v}\left(\mathcal{L}\left(\frac{t}{\varepsilon}\right) U_{\mathrm{osc}}\right)^{3}\right] \triangle_{q^{\prime}}^{\mathrm{v}}, \partial_{3} \mathcal{L}\left(\frac{t}{\varepsilon}\right) U_{\mathrm{osc}} \mid \triangle_{q}^{\mathrm{v}} \mathcal{L}\left(\frac{t}{\varepsilon}\right) U_{\mathrm{osc}}\right) \\
&+\sum_{q^{\prime}>q-4}\left(\triangle_{q}^{\mathrm{v}} \mathcal{Q}^{3}\left(\triangle_{q^{\prime}}^{\mathrm{v}} U_{\mathrm{osc}}, S_{q^{\prime}+2}^{v} U_{\mathrm{osc}}\right) \mid \triangle_{q}^{\mathrm{v}} U_{\mathrm{osc}}\right)=\sum_{k=1}^{4} C_{k}^{v}(q),
\end{aligned}
$$


where $\mathcal{Q}^{3}$ is defined in 8.14 . Let us consider the term $C_{1}^{\nu}(q)$ first. Integration by parts and the fact that divergencefree vector fields are considered gives

$$
\begin{aligned}
C_{1}^{\nu}(q) & =\lim _{\varepsilon \rightarrow 0} \int_{\mathbb{T}^{3}} S_{q-1}^{v}\left(\mathcal{L}\left(\frac{t}{\varepsilon}\right) U_{\mathrm{osc}}\right)^{3} \partial_{3} \mathcal{L}\left(\frac{t}{\varepsilon}\right) \triangle_{q}^{\mathrm{v}} U_{\mathrm{osc}} \mathcal{L}\left(\frac{t}{\varepsilon}\right) \triangle_{q}^{\mathrm{v}} U_{\mathrm{osc}} \mathrm{d} x, \\
& =-\frac{1}{2} \lim _{\varepsilon \rightarrow 0} \int_{\mathbb{T}^{3}} S_{q-1}^{\nu} \operatorname{div}_{h}\left(\mathcal{L}\left(\frac{t}{\varepsilon}\right) U_{\mathrm{osc}}\right)^{h}\left|\mathcal{L}\left(\frac{t}{\varepsilon}\right) \triangle_{q}^{\mathrm{v}} U_{\mathrm{osc}}\right|^{2} \mathrm{~d} x .
\end{aligned}
$$

Using the fact that $\mathcal{L}\left(\frac{t}{\varepsilon}\right)$ is an isometry on Sobolev spaces, and 8.21 we deduce

$$
\begin{aligned}
C_{1}^{\nu}(q) & =\lim _{\varepsilon \rightarrow 0} \int_{\mathbb{T}^{3}} S_{q-1}^{v} \operatorname{div}_{h}\left(\mathcal{L}\left(\frac{t}{\varepsilon}\right) U_{\mathrm{osc}}\right)^{h}\left(\mathcal{L}\left(\frac{t}{\varepsilon}\right) \triangle_{q}^{\mathrm{v}} U_{\mathrm{osc}}\right)^{2} \mathrm{~d} x, \\
& \lesssim b_{q}(t) 2^{-2 q s}\left\|\nabla_{h} U_{\mathrm{osc}}\right\|_{L^{2}\left(\mathbb{T}^{3}\right)}\left\|U_{\mathrm{osc}}\right\|_{H^{0, s}}\left\|\nabla_{h} U_{\mathrm{osc}}\right\|_{H^{0, s}} .
\end{aligned}
$$

Let us consider the term $C_{2}^{\nu}$ which is defined as

$$
\begin{aligned}
C_{2}^{v}(q) & \left.=\sum_{\left|q-q^{\prime}\right| \leqslant 4}\left(\mathcal{Q}^{3}\left(S_{q-1}^{v} U_{\mathrm{osc}}-S_{q^{\prime}-1}^{v} U_{\mathrm{osc}}, \triangle_{q}^{\mathrm{v}} \Delta_{q^{\prime}}^{\mathrm{v}} U_{\mathrm{osc}}\right)\right) \mid \Delta_{q}^{\mathrm{v}} U_{\mathrm{osc}}\right), \\
& =\sum_{\left|q-q^{\prime}\right| \leqslant 4} \sum_{\substack{(k, m, n) \in \mathcal{K}^{\star} \\
a, b, c, d= \pm}}\left(\widehat{S_{q-1}^{v} U}{ }^{a, 3}(k)-{\widehat{S_{q^{\prime}-1}^{v} U}}^{a, 3}(k)\right) m_{3}{\widehat{\Delta_{q}^{\mathrm{v}} \triangle_{q^{\prime}}^{\mathrm{v}} U}}^{b, c}(m, n){\widehat{\triangle_{q}^{\mathrm{v}} U}}^{d}(n),
\end{aligned}
$$

where $\hat{U}^{b, c}(m, n)=\left(\hat{U}^{b}(m) \mid e^{c}(n)\right) e^{c}(n)$. Since the eigenvectors $e^{c}$ are normalized to norm one we deduce $\left|\hat{U}^{b, c}(m, n)\right| \lesssim$ $\left|U^{b}(m)\right|$. At this point we can use Lemma 8.4 to obtain the bound

$$
C_{2}^{\nu}(q) \lesssim \sum_{\left|q-q^{\prime}\right| \leqslant 4} \sum_{a= \pm}\left\|S_{q-1}^{v} U^{a, 3}-S_{q^{\prime}-1}^{v} U^{a, 3}\right\|_{L^{2}\left(\mathbb{T}^{3}\right)}\left\|\triangle_{q}^{\mathrm{v}} \triangle_{q^{\prime}}^{\mathrm{v}} \partial_{3} U_{\mathrm{osc}}\right\|_{H^{1 / 2,0}}\left\|\triangle_{q}^{\mathrm{v}} U_{\mathrm{osc}}\right\|_{H^{1 / 2,0}} .
$$

We remark that the term $U^{a}$ is in fact divergence-free.

Thanks to Lemma2.1

$$
\begin{aligned}
\left\|S_{q-1}^{v} U^{a, 3}-S_{q^{\prime}-1}^{v} U^{a, 3}\right\|_{L^{2}\left(\mathbb{T}^{3}\right)} & \lesssim 2^{-q}\left\|\left(S_{q-1}^{v}-S_{q^{\prime}-1}^{v}\right) \partial_{3} U^{a, 3}\right\|_{L^{2}\left(\mathbb{T}^{3}\right)}, \\
& \lesssim 2^{-q}\left\|\left(S_{q-1}^{v}-S_{q^{\prime}-1}^{v}\right) \nabla_{h} U_{\mathrm{osc}}\right\|_{L^{2}\left(\mathbb{T}^{3}\right)}, \\
\left\|\triangle_{q}^{\mathrm{v}} \triangle_{q^{\prime}}^{\mathrm{v}} \partial_{3} U_{\mathrm{osc}}\right\|_{H^{1 / 2,0}} & \lesssim 2^{q}\left\|\triangle_{q}^{\mathrm{v}} \triangle_{q^{\prime}}^{\mathrm{v}} U_{\mathrm{osc}}\right\|_{H^{1 / 2,0}},
\end{aligned}
$$

Hence using first 2.4 and then 2.10

$$
C_{2}^{\nu}(q) \lesssim b_{q}(t) 2^{-2 q s}\left\|\nabla_{h} U_{\mathrm{osc}}\right\|_{L^{2}\left(\mathbb{T}^{3}\right)}\left\|U_{\mathrm{osc}}\right\|_{H^{0, s}}\left\|\nabla_{h} U_{\mathrm{osc}}\right\|_{H^{0, s}} .
$$

The term $C_{3}^{\nu}(q)$ will be handled in a different way. First of all, writing $f_{\varepsilon}=\mathcal{L}\left(\frac{t}{\varepsilon}\right) f$ and considering that commutators can be expressed as convolutions (as it has been expressed in detail in the Section 2.3 see equation (2.5)) we can write $C_{3}^{\nu}(q)$ as

$$
\begin{aligned}
C_{3}^{v}(q)=\lim _{\varepsilon \rightarrow 0} \sum_{\left|q-q^{\prime}\right| \leqslant 4} \int_{\mathbb{T}^{3}} \int_{\mathbb{T}_{\nu}^{1} \times[0,1]} \tilde{h}\left(2^{q} y_{3}\right)\left(S_{q^{\prime}-1}^{\mathrm{v}} \partial_{3} U_{\mathrm{osc}, \varepsilon}^{3}\right)\left(x_{h}, x_{3}+\tau\left(x_{3}-y_{3}\right)\right) & \\
& \times \partial_{3} \triangle_{q^{\prime}}^{\mathrm{v}} U_{\mathrm{osc}, \varepsilon}\left(x_{h}, x_{3}-y_{3}\right) \triangle_{q}^{\mathrm{v}} U_{\mathrm{osc}, \varepsilon}(x) \mathrm{d} y_{3} \mathrm{~d} \tau \mathrm{d} x_{h} \mathrm{~d} x_{3},
\end{aligned}
$$

with $\tilde{h}(z)=z h(z)$ and $h=\mathcal{F}^{-1} \varphi$. Taking the limit as $\varepsilon \rightarrow 0$, using the divergence free-property we obtain the following bound

$$
\begin{aligned}
\left|C_{3}^{v}(q)\right| \leqslant \sum_{\left|q-q^{\prime}\right| \leqslant 4} \int_{\mathbb{T}_{v}^{1} \times[0,1]} \sum_{(k, n) \in \mathcal{K}^{\star}} \tilde{h}\left(2^{q} y_{3}\right)\left|\mathcal{F}\left(\left(S_{q^{\prime}-1}^{\mathrm{v}} \nabla_{h} U_{\mathrm{osc}}\right)\left(x_{h}, x_{3}+\tau\left(x_{3}-y_{3}\right)\right)\right)(k)\right| \\
\times\left|\mathcal{F}\left(\partial_{3} \triangle_{q^{\prime}}^{\mathrm{v}} U_{\mathrm{osc}}\left(x_{h}, x_{3}-y_{3}\right)\right)(n-k) \overline{\triangle_{q}^{\mathrm{v}} U_{\mathrm{osc}}}(n)\right| \mathrm{d} y_{3} \mathrm{~d} \tau,
\end{aligned}
$$

applying Lemma 8.4

$$
\begin{aligned}
C_{3}^{v}(q) \lesssim \sum_{\left|q-q^{\prime}\right| \leqslant 4} \int_{\mathbb{T}_{v}^{1} \times[0,1]} \tilde{h}\left(2^{q} y_{3}\right) \| S_{q^{\prime}-1}^{\mathrm{v}} \nabla_{h} U_{\mathrm{osc}}\left(x_{h}, x_{3}+\right. & \left.\tau\left(x_{3}-y_{3}\right)\right) \|_{L^{2}\left(\mathbb{T}^{3}\right)} \\
& \times\left\|\partial_{3} \triangle_{q^{\prime}}^{\mathrm{v}} U_{\mathrm{osc}}\left(x_{h}, x_{3}-y_{3}\right)\right\|_{H^{1 / 2,0}}\left\|\triangle_{q}^{\mathrm{v}} U_{\mathrm{osc}}\right\|_{H^{1 / 2,0}} \mathrm{~d} y_{3} \mathrm{~d} \tau,
\end{aligned}
$$


by standard calculations, localization of the term $\partial_{3} \triangle_{q^{\prime}}^{\mathrm{v}} U_{\mathrm{osc}}$ and 2.7 we obtain

$$
C_{3}^{\nu}(q) \lesssim b_{q}(t) 2^{-2 q s}\left\|\nabla_{h} U_{\mathrm{osc}}\right\|_{L^{2}\left(\mathbb{T}^{3}\right)}\left\|U_{\mathrm{osc}}\right\|_{H^{0, s}}\left\|\nabla_{h} U_{\mathrm{osc}}\right\|_{H^{0, s}}
$$

Lastly, for the reminder term $C_{4}^{\nu}(q)$, if we apply Lemma 8.4 and Lemma 2.1 as for the term $C_{2}^{\nu}(q)$ we get

$$
C_{4}^{v}(q) \lesssim \sum_{q^{\prime}>q-4}\left\|S_{q^{\prime}+2}^{v} U_{\mathrm{osc}}\right\|_{H^{1 / 2,0}}\left\|\triangle_{q^{\prime}}^{v} \nabla_{h} U_{\mathrm{osc}}\right\|_{L^{2}\left(\mathbb{T}^{3}\right)}\left\|\triangle_{q}^{\mathrm{v}} U_{\mathrm{osc}}\right\|_{H^{1 / 2,0}}
$$

hence by localization and the interpolation 2.10 we obtain

$$
C_{4}^{v}(q) \lesssim b_{q}(t) 2^{-2 q s}\left\|U_{\mathrm{osc}}\right\|_{L^{2}\left(\mathbb{T}^{3}\right)}^{1 / 2}\left\|\nabla_{h} U_{\mathrm{osc}}\right\|_{L^{2}\left(\mathbb{T}^{3}\right)}^{1 / 2}\left\|U_{\mathrm{osc}}\right\|_{H^{0, s}}^{1 / 2}\left\|\nabla_{h} U_{\mathrm{osc}}\right\|_{H^{0, s}}^{3 / 2} .
$$

The estimates $8.15-8.20$ prove hence 8.9 .

\subsection{The bilinear form $\mathcal{Q}$}

In this section we state some specific property of the quadratic limit form defined in 3.14. We prove a product rule which can be applied thanks to the particular structure of the resonance set $\mathcal{K}^{\star}=\bigcup_{n \in \mathbb{Z}^{3}} \mathcal{K}_{n}^{\star}$, which is a crucial feature in the energy estimates for the limit system.

The following property has been remarked at first by A. Babin et al. in [6], and it was explicitly proved by M. Paicu in 35. The proof is based on the fact that, fixed $\left(k_{h}, n\right)$, the fiber $\mathcal{J}\left(k_{h}, n\right)=\left\{k_{3}:(k, n) \in \mathcal{K}^{\star}\right\}$ is of finite cardinality.

Lemma 8.4. Let $a, b \in H^{1 / 2,0}\left(\mathbb{T}^{3}\right), c \in L^{2}\left(\mathbb{T}^{3}\right)$ vector fields of zero horizontal average on $\mathbb{T}_{h}^{2}$. Then there exists a constant $C$ which depends on $a_{1} / a_{2}$ only such that

$$
\left|\sum_{(k, n) \in \mathcal{K}^{\star}} \hat{a}(k) \hat{b}(n-k) \hat{c}(n)\right| \leqslant \frac{C}{a_{3}}\|a\|_{H^{1 / 2,0}\left(\mathbb{T}^{3}\right)}\|b\|_{H^{1 / 2,0}\left(\mathbb{T}^{3}\right)}\|c\|_{L^{2}\left(\mathbb{T}^{3}\right)}
$$

The following proof can be found 18 Lemma 6.6, p. 150] or [35. Lemma 6.4, p. 222].

Proof. We prove Lemma 8.4 when $\mathbb{T}^{3}=[0,2 \pi)^{3}$.

$$
\begin{aligned}
I_{\mathcal{K}^{\star}}=\left|\sum_{(k, n) \in \mathcal{K}^{\star}} \hat{a}_{k} \hat{b}_{n-k} \hat{c}_{n}\right| & \leqslant \sum_{\left(k_{h}, n\right) \in \mathbb{Z}^{2} \times \mathbb{Z}^{3}\left\{k_{3}:(k, n) \in \mathcal{K}^{\star}\right\}}\left|\hat{a}_{k} \hat{b}_{n-k} \hat{c}_{n}\right|, \\
& \leqslant \sum_{\left(k_{h}, n\right) \in \mathbb{Z}^{2} \times \mathbb{Z}^{3}}\left|\hat{c}_{n}\right| \sum_{\left\{k_{3}:(k, n) \in \mathcal{K}^{\star}\right\}}\left|\hat{a}_{k}\right|\left|\hat{b}_{n-k}\right| .
\end{aligned}
$$

By Cauchy-Schwarz inequality

$$
\sum_{\left\{k_{3}:(k, n) \in \mathcal{K}^{\star}\right\}}\left|\hat{a}_{k}\right|\left|\hat{b}_{n-k}\right| \leqslant\left(\sum_{\left\{k_{3}:(k, n) \in \mathcal{K}^{\star}\right\}}\left|\hat{a}_{k}\right|^{2}\left|\hat{b}_{n-k}\right|^{2}\right)^{1 / 2}\left(\sum_{\left\{k_{3}:(k, n) \in \mathcal{K}^{\star}\right\}} 1\right)^{1 / 2},
$$

now, fixing $\left(k_{h}, n\right) \in \mathbb{Z}^{2} \times \mathbb{Z}^{3}$ there exists only a finite number (8) of resonant modes $k_{3}$, i.e. \# $\left(\left\{k_{3}:(k, n) \in \mathcal{K}^{\star}\right\}\right) \leqslant 8$. Let us briefly explain why this is true. We write explicitly the resonant condition $\omega_{k, n-k, n}^{+,+,+}=0$ (the same procedure holds for the generic case $\omega_{k, n-k, n}^{a, b, c}=0, a, b, c \neq 0$ ), this reads as

$$
\left(\frac{\left|F k_{3}\right|^{2}+\left|k_{h}\right|^{2}}{\left|k_{3}\right|^{2}+\left|k_{h}\right|^{2}}\right)^{1 / 2}+\left(\frac{\left(F\left|n_{3}-k_{3}\right|\right)^{2}+\left|n_{h}-k_{h}\right|^{2}}{\left|n_{3}-k_{3}\right|^{2}+\left|n_{h}-k_{h}\right|^{2}}\right)^{1 / 2}=\left(\frac{\left|F n_{3}\right|^{2}+\left(\left.n_{h}\right|^{2}\right.}{\left|n_{3}\right|^{2}+\left|n_{h}\right|^{2}}\right)^{1 / 2} .
$$

Taking squares several times on both sides of the above equation give us an expression which is free of square roots. Moreover putting everything to common factor and recalling that $n, k_{h}$ are fixed we transformed the above equation in the form $R\left(k_{3}\right)=0, R \in \mathbb{R}[x]$, hence thanks to fundamental theorem of algebra it has a finite number of roots $k_{3}$.

From this we deduce

$$
\sum_{\left\{k_{3}:(k, n) \in \mathcal{K}^{\star}\right\}}\left|\hat{a}_{k}\right|\left|\hat{b}_{n-k}\right| \leqslant \sqrt{8}\left(\sum_{\left\{k_{3}:(k, n) \in \mathcal{K}^{\star}\right\}}\left|\hat{a}_{k}\right|^{2}\left|\hat{b}_{n-k}\right|^{2}\right)^{1 / 2}
$$

which considered into inequality $[\underline{8.22]}$ gives

$$
I_{\mathcal{K}} \star \leqslant \sqrt{8} \sum_{k_{h}, n_{h}} \sum_{n_{3}}\left|\hat{c}_{n}\right|\left(\sum_{k_{3}}\left|\hat{a}_{k}\right|^{2}\left|\hat{b}_{n-k}\right|^{2}\right)^{1 / 2}
$$


Moreover

$$
\sum_{n_{3}}\left|\hat{c}_{n}\right|\left(\sum_{k_{3}}\left|\hat{a}_{k}\right|^{2}\left|\hat{b}_{n-k}\right|^{2}\right)^{1 / 2} \leqslant\left(\sum_{n_{3}}\left|\hat{c}_{n}\right|^{2}\right)^{1 / 2}\left(\sum_{n_{3}, k_{3}}\left|\hat{a}_{k}\right|^{2}\left|\hat{b}_{n-k}\right|^{2}\right)^{1 / 2}
$$

and hence

$$
I_{\mathcal{K}} \leqslant \sqrt{8} \sum_{\left(k_{h}, n\right) \in \mathbb{Z}^{2} \times \mathbb{Z}^{3}}\left(\sum_{n_{3}}\left|\hat{c}_{n}\right|^{2}\right)^{1 / 2}\left(\sum_{p_{3}}\left|\hat{b}_{n_{h}-k_{h}, p_{3}}\right|^{2}\right)^{1 / 2}\left(\sum_{k_{3}}\left|\hat{a}_{k}\right|^{2}\right)^{1 / 2} .
$$

Let us denote at this point

$$
\tilde{a}_{n_{h}}=\left(\sum_{n_{3}}\left|\hat{a}_{n}\right|^{2}\right)^{1 / 2}, \quad \tilde{b}_{n_{h}}=\left(\sum_{n_{3}}\left|\hat{b}_{n}\right|^{2}\right)^{1 / 2}, \quad \tilde{c}_{n_{h}}=\left(\sum_{n_{3}}\left|\hat{c}_{n}\right|^{2}\right)^{1 / 2},
$$

and the following distributions

$$
\tilde{a}\left(x_{h}\right)=\mathcal{F}_{h}^{-1}\left(\tilde{a}_{n_{h}}\right) \quad \tilde{b}\left(x_{h}\right)=\mathcal{F}_{h}^{-1}\left(\tilde{b}_{n_{h}}\right) \quad \tilde{c}\left(x_{h}\right)=\mathcal{F}_{h}^{-1}\left(\tilde{c}_{n_{h}}\right) .
$$

The inequality 8.23 can be read, applying Plancherel theorem and the product rules for Sobolev spaces, as

$$
\begin{aligned}
I_{\mathcal{K}^{\star}} & \leqslant(\tilde{a} \tilde{b} \mid \tilde{c})_{L^{2}\left(\mathbb{T}_{h}^{2}\right)} \\
& \leqslant\|\tilde{a} \tilde{b}\|_{L^{2}\left(\mathbb{T}_{h}^{2}\right)}\|\tilde{c}\|_{L^{2}\left(\mathbb{T}_{h}^{2}\right)} \\
& \leqslant\|\tilde{a}\|_{H^{1 / 2}\left(\mathbb{T}_{h}^{2}\right)}\|\tilde{b}\|_{H^{1 / 2}\left(\mathbb{T}_{h}^{2}\right)}\|\tilde{c}\|_{L^{2}\left(\mathbb{T}_{h}^{2}\right)} \\
& =\|a\|_{H^{1 / 2,0}\left(\mathbb{T}^{3}\right)}\|b\|_{H^{1 / 2,0}\left(\mathbb{T}^{3}\right)}\|c\|_{L^{2}\left(\mathbb{T}^{3}\right)} .
\end{aligned}
$$

To lift this argument to a generic torus $\prod_{i=1}^{3}\left[0,2 \pi a_{i}\right)$ it suffice to use the transform

$$
\tilde{v}\left(x_{1}, x_{2}, x_{3}\right)=v\left(a_{1} x_{1}, a_{2} x_{2}, a_{3} x_{3}\right) \text {, }
$$

and the identity

$$
\|\tilde{v}\|_{L^{2}\left([0,2 \pi)^{3}\right)}=\left(a_{1} a_{2} a_{3}\right)^{-1 / 2}\|v\|_{L^{2}\left(\Pi_{i=1}^{3}\left[0,2 \pi a_{i}\right)\right)} .
$$

\section{References}

[1] Robert A. Adams and John J. F. Fournier, Sobolev spaces, second ed., Pure and Applied Mathematics (Amsterdam), vol. 140, Elsevier/Academic Press, Amsterdam, 2003.

[2] Serge Alinhac and Patrick Gérard, Opérateurs pseudo-différentiels et théorème de Nash-Moser, Savoirs Actuels. [Current Scholarship], InterEditions, Paris; Éditions du Centre National de la Recherche Scientifique (CNRS), Meudon, 1991.

[3] Jean-Pierre Aubin, Un théorème de compacité, C. R. Acad. Sci. Paris 256 (1963), 5042-5044.

[4] Anatoli Babin, Alex Mahalov, and Basil Nicolaenko, Global splitting, integrability and regularity of $3 D$ Euler and Navier-Stokes equations for uniformly rotating fluids, European J. Mech. B Fluids 15 (1996), no. 3, 291-300.

[5] _ Fast singular oscillating limits and global regularity for the 3D primitive equations of geophysics, M2AN Math. Model. Numer. Anal. 34 (2000), no. 2, 201-222, Special issue for R. Temam's 60th birthday.

[6] __ Global regularity of 3D rotating Navier-Stokes equations for resonant domains, Appl. Math. Lett. 13 (2000), no. 4, 51-57.

[7] Hajer Bahouri, Jean-Yves Chemin, and Raphaël Danchin, Fourier analysis and nonlinear partial differential equations, Grundlehren der Mathematischen Wissenschaften [Fundamental Principles of Mathematical Sciences], vol. 343, Springer, Heidelberg, 2011.

[8] Jean-Michel Bony, Calcul symbolique et propagation des singularités pour les équations aux dérivées partielles non linéaires, Annales scientifiques de l'École Normale Supérieure 14 (1981), no. 2, 209-246 (fre).

[9] Alfred J. Bourgeois and J. Thomas Beale, Validity of the quasigeostrophic model for large-scale flow in the atmosphere and ocean, SIAM J. Math. Anal. 25 (1994), no. 4, 1023-1068.

[10] Frédéric Charve, Global well-posedness and asymptotics for a geophysical fluid system, Comm. Partial Differential Equations 29 (2004), no. 11$12,1919-1940$.

[11] _ Convergence of weak solutions for the primitive system of the quasigeostrophic equations, Asymptot. Anal. 42 (2005), no. 3-4, $173-209$.

[12] _ Estimations de Strichartz pour les équations primitives et convergence quasigéostrophique, Séminaire: Équations aux Dérivées Partielles. 2004-2005, École Polytech., Palaiseau, 2005, pp. Exp. No. XVIII, 17.

[13] Frédéric Charve and Van-Sang Ngo, Global existence for the primitive equations with small anisotropic viscosity, Rev. Mat. Iberoam. 27 (2011), no. 1, 1-38.

[14] Jean-Yves Chemin, À propos d'un problème de pénalisation de type antisymétrique, J. Math. Pures Appl. (9) 76 (1997), no. 9, $739-755$.

[15] _ Perfect incompressible fluids, Oxford Lecture Series in Mathematics and its Applications, vol. 14, The Clarendon Press, Oxford University Press, New York, 1998, Translated from the 1995 French original by Isabelle Gallagher and Dragos Iftimie. 
[16] Jean-Yves Chemin, Benoît Desjardins, Isabelle Gallagher, and Emmanuel Grenier, Fluids with anisotropic viscosity, M2AN Math. Model. Numer. Anal. 34 (2000), no. 2, 315-335, Special issue for R. Temam's 60th birthday.

[17] _ Ekman boundary layers in rotating fluids, ESAIM Control Optim. Calc. Var. 8 (2002), 441-466 (electronic), A tribute to J. L. Lions.

[18] _ Mathematical geophysics, Oxford Lecture Series in Mathematics and its Applications, vol. 32, The Clarendon Press, Oxford University Press, Oxford, 2006, An introduction to rotating fluids and the Navier-Stokes equations.

[19] Jean-Yves Chemin and Nicolas Lerner, Flot de champs de vecteurs non lipschitziens et équations de Navier-Stokes, J. Differential Equations 121 (1995), no. 2, 314-328.

[20] Benoit Cushman-Roisin and Jean-Marie Beckers, Introduction to geophysical fluid dynamics: physical and numerical aspects, vol. 101, Academic Press, 2011.

[21] Alexandre Dutrifoy, Slow convergence to vortex patches in quasigeostrophic balance, Arch. Ration. Mech. Anal. 171 (2004), no. 3, 417-449.

[22] Pedro F. Embid and Andrew J. Majda, Averaging over fast gravity waves for geophysical flows with arbitrary potential vorticity, Comm. Partial Differential Equations 21 (1996), no. 3-4, 619-658.

[23] _ Low Froude number limiting dynamics for stably stratified flow with small or finite Rossby numbers, Geophys. Astrophys. Fluid Dynam. 87 (1998), no. 1-2, 1-50.

[24] Isabelle Gallagher, Applications of Schochet's methods to parabolic equations, J. Math. Pures Appl. (9) 77 (1998), no. 10, $989-1054$.

[25] Isabelle Gallagher and Laure Saint-Raymond, Weak convergence results for inhomogeneous rotating fluid equations, J. Anal. Math. 99 (2006), $1-34$.

[26] Emmanuel Grenier, Oscillatory perturbations of the Navier-Stokes equations, J. Math. Pures Appl. (9) 76 (1997), no. 6, 477-498.

[27] Emmanuel Grenier and Nader Masmoudi, Ekman layers of rotating fluids, the case of well prepared initial data, Comm. Partial Differential Equations 22 (1997), no. 5-6, 953-975.

[28] Dragoş Iftimie, The 3D Navier-Stokes equations seen as a perturbation of the 2D Navier-Stokes equations, Bull. Soc. Math. France 127 (1999), no. $4,473-517$.

[29] _ Approximation of the quasigeostrophic system with the primitive systems, Asymptot. Anal. 21 (1999), no. 2, 89-97.

[30] _ A uniqueness result for the Navier-Stokes equations with vanishing vertical viscosity, SIAM J. Math. Anal. 33 (2002), no. 6, 1483-1493 (electronic). MR 1920641

[31] J.-L. Lions, R. Temam, and S. Wang, New formulations of the primitive equations of atmosphere and applications, Nonlinearity 5 (1992), no. 2, 237-288.

[32] __ Geostrophic asymptotics of the primitive equations of the atmosphere, Topol. Methods Nonlinear Anal. 4 (1994), no. 2, $253-287$.

[33] Fujiwara M, Über die obere schranke des absoluten betrages der wurzeln einer algebraischen gleichung, Tôhoku Math J (1916).

[34] Nader Masmoudi, Ekman layers of rotating fluids: the case of general initial data, Comm. Pure Appl. Math. 53 (2000), no. 4, $432-483$.

[35] Marius Paicu, Étude asymptotique pour les fluides anisotropes en rotation rapide dans le cas périodique, J. Math. Pures Appl. (9) 83 (2004), no. 2, $163-242$.

[36] __ Équation anisotrope de Navier-Stokes dans des espaces critiques, Rev. Mat. Iberoamericana 21 (2005), no. 1, $179-235$.

[37] __ Équation periodique de Navier-Stokes sans viscosité dans une direction, Comm. Partial Differential Equations 30 (2005), no. 7-9, 11071140.

[38] Joseph Pedlosky, Geophysical Fluid Dynamics, Springer-Verlag, 1987.

[39] Steven Schochet, Fast singular limits of hyperbolic PDEs, J. Differential Equations 114 (1994), no. 2, $476-512$.

[40] Elias M. Stein, Harmonic analysis: real-variable methods, orthogonality, and oscillatory integrals, Princeton Mathematical Series, vol. 43, Princeton University Press, Princeton, NJ, 1993, With the assistance of Timothy S. Murphy, Monographs in Harmonic Analysis, III.

BCAM - Basque Center for Applied Mathematics, Mazarredo, 14, E48009 Bilbao, Basque Country - Spain E-mail address: sscrobogna@bcamath.org 